\title{
Reactivity of allyl anions of allylphosphine-boranes towards electrophiles
}

\author{
Marek Stankevič, Marcin Siek, and K. Michał Pietrusiewicz* \\ Department of Organic Chemistry, Faculty of Chemistry, Marie Curie-Sklodowska University, \\ Gliniana 33, 20-614 Lublin, Poland \\ E-mail: kazimierz.pietrusiewicz@poczta.umcs.lublin.pl
}

This paper is dedicated to Prof. William F. Bailey on the occasion of his $65^{\text {th }}$ birthday

DOI: $\underline{\text { http://dx.doi.org/10.3998/ark.5550190.0012.511 }}$

\begin{abstract}
The reactivity of mesomeric carbanions derived from allylphosphine-boranes varies as a function of electrophile. Small $s p^{3}$-electrophiles react predominantly at the $\alpha$ carbon atom whereas bulky $s p^{3}$-electrophiles and carbonyl compounds react mainly at the $\gamma$ carbon. In the case of electrondeficient aldehydes reduction of carbonyl group and formation of the corresponding alcohols is observed. This is attributed to the weakly reducing properties of carbanions derived from allylphosphine-boranes, whose mesomeric form resembles the structure of a modified borohydride.
\end{abstract}

Keywords: Phosphine-boranes, allyl, carbanion, alkylation, reduction

\section{Introduction}

The presence of a multiple carbon-carbon bond in close proximity to phosphorus atom provides a wide the spectrum of reactivity for modifying organophosphorus compounds. Those possessing a $\mathrm{P}$-vinyl substituent can be utilised in Michael addition reactions to provide precursors of $\mathrm{P}-\mathrm{P}^{1}$ or P-N-type ${ }^{2}$ ligands. Ruthenium-catalysed metathesis of vinylphosphine oxides and boranes has been used by several research groups for the synthesis of ethylene-bridged diphosphine derivatives. ${ }^{3}$ The Diels-Alder reaction between vinyl-substituted organophosphorus compounds and P-heterocycles in the presence of an organometallic template can also provide norbornenelike bicyclic diphosphines. ${ }^{4}$ In the case of allyl-substituted organophosphorus compounds, most of the known functionalisation chemistry concerns the $\alpha$-carbanions which are formed upon abstraction of a proton from the allyl moiety by a strong base. When such anions are generated from triphenylphosphonium salts, reaction with carbonyl compounds leads to the formation of a 
conjugated alkene through a Wittig reaction, and its reactivity has been widely explored in the synthesis of natural compounds like callystatin $\mathrm{A},{ }^{5}$ astaxanthin analogues, ${ }^{6}$ ratjadone, ${ }^{7}$ epolactaene, ${ }^{8}$ viridenomycin ${ }^{9}$ or superstolide A. ${ }^{10}$ In such cases, however, the loss of the phosphorus moiety is necessary to complete the synthesis. In the cases where the phosphorus moiety is retained in the final product, the mesomeric nature of phosphorus-substituted allyl anion means that the formation of two isomeric compounds is to be expected (Scheme 1).
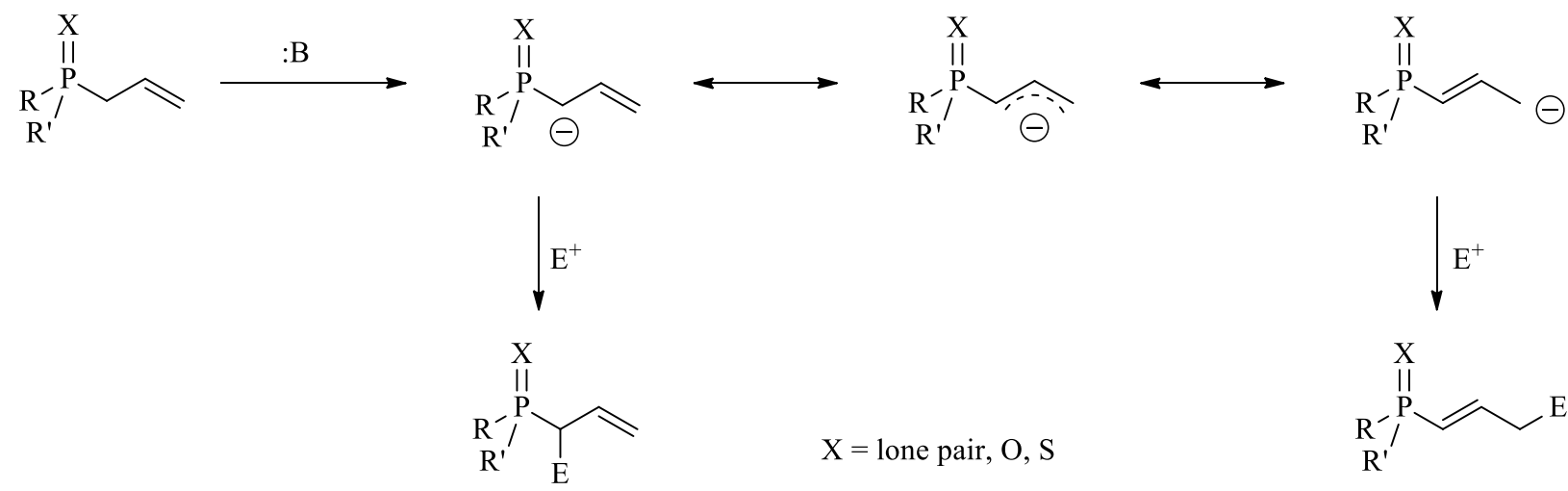

Scheme 1. Mesomeric forms of allyl anion derived from P-allyl compounds.

The outcome of such a reaction is influenced by the nature of the substrates employed and the reaction conditions, with less sterically demanding electrophiles generally preferring the $\alpha$-position, whilst more sterically encumbered electrophiles favour the $\gamma$-position. Results obtained so far for allylphosphonates show that $s p^{3}$-electrophiles and aldehydes react preferentially at the $\alpha$-position ${ }^{11}$ whereas ketones react at both the $\alpha$ - and the $\gamma$-carbon. ${ }^{12}$ On the other hand, $\alpha, \beta$-unsaturated carbonyls react mainly at the $\gamma$-carbon atom of the allyl moiety. ${ }^{13}$ Surprisingly little is known about the reactivity of other classes of allyl-substituted organophosphorus compounds, ${ }^{14}$ including phosphine-boranes, which are very attractive substitutes for free phosphines because they are devoid of many typical shortcomings of free phosphines such as sensitivity to oxygen and moisture. These features explain why phosphineboranes are often used in the synthesis of phosphine ligands for transition metal-catalysed reactions. ${ }^{15}$ Surprisilgly, the only known application of phosphine-borane-derived allyl carbanions can be found in one of our recent studies ${ }^{16}$ which demonstrated that the alkylation of a 3-phospholene-borane with dihaloalkanes in the presence of an excess of base can lead to the formation of a bicyclic phosphine-borane as the major reaction product (Scheme 2)
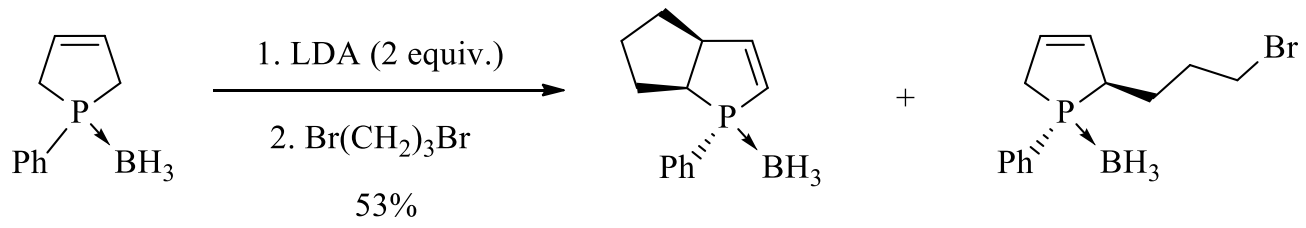

traces 
Scheme 2. Cyclopentannulation of 1-phenylphosphol-3-ene-borane.

The usefulness and generality of these allyl anion-induced annulations was further confirmed by the synthesis of $P, C$-chiral bicyclic monophosphine ligands which showed enantioselectivities up to $95 \%$ ee in palladium-catalysed allylic substitution reactions. ${ }^{17}$

The structural complexity of the bicyclic phosphine-borane formed from simple allylphosphine-boranes nicely illustrates the ambivalent reactivity of phosphorus-substituted allyl anions and suggests that, if controlled appropriately, this could be used in the synthesis of a wide range of useful compounds. To achieve this, a basic understanding of the reactivity of such systems is needed. Here, we present our results concerning the reactivity of allyl carbanions derived from allyphosphine-boranes or phosphol-3-ene-boranes.

\section{Results and Discussion}

Three model phosphine-boranes: allyl-t-butylphenylphosphine-borane 1a, allyldiphenylphosphine-borane $\mathbf{1 b}$ and 1-phenylphosphol-3-ene-borane 1c (Chart 1), were chosen for test reactions because they present different structural motifs having diaryl, arylalkyl and arylcycloalkyl substitution patterns at phosphorus.

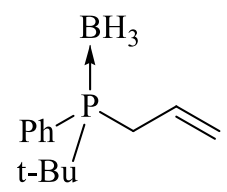

$1 \mathbf{a}$

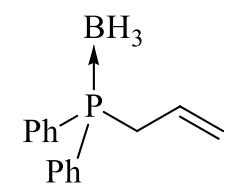

$1 \mathbf{b}$

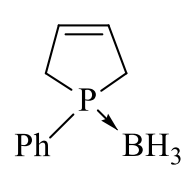

$1 \mathbf{c}$

Chart 1. Model phosphine-boranes.

Allyl-t-butylphenylphosphine-borane 1a was synthesized in three steps from phenyldichlorophosphine as shown in Scheme 3.

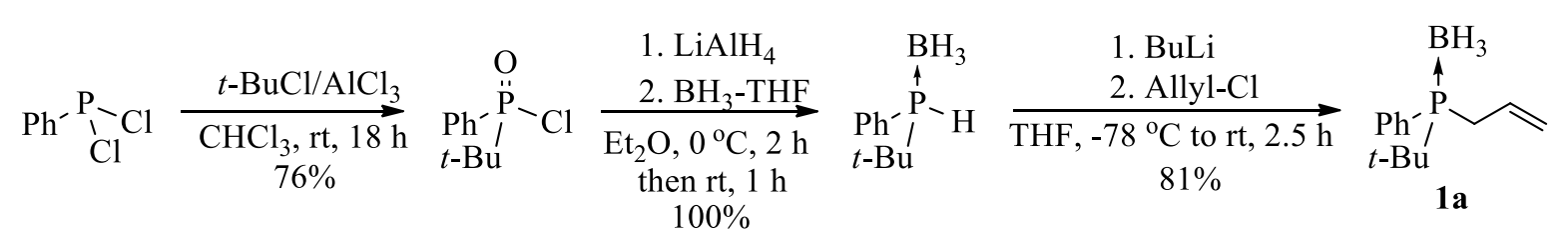

Scheme 3. The synthesis of allyl(t-butyl)phenylphosphine-borane 1a.

Treatment of $\mathrm{PhPCl}_{2}$ with $t$-butyl chloride in the presence of $\mathrm{AlCl}_{3}$ followed by hydrolysis gave $t$-butylphenylphosphinic chloride. Subsequent reduction with $\mathrm{LiAlH}_{4}$ and in situ trapping 
with $\mathrm{BH}_{3}$ afforded the corresponding secondary phosphine-borane, which was in turn allylated in the presence of butyllithium.

Allyldiphenylphosphine-borane $\mathbf{1 b}$ was prepared in three steps starting from commercially available triphenylphosphine (Scheme 4).

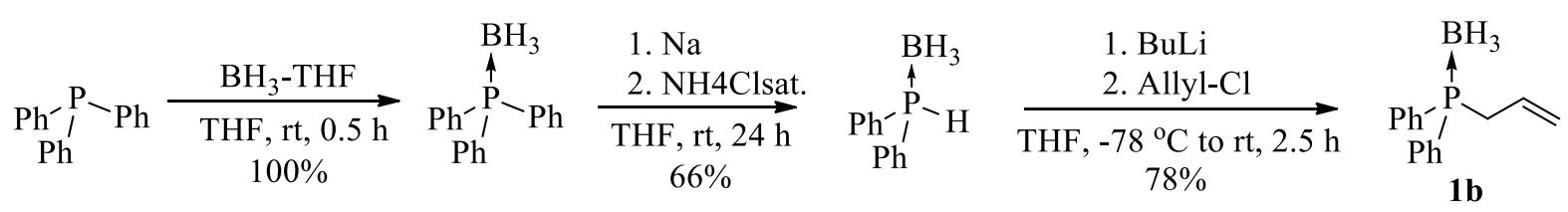

Scheme 4. The synthesis of allyldiphenylphosphine-borane $\mathbf{1 b .}$

Triphenylphosphine was treated with the $\mathrm{BH}_{3}$-THF complex to give the corresponding phosphine-borane quantitatively. Removal of one phenyl group from the phosphorus atom was accomplished by treatment with sodium and the resulting secondary phosphine-borane was converted to allylphosphine-borane by allylation with BuLi/Allyl-Cl.

1-Phenylphosphol-3-ene-borane 1c was prepared from the corresponding phosphine oxide in two steps ${ }^{16 \mathrm{~b}}$ through reduction of $\mathrm{P}=\mathrm{O}$ bond with $\mathrm{LiAlH}_{4}$ and in situ complexation of tertiary phosphine with $\mathrm{BH}_{3}$-THF.

With these three model compounds in hands, we started our studies of the alkylation of Pallyl carbanions with a range of alkyl halides (Table 1).<smiles>[R][P]([13CH3])([13CH3])c1ccccc1</smiles>
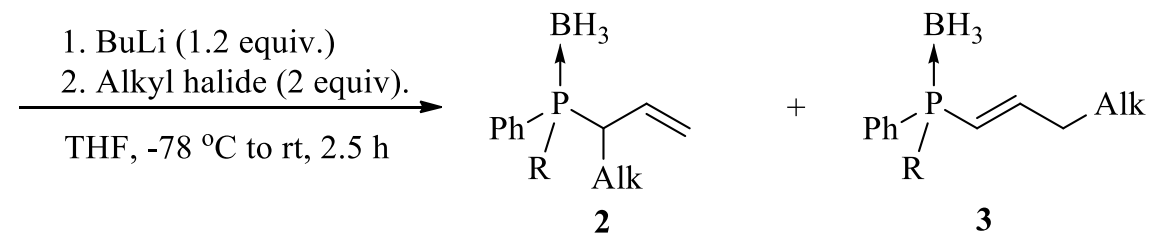

Table 1. Reactivity of 1a-b towards alkylating agents

\begin{tabular}{|c|c|c|c|c|c|c|}
\hline Entry & Electrophile & Phosphine- & \multicolumn{4}{|c|}{ Products $(\%)$} \\
\hline 1 & \multirow{2}{*}{$\operatorname{MeI}(\mathbf{a})$} & $\mathbf{1 a}$ & 2aa & 68 & 3aa & 15 \\
\hline 2 & & $1 b$ & $2 \mathbf{b a}$ & 88 & & \\
\hline 3 & \multirow{2}{*}{$\mathrm{BnCl}(\mathbf{b})$} & $\mathbf{1 a}$ & 2ab & $29 *$ & 3ab & 21 \\
\hline 4 & & 1b & $2 b b$ & 76 & 3bb & 13 \\
\hline 5 & \multirow{2}{*}{ AllylCl (c) } & $\mathbf{1 a}$ & $2 a c$ & 13 & 3ac & 62 \\
\hline 6 & & $1 b$ & $2 b c$ & 42 & 3bc & 42 \\
\hline 7 & \multirow{2}{*}{ c-HexBr $(\mathbf{d})$} & $\mathbf{1 a}$ & & & 3ad & 47 \\
\hline 8 & & 1b & 2bd & 22 & 3bd & 47 \\
\hline 9 & $\operatorname{TMSCl}(\mathbf{e})$ & $1 \mathbf{a}$ & & & 3ae & 91 \\
\hline 10 & $\mathrm{BrCH}_{2} \mathrm{COOEt}(\mathbf{f})$ & $1 \mathbf{a}$ & & & 3af & 20 \\
\hline 11 & $\mathrm{BrCH}_{2} \mathrm{COOEt}(\mathbf{f})$ & 1a & 2af & $23 * *$ & & \\
\hline
\end{tabular}


* - when performed in $\mathrm{Et}_{2} \mathrm{O}$, the reaction afforded $\mathbf{2 a b}$ as the sole product in $58 \%$ yield. ** - the reaction was performed at $0{ }^{\circ} \mathrm{C}$.

It can be seen from Table 1 that only methyl iodide, the smallest electrophile, gave completely regioselective alkylation at the $\alpha$-carbon of the P-allyl carboanion (entries 1 and 2, Table 1). Other electrophiles react with phosphine-boranes $\mathbf{1 a}$ and $\mathbf{1 b}$ at both the $\alpha$ - and $\gamma$ carbanionic sites. The course of the reaction seems to be governed by the steric hindrance at the reaction centre; this control is more pronounced for 1a which possesses a bulky tert-butyl group attached to the phosphorus atom than for $\mathbf{1 b}$ which features a smaller phenyl substituent. Steric crowding at the phosphorus atom impedes the approach of the electrophile at the $\alpha$-carbon atom thus favouring its reaction through the $\gamma$-carbocation. This is clearly seen in the reactions of $\mathbf{1 a}$ with different electrophiles (entries 1,3,5,7,9), where replacement of methyl iodide with the more bulky benzyl chloride gives less clean $\alpha$-alkylation and no $\alpha$-alkylation at all is found when cyclohexyl bromide or trimethylsilyl chloride are used as electrophiles. An interesting effect was observed in the reaction of $\mathbf{1 a}$ with ethyl bromoacetate. When performed at $-78{ }^{\circ} \mathrm{C}$, the reaction afforded the $\gamma$-alkylation product 3af in $20 \%$ yield (Table 1, entry 10) whilst changing the temperature to $0{ }^{\circ} \mathrm{C}$ gave only $\alpha$-alkylation product 2af in 23\% yield (Table 1, entry 11).

Being sterically less demanding than a $t$-butyl substituent, the phenyl group gives higher yields of $\alpha$-alkylation products (entries 2,4,6,8, Table 1). Even with cyclohexyl bromide the formation of $\alpha$-alkylation product was still observed at a reasonable level.

Unlike 1a and 1b, phosphine-borane 1c gave only $\alpha$-alkylation products, regardless of the electrophile used. Another striking observation for 1c was the formation of bis- $\alpha, \alpha$-alkylated products in the reaction with benzyl and allyl chlorides (Scheme 5).
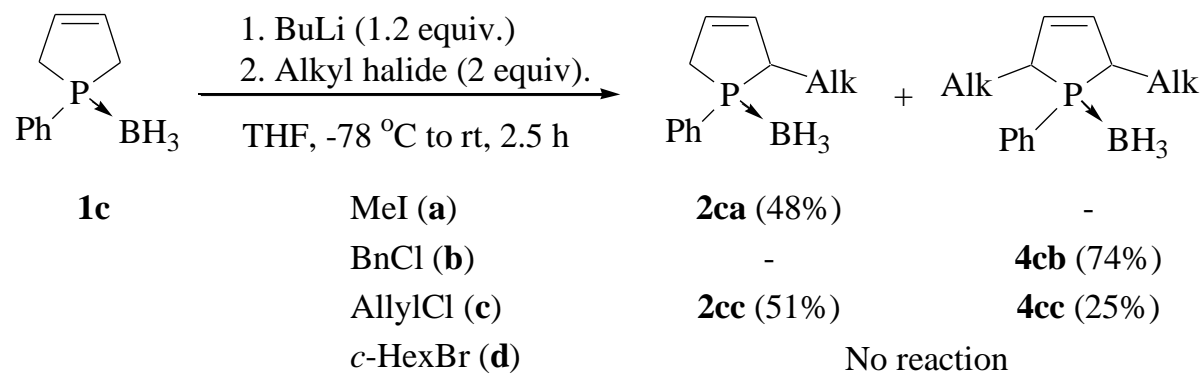

Scheme 5. Reaction of $1 \mathbf{c}$ with various alkyl halides.

The same type of behaviour has previously been observed by Pakulski et al. in the reactions of 3-phospholene oxides and sulphides with alkylating agents (Scheme 2). ${ }^{16}$ Another potentially useful application of $\mathrm{P}$-allyl anions is their homocoupling, which provides diphosphines having two double bonds in the carbon skeleton linking the two phosphorus atoms. Given the two mesomeric forms of phosphorus-substituted allyl anion, three isomeric $\alpha, \alpha-, \gamma, \gamma-$ and $\alpha, \gamma-$ coupling products 5, $\mathbf{6}$ or 7 can theoretically be formed (Scheme 6). Each of them appears to have a potentially useful unsaturation pattern: activated double bonds for Michael addition or 
Diels-Alder reaction in $\mathbf{5}$, or non-activated allylic double bonds for radical reactions or polymerisation in $\mathbf{6}$ and 7. In the event, treatment of the allyl anion generated from phosphineborane $1 \mathrm{a}$ and $\mathrm{LDA}$ with $\mathrm{CuCl}_{2}$ or $\mathrm{FeCl}_{3}$ led to the formation of only the $\gamma, \gamma$-coupled product in $20 \%$ and $66 \%$ yields, respectively (Scheme 6).
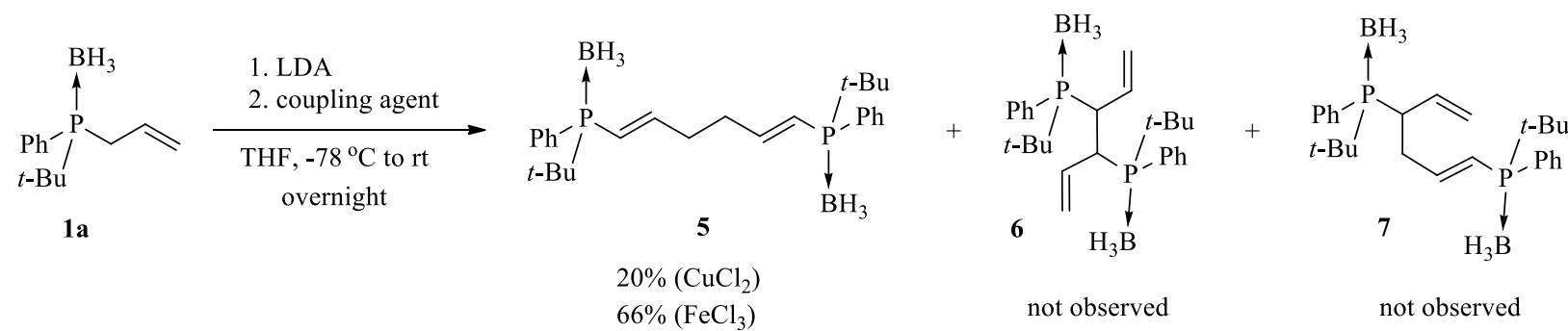

not observed

not observed

Scheme 6. Homocoupling of $1 \mathbf{a}$.

We next turned our attention to $s p^{2}$-type electrophiles and explored their reactivity towards the P-allyl anions derived from 1a-c (Table 2). The results collected in the Table 2 clearly show that, under basic conditions, phosphine-boranes 1a,b react as $\gamma$-carbon nucleophiles towards carbonyl compounds to give the corresponding $\delta$-hydroxy- $\alpha$-alkenylphosphine-boranes 8 exclusively, in good to excellent yields. Formaldehyde however, failed to give clean conversion and instead yielded a complex product mixtures. With acetophenone as the electrophile (Table 2, entry 11), the allyl anion of 1a afforded a mixture of two $\gamma$-addition products: 8al and 9al, the latter possibly arising from isomerisation of the double bond in 8al. Another striking observation was the reaction of methyl vinyl ketone $\mathbf{m}$ with the allyl anions of $\mathbf{1 a}$ and $\mathbf{1 b}$ (Table 2, entries 13 , 14). In this case, the allyl anions reacted exclusively through 1,2-addition to form the corresponding $\gamma$-alcohols in good yields. This stands in sharp contrast to previous observations of the reactivity of phosphoryl-substituted allyl anions with $\alpha, \beta$-unsaturated carbonyl compounds where exclusively 1,4-addition products were observed in the reaction mixture. ${ }^{13}$ Reactions with methyl vinyl ketone also revealed a sensitivity to steric demands. In the case of allyl-tbutylphenylphosphine-borane 1a, the only product isolated from the reaction mixture resulted from $\gamma$-addition, whereas in the case of $\mathbf{1 b}$ substantial quantities of $\alpha$-addition product were observed (Table 2, entry 14). With ethyl acetate as the electrophile, the only compound isolated from the reaction mixture was the tertiary alcohol 8an, an evolution product which results from the addition of butyllithium to the carbonyl group of the first-formed $\gamma$-acyl product (Table 2 , entry 15).

In the case of borane 1a, addition of the allyl anion to carbonyl compound possessing a prochiral $\mathrm{C}=\mathrm{O}$ group created additional chirality at the centre $\delta$ to the phosphorus atom. However, as there was no steric differentiation in the proximity of the $\gamma$ carbon atom, the reaction of 1a with prochiral carbonyl reagents prodeeded with low diatereoselectivity in all cases (Table 2, entries 1, 3, 5, 11 and 13). 
Addition of carbon dioxide to the allyl anion of $\mathbf{1 a}, \mathbf{b}$ yielded the corresponding (4boranatophosphinyl)but-3-enoic acids in 76 and 92\% yields, respectively (Table 2, entries 16 and 17).
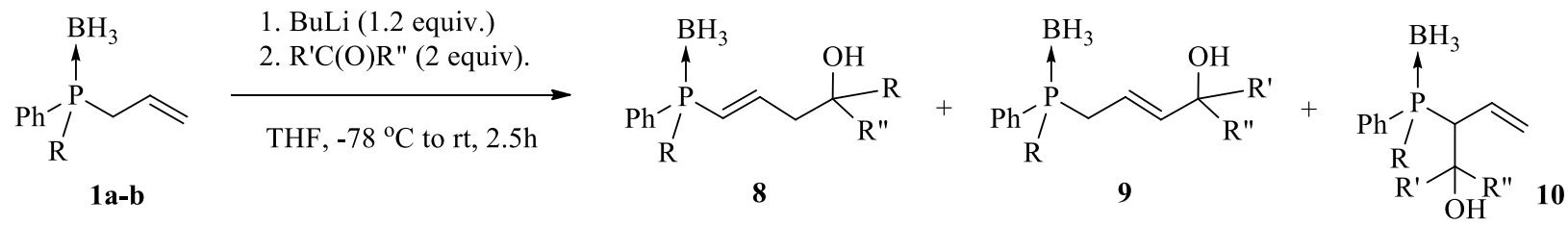

Table 2. Reactivity of 1a-b towards carbonyl compounds

\begin{tabular}{ccccc}
\hline Entry & Electrophile & $\begin{array}{c}\text { Phosphine- } \\
\text { borane }\end{array}$ & \multicolumn{2}{c}{ Products (\%) } \\
\hline 1 & benzaldehyde $(\mathbf{g})$ & $\mathbf{1 a}$ & $\mathbf{8 a g}$ & $97(61: 39$ d.r. $)$ \\
2 & & $\mathbf{1 b}$ & $\mathbf{8 b g}$ & 77 \\
3 & p-tolulaldehyde $(\mathbf{h})$ & $\mathbf{1 a}$ & $\mathbf{8 a h}$ & $64(50: 50$ d.r. $)$ \\
4 & & $\mathbf{1 b}$ & $\mathbf{8 b h}$ & 65 \\
5 & o-tolulaldehyde $(\mathbf{i})$ & $\mathbf{1 a}$ & $\mathbf{8 a i}$ & $67(53: 47$ d.r. $)$ \\
6 & & $\mathbf{1 b}$ & $\mathbf{8 b i}$ & 73 \\
7 & acetone $(\mathbf{j})$ & $\mathbf{1 a}$ & $\mathbf{8 a j}$ & 99 \\
8 & & $\mathbf{1 b}$ & $\mathbf{8 b j}$ & 73 \\
9 & cyclohexanone $(\mathbf{k})$ & $\mathbf{1 a}$ & $\mathbf{8 a k}$ & 71 \\
10 & & $\mathbf{1 b}$ & $\mathbf{8 b k}$ & 53 \\
11 & acetophenone $(\mathbf{l})$ & $\mathbf{1 a}$ & $\mathbf{8 a l}$ & $36(56: 44$ d.r. $)$ \\
12 & & $\mathbf{1 b}$ & $\mathbf{9 a l}$ & $24(62: 38$ d.r. $)$ \\
13 & methyl vinyl ketone $(\mathbf{m})$ & $\mathbf{1 a}$ & $\mathbf{8 b l}$ & 67 \\
14 & & $\mathbf{1 b}$ & $\mathbf{8 a m}$ & $84(58: 42$ d.r. $)$ \\
15 & ethyl acetate $(\mathbf{n})$ & $\mathbf{1 a}$ & $\mathbf{8 b m : 1 0 b m}$ & $91(45: 55)$ \\
16 & & $\mathbf{1 a}$ & $\mathbf{8 a n}$ & 81 \\
17 & carbon dioxide $(\mathbf{o})$ & $\mathbf{1 b}$ & $\mathbf{8 a 0}$ & 76 \\
& & & $\mathbf{8 b o}$ & 92 \\
\hline
\end{tabular}

Unlike phosphine-boranes 1a and 1b, the cyclic phosphine-borane 1c reacted only as an $\alpha$-nucleophiles but isomerisation of the double bond was observed in some cases. This may reflect strongly basic conditions present in the reaction mixture (Scheme 7). 


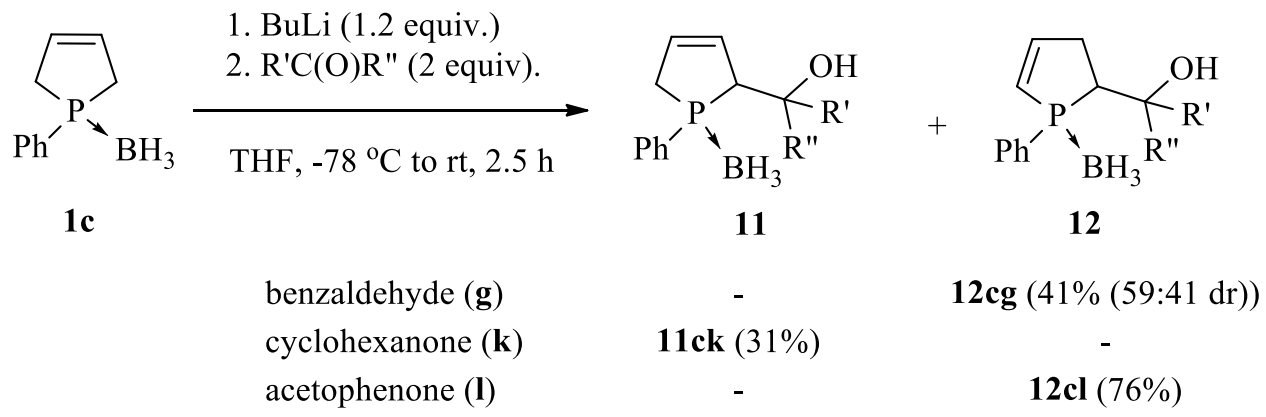

Scheme 7. Reactivity of 1c towards carbonyl compounds.

Interesting results were obtained in the reaction between P-allyl anions derived from 1a and 1b and methyl chloroformate (Scheme 8).

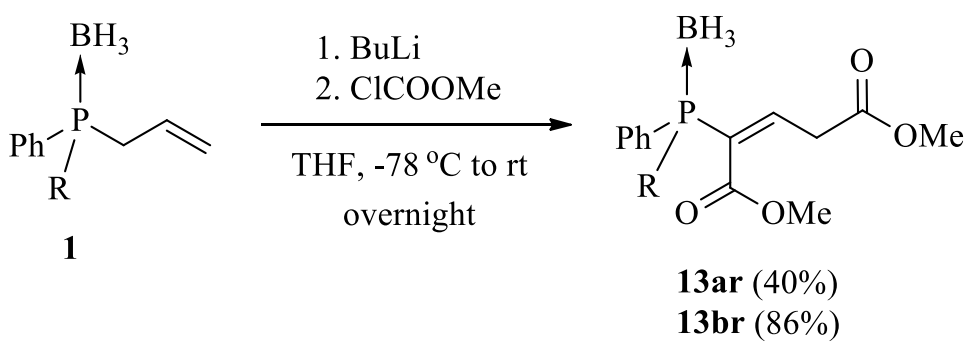

Scheme 8. Reaction of 1a with methyl chloroformate.

In both cases, the formation of $\alpha, \gamma$-disubstitution products was observed, in $40 \%$ and $86 \%$ yields for $\mathbf{1 a}$ and for $\mathbf{1 b}$, respectively. It seems likely, that the formation of these unusual products can be explained in terms of an enhanced reactivity of the intermediate monosubstitution product with respect to the parent P-allyl anion.

An interesting feature of the reactions of allylphosphine-borane anions with some electron deficient carbonyl electrophiles was the reduction of the carbonyl compounds (Scheme 9).

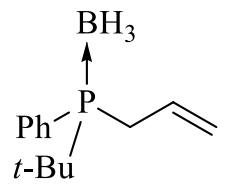

1a

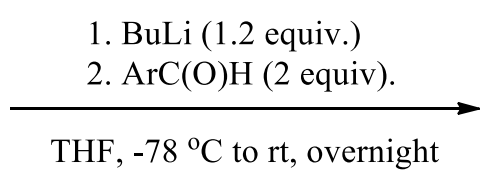

$$
\mathrm{Ar}=\quad o-\mathrm{NO}_{2}-\mathrm{C}_{6} \mathrm{H}_{4}(\mathbf{q})
$$<smiles>OC([Al])CC=CP(Br)(Br)(CBr)CBr</smiles>

8ap $(18 \%)^{*}$ 8aq $(36 \%)^{*}$<smiles>O=[N+]([O-])c1ccc(CO)cc1</smiles>

$14 p(26 \%)^{*}$ $14 \mathbf{q}(29 \%) *$

* - yields calculated from ${ }^{1} \mathrm{H}$ NMR spectra

Scheme 9. Reaction of 1a with nitro-substituted benzaldehydes under basic conditions. 
In the reaction of 1a with $p$-nitrobenzaldehyde the yield of the expected adduct 8ap was only $18 \%\left({ }^{1} \mathrm{H}\right.$ NMR) while the yield of $p$-nitrobenzyl alcohol 12p was $26 \%$ based on consumed aldehyde. When $o$-nitrobenzaldehyde was used as the reactant, the yield of expected 8aq was estimated to be $36 \%$ ( ${ }^{1} \mathrm{H}$ NMR), whereas the yield of $o$-nitrobenzyl alcohol $\mathbf{1 2 q}$ was $29 \%$ as based on aldehyde consumption. The formation of such benzyl alcohols in reactions, where no reducing agent was added to the reaction mixture, points to the allylphosphine-borane anions as potential reducing agents.

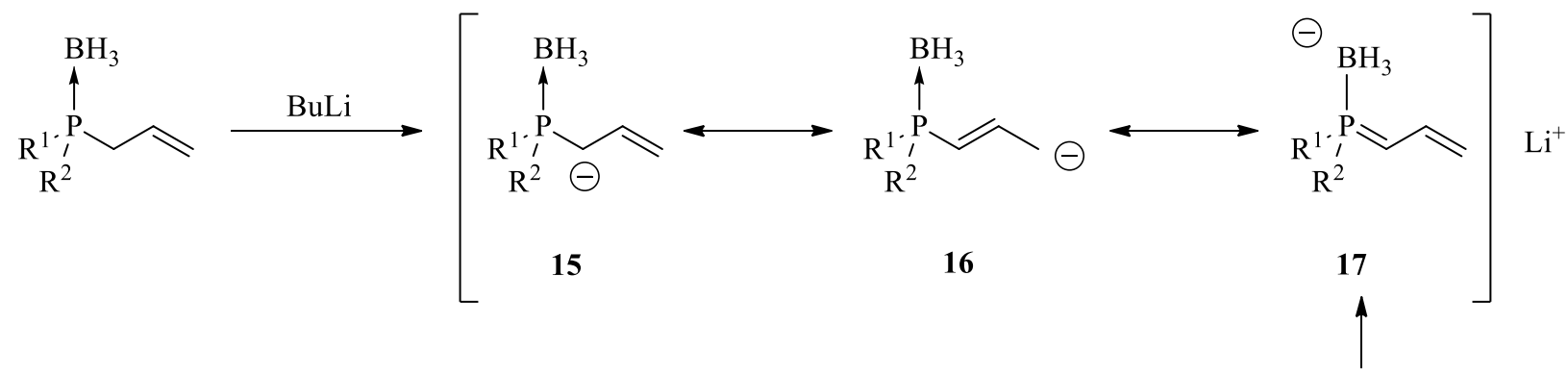

a modified lithium borohydride

Scheme 10. Formulation of an allylphosphine-borane anion as amodified lithium borohydride.

As depicted in Scheme 10, deprotonation of allylphosphine-borane leads to the formation of an anion whose structure can be drawn in three mesomeric forms 15-17. Form 17 localises some anionic electron density at boron and can be regarded as a modified borohydride anion. Borohydrides are known to reduce carbonyl groups in aldehydes and ketones easily but is apparently less reactive and exhibits its reducing properties only towards electron deficient aldehydes (Scheme 11).

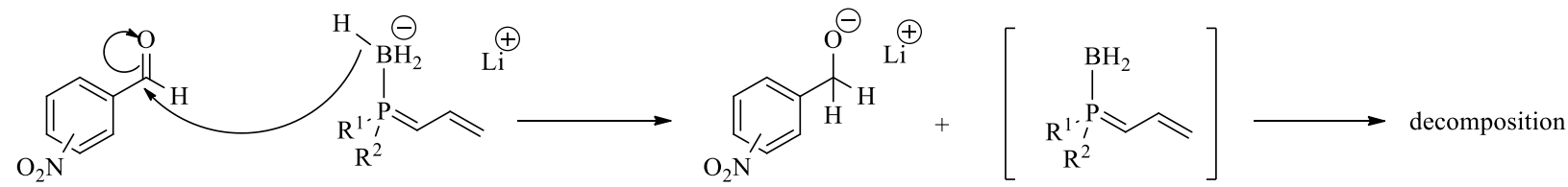

Scheme 11. Plausible mechanism for the reduction of carbonyl groups by a modified borohydride.

\section{Conclusions}

In conclusion, the reactivity of mesomeric allylphosphine-borane-derived anions derived from allylphosphine-boranes towards electrophiles depends strongly on the structure of the starting allylphosphine-borane and on the electrophile. Small $s p^{3}$-electrophiles react predominantly at the $\alpha$-carbon atom, whereas sterically demanding $s p^{3}$-electrophiles react mostly at the $\gamma$-carbon. On 
the other hand, $s p^{2}$-electrophiles react with such allyl anions at $\gamma$-carbon atom to give $\delta$-hydroxy$\alpha$-alkenylphosphine-boranes in good yields, except in the case of electron deficient carbonyl compounds which are reduced by the phosphine-borane anions to the corresponding alcohols instead of being attacked by the allyl anion. Homodimerisation of allylphosphine-borane-derived anions provides 1,6-diphosphine-diboranes possessing a 1,5-hexadienyl linker.

\section{Experimental Section}

General. All reactions were conducted under inert atmosphere and with anhydrous and oxygenfree solvents. THF was dried under sodium/benzophenone, diethyl ether was dried under metallic sodium and chloroform was dried under $\mathrm{P}_{2} \mathrm{O}_{5}$. All electrophiles used in reactions were commercially available and were used as received. The course of reaction was followed by TLC (Merck, silica $60 \mathrm{~F}_{254}$, aluminium plates) and iodine/ $\mathrm{SiO}_{2}$ was used as the developing agent. Flash chromatography was performed using silica gel (Merck, 20-230 mesh) with hexane:ethyl acetate 2-50:1 as eluents. NMR analyses were performed on Bruker Avance 300 in $\mathrm{CDCl}_{3}$ with TMS as internal standard and elementary analysis was performed on PERKIN ELMER CHN 2400 at the Department of Organic Chemistry, Marie Curie-Skłodowska University, Lublin, Poland.

\section{Synthesis of allyl-t-butylphenylphosphine-borane (1a)}

t-Butylphenylphosphinic chloride. In a two-necked flask $(500 \mathrm{~mL})$ equipped with magnetic stirrer, argon inlet and dropping funnel was placed $200 \mathrm{~mL}$ of chloroform, $23 \mathrm{~mL}(0.17 \mathrm{~mol})$ dichlorophosphine, and $25 \mathrm{~g}(0.188 \mathrm{~mol})$ of $\mathrm{AlCl}_{3}$ and the mixture was stirred at room temperature for $30 \mathrm{~min}$ until all $\mathrm{AlCl}_{3}$ dissolved. Then, the flask was immerced in the ice-water bath and $25 \mathrm{~mL}(0.226 \mathrm{~mol})$ of $t$-butyl chloride was slowly added via dropping funnel. After complete addition of $t$ - $\mathrm{BuCl}$ the reaction mixture was stirred at room temperature for overnight. Then, diluted hydrochloric acid $(10 \%, 150 \mathrm{~mL})$ was added and mixture was extracted with DCM $(5 \times 100 \mathrm{~mL})$. Combined organic phases were dried over magnesium sulfate, filtered and evaporated and the residue was distilled under low pressure yielding $t$-butylphenylphosphinic chloride as colorless oil which solidified upon standing in refrigerator $(24.3 \mathrm{~g}, 66 \%)$. b.p. 156 ${ }^{\circ} \mathrm{C} / 15 \mathrm{~mm} \mathrm{Hg} .{ }^{1} \mathrm{H} \mathrm{NMR}\left(\mathrm{CDCl}_{3}, 300 \mathrm{MHz}\right): \delta 1.26$ (d, $\left.J_{\mathrm{P}-\mathrm{H}} 19.1 \mathrm{~Hz}, t-\mathrm{Bu}, 9 \mathrm{H}\right), 7.45-7.70$ (m, Ph, 3H), 7.79-7.96 (m, Ph, 2H); ${ }^{13} \mathrm{C} \mathrm{NMR}\left(\mathrm{CDCl}_{3}, 75 \mathrm{MHz}\right): \delta 24.12,38.93\left(\mathrm{~d}, J_{\mathrm{P}-\mathrm{C}} 77.4 \mathrm{~Hz}\right), 128.25$ $\left(\mathrm{d}, J_{\mathrm{P}-\mathrm{C}} 12.8 \mathrm{~Hz}\right), 132.45\left(\mathrm{~d}, J_{\mathrm{P}-\mathrm{C}} 10.0 \mathrm{~Hz}\right), 132.81$ (d, $\left.J_{\mathrm{P}-\mathrm{C}} 2.9 \mathrm{~Hz}\right) .{ }^{31} \mathrm{P} \mathrm{NMR}\left(\mathrm{CDCl}_{3}, 121.5\right.$ $\mathrm{MHz}$ ): $\delta 74.3$ ppm. Elem. anal. for $\mathrm{C}_{10} \mathrm{H}_{14} \mathrm{ClOP}$ : calc. C 55.44, H 6.51; found C 55.59, H 6.82.

$\boldsymbol{t}$-Butylphenylphosphine-borane. In a $500 \mathrm{~mL}$ two-necked flask equipped with magnetic stirrer, ice-water bath and argon inlet was placed $150 \mathrm{~mL}$ of diethyl ether and $2.40 \mathrm{~g}(11 \mathrm{mmol})$ of $t$ butylphenylphosphinic chloride. Reaction mixture was cooled to $0{ }^{\circ} \mathrm{C}$ and $0.84 \mathrm{~g}(22 \mathrm{mmol})$ of lithium aluminum hydride was added in one portion and reaction was stirred at $0{ }^{\circ} \mathrm{C}$ for two 
hours. Then, excess of $\mathrm{LiAlH}_{4}$ was removed by slow addition of water until no bubbling was observed. The formed solids were removed by filtration through $2 \mathrm{~cm}$ layer of silica gel under argon and the solid was washed with DCM $(100 \mathrm{~mL})$. The solution was then placed in the twonecked flask equipped with magnetic stirrer and argon inlet and $22 \mathrm{~mL}(22 \mathrm{mmol})$ of $1 \mathrm{M} \mathrm{BH}_{3}-$ THF complex was added. Reaction was stirred at room temperature for an hour and then was evaporated and the residue was dissolved in $50 \mathrm{~mL}$ of DCM and filtered. Evaporation yielded $1.98 \mathrm{~g}(100 \%)$ of $t$-butylphenylphosphine-borane as colorless oil. ${ }^{1} \mathrm{H} \mathrm{NMR}\left(\mathrm{CDCl}_{3}, 300 \mathrm{MHz}\right): \delta$ -0.05-1.65 (bm, BH $3,3 \mathrm{H}$ ); 1.21 (d, $J_{\mathrm{P}-\mathrm{H}} 14.8 \mathrm{~Hz}, t$-Bu, 9H), 5.13 (dq, $J_{\mathrm{P}-\mathrm{H}} 368.0 \mathrm{~Hz}, 1 \mathrm{H}, \mathrm{P}-\mathrm{H}$ ); 7.41-7.73 (m, 5H, Ph). ${ }^{13} \mathrm{C} \mathrm{NMR}\left(\mathrm{CDCl}_{3}, 75 \mathrm{MHz}\right): \delta 26.54\left(\mathrm{~d}, J_{\mathrm{P}-\mathrm{C}} 2.9 \mathrm{~Hz}\right), 28.46\left(\mathrm{~d}, J_{\mathrm{P}-\mathrm{C}} 32.5\right.$ $\mathrm{Hz}), 124.74\left(\mathrm{~d}, J_{\mathrm{P}-\mathrm{C}} 51.0 \mathrm{~Hz}\right), 128.54$ (d, $\left.J_{\mathrm{P}-\mathrm{C}} 9.7 \mathrm{~Hz}\right) .131 .50\left(\mathrm{~d}, J_{\mathrm{P}-\mathrm{C}} 3.5 \mathrm{~Hz}\right), 133.88$ (d, $J_{\mathrm{P}-\mathrm{C}} 7.7$ $\mathrm{Hz}) .{ }^{31} \mathrm{P} \mathrm{NMR}\left(\mathrm{CDCl}_{3}, 121.5 \mathrm{MHz}\right)$ : $\delta 31.99 \mathrm{ppm}(\mathrm{m})$. Elem. anal. for $\mathrm{C}_{10} \mathrm{H}_{15} \mathrm{BP}$ : calc. C 66.83, $\mathrm{H}$ 10.21; found C 67.15, H 10.48 .

Allyl-t-butylphenylphosphine-borane (1a). In a $250 \mathrm{~mL}$ two-necked flask equipped with magnetic stirrer, dry ice-acetone bath, and argon inlet was placed $100 \mathrm{~mL}$ of THF and $1.98 \mathrm{~g}$ (11 mmol) of $t$-butylphenylphosphine-borane. Then, $10 \mathrm{~mL}(16.5 \mathrm{mmol})$ of $1.6 \mathrm{M}$ butyllithium was added and mixture was stirred at $-78{ }^{\circ} \mathrm{C}$ for 30 minutes. Then, $1.8 \mathrm{~mL}$ (22 mmol) of allyl chloride was added and reaction mixture was left at room temperature for overnight. Then, 10 $\mathrm{mL}$ of $5 \% \mathrm{HCl}$ was added with subsequent $30 \mathrm{~mL}$ of distilled water and the mixture was extracted with DCM $(3 \times 50 \mathrm{~mL})$. Organic phases were dried over magnesium sulfate, filtered and evaporated. The residue was purified by flash chromatography using hexane/EtOAc 6:1 as eluent yielding $2.0 \mathrm{~g}(83 \%)$ of allyl-t-butylphenylphosphine-borane $\mathbf{1 a}$ as pasty white solid. ${ }^{1} \mathrm{H}$ NMR $\left(\mathrm{CDCl}_{3}, 300 \mathrm{MHz}\right): \delta$ 0.13-1.29 (bm, BH $\left.3,3 \mathrm{H}\right), 1.12$ (d, $\left.J_{\mathrm{P}-\mathrm{H}} 13.7 \mathrm{~Hz}, t-\mathrm{Bu}, 9 \mathrm{H}\right), 2.72-2.86(\mathrm{~m}$, $1 \mathrm{H}), 2.88-3.04(\mathrm{~m}, 1 \mathrm{H}), 5.05-5.21(\mathrm{~m}, 2 \mathrm{H}), 7.40-7.53(\mathrm{~m}, 3 \mathrm{H}), 7.66-7.75(\mathrm{~m}, 2 \mathrm{H}) .{ }^{13} \mathrm{C} \mathrm{NMR}$ $\left(\mathrm{CDCl}_{3}, 75 \mathrm{MHz}\right): \delta 24.74\left(\mathrm{~d}, J_{\mathrm{P}-\mathrm{C}} 33.1 \mathrm{~Hz}\right), 25.04\left(\mathrm{~d}, J_{\mathrm{P}-\mathrm{C}} 2.3 \mathrm{~Hz}\right), 28.89$ (d, $\left.J_{\mathrm{P}-\mathrm{C}} 30.8 \mathrm{~Hz}\right)$, $119.18\left(\mathrm{~d}, J_{\mathrm{P}-\mathrm{C}} 10.6 \mathrm{~Hz}\right), 125.19\left(\mathrm{~d}, J_{\mathrm{P}-\mathrm{C}} 48.3 \mathrm{~Hz}\right), 127.76\left(\mathrm{~d}, J_{\mathrm{P}-\mathrm{C}} 9.2 \mathrm{~Hz}\right), 128.76\left(\mathrm{~d}, J_{\mathrm{P}-\mathrm{C}} 4.6 \mathrm{~Hz}\right)$, $130.73\left(\mathrm{~d}, J_{\mathrm{P}-\mathrm{C}} 2.3 \mathrm{~Hz}\right), 133.16\left(\mathrm{~d}, J_{\mathrm{P}-\mathrm{C}} 7.6 \mathrm{~Hz}\right) .{ }^{31} \mathrm{P} \mathrm{NMR}\left(\mathrm{CDCl}_{3}, 121.5 \mathrm{MHz}\right): \delta 30.36(\mathrm{~m})$. Elem. anal. for $\mathrm{C}_{13} \mathrm{H}_{19} \mathrm{BP}$ : calc. C 70.93, H 10.09; found C 70.18, H 10.01.

\section{Synthesis of allyldiphenylphosphine-borane (1b)}

Diphenylphosphine-borane. In a $250 \mathrm{~mL}$ two-necked flask equipped with magnetic stirrer and argon inlet was placed $100 \mathrm{~mL}$ of THF, $10 \mathrm{~g}(38 \mathrm{mmol})$ of triphenylphosphine and $38 \mathrm{~mL}$ (38 mmol) of $1 \mathrm{M} \mathrm{BH}_{3}$-THF was added. After one hour the mixture was evaporated yielding pure triphenylphosphine-borane which was used directly in the next step.

In $250 \mathrm{~mL}$ two-necked flask equipped with magnetic stirrer and argon inlet was placed $100 \mathrm{~mL}$ of THF, $5.0 \mathrm{~g}$ (18 mmol) of triphenylphosphine-borane and $0.83 \mathrm{~g}(36 \mathrm{mmol})$ of metallic sodium was added. After stirring for $24 \mathrm{~h}$ at $\mathrm{rt}$ reaction was finished by adding saturated ammonium chloride solution $(50 \mathrm{~mL})$. The formed mixture was extracted with DCM $(3 \times 100 \mathrm{~mL})$ dried over anhydrous magnesium sulfate, filtered and evaporated. The residue was purified on silica gel using hexane/EtOAc 6:1 as eluent yielding $5.02 \mathrm{~g}(66 \%)$ of diphenylphosphine-borane as colorless oil. ${ }^{1} \mathrm{H} \mathrm{NMR}\left(\mathrm{CDCl}_{3}, 300 \mathrm{MHz}\right): \delta$ 0.25-1.95 (bm, $\left.\mathrm{BH}_{3}, 3 \mathrm{H}\right), 6.35$ (dq, $J_{\mathrm{P}-\mathrm{H}} 380.0 \mathrm{~Hz}$, 
1H), 7.41-7.79 (m, 10H). ${ }^{13} \mathrm{C} \mathrm{NMR}\left(\mathrm{CDCl}_{3}, 75 \mathrm{MHz}\right): \delta 126,11\left(\mathrm{~d}, J_{\mathrm{P}-\mathrm{C}} 56.9 \mathrm{~Hz}\right), 128.98\left(\mathrm{~d}, J_{\mathrm{P}-\mathrm{C}}\right.$ $10.6 \mathrm{~Hz}$ ), 131.56 (d, $\left.J_{\mathrm{P}-\mathrm{C}} 2.4 \mathrm{~Hz}\right) .132 .85$ (d, $\left.J_{\mathrm{P}-\mathrm{C}} 9.3 \mathrm{~Hz}, \mathrm{P}-\mathrm{Ph}\right) .{ }^{31} \mathrm{P}$ NMR $\left(\mathrm{CDCl}_{3}, 121.5 \mathrm{MHz}\right): \mathrm{d}$ 2.89 (m). Elem. anal. for $\mathrm{C}_{12} \mathrm{H}_{14} \mathrm{BP}$ : calc. C 71.93, H 6.90; found C 72.15, H 7.09.

Preparation of allyldiphenylphosphine-borane (1b). In a Schlenk flask equipped with magnetic stirrer and argon inlet was placed diphenylphosphine-borane $(2.34 \mathrm{~g}, 11.7 \mathrm{mmol})$ in 80 $\mathrm{mL}$ of dry THF. Then, reaction was cooled to $-78{ }^{\circ} \mathrm{C}$ and $1.6 \mathrm{M} \mathrm{BuLi}(11 \mathrm{~mL}, 17.6 \mathrm{mmol})$ was added and the mixture was stirred at $-78{ }^{\circ} \mathrm{C}$ for $0.5 \mathrm{~h}$. Then, the bath was taken out and allyl chloride $(1.91 \mathrm{~mL}, 22 \mathrm{mmol})$ was added and the mixture was allowed to stir for overnight. Then, reaction mixture was quenched with saturated ammonium chloride solution and extracted three times with DCM $(3 \times 30 \mathrm{~mL})$. Combined organic phases were dried over anhydrous magnesium sulfate, filtered and evaporated to dryness. The residue was purified by flash chromatography using hexane/EtOAc 6:1 as eluent yielding allyldiphenylphosphine-borane $\mathbf{1 b}(2.20 \mathrm{~g}, 78 \%)$ as colorless oil. ${ }^{1} \mathrm{H}$ NMR $\left(\mathrm{CDCl}_{3}, 300 \mathrm{MHz}\right): \delta$ 0.30-1.40 (bm, $\left.\mathrm{BH}_{3}, 3 \mathrm{H}\right) ; 3.04-3.13(\mathrm{~m}, 2 \mathrm{H}), 5.03-$ $5.11(\mathrm{~m}, 2 \mathrm{H}), 5.72-5.89(\mathrm{~m}, 1 \mathrm{H}), 7.42-7.55(\mathrm{~m}, 6 \mathrm{H}), 7.65-7.74(\mathrm{~m} 4 \mathrm{H}) .{ }^{13} \mathrm{C} \mathrm{NMR}\left(\mathrm{CDCl}_{3}, 75\right.$ MHz): 26.18 (d, $\left.J_{\mathrm{P}-\mathrm{C}} 40.2 \mathrm{~Hz}\right), 120.02$ (d, $\left.J_{\mathrm{P}-\mathrm{C}} 11.5 \mathrm{~Hz}\right), 128.18$ (d, $\left.J_{\mathrm{P}-\mathrm{C}} 9.8 \mathrm{~Hz}\right), 128.99\left(\mathrm{~d}, J_{\mathrm{P}-\mathrm{C}}\right.$ $5.5 \mathrm{~Hz}), 129.36\left(\mathrm{~d}, J_{\mathrm{P}-\mathrm{C}} 54.6 \mathrm{~Hz}\right), 130.74\left(\mathrm{~d}, J_{\mathrm{P}-\mathrm{C}} 2.6 \mathrm{~Hz}\right), 133.05\left(\mathrm{~d}, J_{\mathrm{P}-\mathrm{C}} 8.3 \mathrm{~Hz}\right) .{ }^{31} \mathrm{P} \mathrm{NMR}$ $\left(\mathrm{CDCl}_{3}, 121.5 \mathrm{MHz}\right): \delta 15.88(\mathrm{~m})$. Elem. anal. for $\mathrm{C}_{15} \mathrm{H}_{18} \mathrm{BP}$ : calc. C 75.04, $\mathrm{H}$ 7.56; found $\mathrm{C}$ $74.80, \mathrm{H} 7.27$.

\section{General procedure for reaction between allylphosphine-boranes 1 and $s p^{3}$ electrophiles}

In a Schlenk flask equipped with magnetic stirrer and argon inlet was placed allylphosphineborane $1(0.19-0.76 \mathrm{mmol})$ in $10 \mathrm{~mL}$ of dry THF. Then, reaction was cooled to $-78{ }^{\circ} \mathrm{C}$ and $\mathrm{BuLi}$ (1.6 $\mathrm{M}$ in hexanes) $(0.28-1.142 \mathrm{mmol})$ was added and the resulting mixture was stirred at $-78^{\circ} \mathrm{C}$ temperature for $0.5 \mathrm{~h}$. Then, an electrophile $(0.38-1.52 \mathrm{mmol})$ was added and the reaction mixture was allowed to warm to room temperature overnight. Then, the reaction was quenched with saturated $\mathrm{NH}_{4} \mathrm{Cl}$ solution and extracted with DCM $(3 \times 15 \mathrm{~mL})$. Combined organic phases were dried over anhydrous magnesium sulfate, filtered and evaporated to dryness. The residue was purified by flash chromatography using hexane/EtOAc 2:1 to 50:1 as eluent to give product. (But-1-en-3-yl)-t-butylphenylphosphine-borane (2aa). Yield 68\% from $0.076 \mathrm{~g}(0.35 \mathrm{mmol})$ of 1, purified with hexane/EtOAc (10/1), white solid, m.p. 42-44 ${ }^{\circ} \mathrm{C} .{ }^{1} \mathrm{H} \mathrm{NMR}\left(\mathrm{CDCl}_{3}, 300\right.$ MHz): $\delta$ 0.02-1.11 (bm, BH $3,3 \mathrm{H}), 0.97\left(\mathrm{~d}, J_{\mathrm{P}-\mathrm{H}} 7.0 \mathrm{~Hz}, 3 \mathrm{H}, \mathrm{CH}_{3}\right), 1.04\left(\mathrm{~d}, J_{\mathrm{P}-\mathrm{H}} 13.5 \mathrm{~Hz}, 9 \mathrm{H}, t\right.$ $\mathrm{Bu}), 3.17-3.35(\mathrm{~m}, 1 \mathrm{H}), 5.07-5.14(\mathrm{~m}, 1 \mathrm{H}), 5.17-5.27(\mathrm{~m}, 1 \mathrm{H}), 5.99-6.20(\mathrm{~m}, 1 \mathrm{H}), 7.30-7.45(\mathrm{~m}$, $3 \mathrm{H}), 7.62-7.73(\mathrm{~m}, 2 \mathrm{H}) .{ }^{13} \mathrm{C} \mathrm{NMR}\left(\mathrm{CDCl}_{3}, 75 \mathrm{MHz}\right): \delta 17.22,26.15\left(\mathrm{~d}, J_{\mathrm{P}-\mathrm{C}} 2.0 \mathrm{~Hz}\right), 30.36\left(\mathrm{~d}, J_{\mathrm{P}-}\right.$ C $30.2 \mathrm{~Hz}), 32.39$ (d, $\left.J_{\mathrm{P}-\mathrm{C}} 31.9 \mathrm{~Hz}\right), 116.70$ (d, $\left.J_{\mathrm{P}-\mathrm{C}} 10.4 \mathrm{~Hz}\right), 126.90$ (d, $\left.J_{\mathrm{P}-\mathrm{C}} 48.0 \mathrm{~Hz}\right), 128.26$ (d, $\left.J_{\mathrm{P}-\mathrm{C}} 8.9 \mathrm{~Hz}\right), 130.97\left(\mathrm{~d}, J_{\mathrm{P}-\mathrm{C}} 2.3 \mathrm{~Hz}\right), 133.23\left(\mathrm{~d}, J_{\mathrm{P}-\mathrm{C}} 7.2 \mathrm{~Hz}\right), 138.01 .{ }^{31} \mathrm{P} \mathrm{NMR}\left(\mathrm{CDCl}_{3}, 121.5\right.$ $\mathrm{MHz}): \delta 37.80(\mathrm{~m})$. Elem. anal. for $\mathrm{C}_{14} \mathrm{H}_{24} \mathrm{BP}$ : calc. C 71.82, $\mathrm{H} 10.33$; found $\mathrm{C} 71.99, \mathrm{H} 10.50$.

(But-1-en-1-yl)-t-butylphenylphosphine-borane (3aa). Yield 15\% from $0.076 \mathrm{~g}(0.35 \mathrm{mmol})$ of 1a, purified with hexane/EtOAc (10/1), colorless oil. ${ }^{1} \mathrm{H}$ NMR $\left(\mathrm{CDCl}_{3}, 300 \mathrm{MHz}\right): \delta$ 0.10$1.17\left(\mathrm{bm}, \mathrm{BH}_{3}, 3 \mathrm{H}\right), 1.08\left(\mathrm{~d}, J_{\mathrm{P}-\mathrm{H}} 13.7 \mathrm{~Hz}, 9 \mathrm{H}, t-\mathrm{Bu}\right), 1.09$ (t, $\left.J_{\mathrm{H}-\mathrm{H}} 6.9 \mathrm{~Hz}, 1 \mathrm{H}\right), 2.25-2.35(\mathrm{~m}$, 2H), 6.16-6.26 (m, 1H), 6.78-7.00 (m, 1H), 7.43-7.53 (m, 3H), 7.69-7.76 (m, 2H). ${ }^{13} \mathrm{C}$ NMR 
$\left(\mathrm{CDCl}_{3}, 75 \mathrm{MHz}\right): \delta 12.28\left(\mathrm{~d}, J_{\mathrm{P}-\mathrm{C}} 1.2 \mathrm{~Hz}\right), 25.33\left(\mathrm{~d}, J_{\mathrm{P}-\mathrm{C}} 2.9 \mathrm{~Hz}\right), 27.96\left(\mathrm{~d}, J_{\mathrm{P}-\mathrm{C}} 36.5 \mathrm{~Hz}\right), 35.16$ $\left(\mathrm{d}, J_{\mathrm{P}-\mathrm{C}} 14.7 \mathrm{~Hz}\right), 112.47\left(\mathrm{~d}, J_{\mathrm{P}-\mathrm{C}} 56.0 \mathrm{~Hz}\right), 128.08\left(\mathrm{~d}, J_{\mathrm{P}-\mathrm{C}} 9.5 \mathrm{~Hz}\right), 130.71$ (d, $\left.J_{\mathrm{P}-\mathrm{C}} 2.6 \mathrm{~Hz}\right)$, $133.00\left(\mathrm{~d}, J_{\mathrm{P}-\mathrm{C}} 7.8 \mathrm{~Hz}\right), 156.32\left(\mathrm{~d}, J_{\mathrm{P}-\mathrm{C}} 9.2 \mathrm{~Hz}\right) .{ }^{31} \mathrm{P} \mathrm{NMR}\left(\mathrm{CDCl}_{3}, 121.5 \mathrm{MHz}\right): \delta 24.83(\mathrm{~m})$. Elem. anal. for $\mathrm{C}_{14} \mathrm{H}_{24} \mathrm{BP}$ : calc. C 71.82, $\mathrm{H}$ 10.33; found $\mathrm{C} 71.78, \mathrm{H} 10.61$.

(But-1-en-3-yl)-diphenylphosphine-borane (2ba). Yield $88 \%$ from $0.077 \mathrm{~g}(0.32 \mathrm{mmol})$ of $\mathbf{1 b}$, purified with hexane/EtOAc (6/1), colorless oil. ${ }^{1} \mathrm{H} \mathrm{NMR}\left(\mathrm{CDCl}_{3}, 300 \mathrm{MHz}\right)$ : $\delta$ 0.24-1.32 (bm, $\left.\mathrm{BH}_{3}, 3 \mathrm{H}\right), 1.18$ (dd, $\left.J_{\mathrm{H}-\mathrm{H}} 7.0 \mathrm{~Hz}, J_{\mathrm{P}-\mathrm{H}} 16.28 \mathrm{~Hz}, \mathrm{CH}_{3}, 3 \mathrm{H}\right), 3.13-3.32(\mathrm{~m}, 1 \mathrm{H}), 4.84-5.01$ (m, 2H),

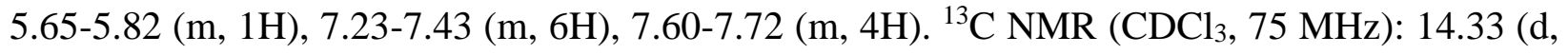
$\left.J_{\mathrm{P}-\mathrm{C}} 1.5 \mathrm{~Hz}\right), 34.89\left(\mathrm{~d}, J_{\mathrm{P}-\mathrm{C}} 34.5 \mathrm{~Hz}\right), 117.76\left(\mathrm{~d}, J_{\mathrm{P}-\mathrm{C}} 10.1 \mathrm{~Hz}\right), 127.90\left(\mathrm{~d}, J_{\mathrm{P}-\mathrm{C}} 54.0 \mathrm{~Hz}\right), 128.26(\mathrm{~d}$, $\left.J_{\mathrm{P}-\mathrm{C}} 50.9 \mathrm{~Hz}\right), 128.42\left(\mathrm{~d}, J_{\mathrm{P}-\mathrm{C}} 9.8 \mathrm{~Hz}\right), 128.70$ (d, $\left.J_{\mathrm{P}-\mathrm{C}} 10.1 \mathrm{~Hz}\right), 131.10$ (d, $\left.J_{\mathrm{P}-\mathrm{C}} 2.3 \mathrm{~Hz}\right), 131.12(\mathrm{~d}$, $\left.J_{\mathrm{P}-\mathrm{C}} 2.0 \mathrm{~Hz}\right), 132.58\left(\mathrm{~d}, J_{\mathrm{P}-\mathrm{C}} 8.3 \mathrm{~Hz}\right), 133.00\left(\mathrm{~d}, J_{\mathrm{P}-\mathrm{C}} 8.3 \mathrm{~Hz}\right), 135.15\left(\mathrm{~d}, J_{\mathrm{P}-\mathrm{C}} 2.9 \mathrm{~Hz}\right) .{ }^{31} \mathrm{P}$ NMR $\left(\mathrm{CDCl}_{3}, 121.5 \mathrm{MHz}\right): \delta 23.29(\mathrm{~m})$. Elem. anal. for $\mathrm{C}_{16} \mathrm{H}_{20} \mathrm{BP}$ : calc. C 75.62, H 7.93; found C $75.80, \mathrm{H} 7.95$.

2-Methyl-1-phenyl-3-phospholene-borane (2ca). Yield $48 \%$ from $0.050 \mathrm{~g}(0.28 \mathrm{mmol})$ of 1c, purified with hexane/EtOAc (6/1), colorless oil. ${ }^{1} \mathrm{H} \mathrm{NMR}\left(\mathrm{CDCl}_{3}, 300 \mathrm{MHz}\right): \delta$ 0.13-1.32 (bm, $\left.\mathrm{BH}_{3}, 3 \mathrm{H}\right), 1.27$ (dd, $\left.J_{\mathrm{P}-\mathrm{H}} 16.7 \mathrm{~Hz}, J_{\mathrm{P}-\mathrm{H}} 7.3 \mathrm{~Hz}, \mathrm{CH}_{3}, 3 \mathrm{H}\right), 2.61-2.88$ (m, 2H), 2.90-3.03 (m, 1H), 5.79-5.92 (m, 2H), 7.31-7.46 (m, 3H), 7.61-7.70 (m, 2H). ${ }^{13} \mathrm{C} \mathrm{NMR}\left(\mathrm{CDCl}_{3}, 75 \mathrm{MHz}\right): \delta 16.04$ $\left(\mathrm{d}, J_{\mathrm{P}-\mathrm{C}} 4.9 \mathrm{~Hz}\right), 32.86\left(\mathrm{~d}, J_{\mathrm{P}-\mathrm{C}} 35.6 \mathrm{~Hz}\right), 39.62\left(\mathrm{~d}, J_{\mathrm{P}-\mathrm{C}} 34.5 \mathrm{~Hz}\right), 126.73\left(\mathrm{~d}, J_{\mathrm{P}-\mathrm{C}} 0.9 \mathrm{~Hz}\right), 128.77$ $\left(\mathrm{d}, J_{\mathrm{P}-\mathrm{C}} 9.8 \mathrm{~Hz}\right), 131.10\left(\mathrm{~d}, J_{\mathrm{P}-\mathrm{C}} 8.6 \mathrm{~Hz}\right), 131.35$ (d, $\left.J_{\mathrm{P}-\mathrm{C}} 2.6 \mathrm{~Hz}\right), 131.38$ (d, $\left.J_{\mathrm{P}-\mathrm{C}} 52.3 \mathrm{~Hz}\right), 135.98$ $\left(\mathrm{d}, J_{\mathrm{P}-\mathrm{C}} 4.9 \mathrm{~Hz}\right) .{ }^{31} \mathrm{P}$ NMR $\left(\mathrm{CDCl}_{3}, 121.5 \mathrm{MHz}\right): \delta 32.20(\mathrm{~m})$. Elem. anal. for $\mathrm{C}_{11} \mathrm{H}_{16} \mathrm{BP}$ : calc. C 69.52, H 8.49; found C 69.40, H 8.40.

t-Butylphenyl-(4-phenylbut-1-en-3-yl)phosphine-borane (2ab). Yield 29\% from $0.051 \mathrm{~g}(0.23$ $\mathrm{mmol})$ of 1a, purified with hexane/EtOAc (10/1), colorless oil. ${ }^{1} \mathrm{H} \mathrm{NMR}\left(\mathrm{CDCl}_{3}, 300 \mathrm{MHz}\right): \delta$ 0.11-1.28 (bm, BH $3,3 \mathrm{H}), 1.06$ (d, $J_{\mathrm{P}-\mathrm{H}} 13.6 \mathrm{~Hz}, t$-Bu, 9H), 2.44-2.66 (m, 2H), 3.15-3.30 (m, 1H), 4.74-4.83 (m, 1H), 5.03-5.09 (m, 1H), 5.91-6.07 (m, 1H), 6.85-6.91 (m, 2H), 7.04-7.19 (m, 3H), 7.41-7.50 (m, 3H), 7.77-7.85 (m, 2H). ${ }^{13} \mathrm{C} \mathrm{NMR}\left(\mathrm{CDCl}_{3}, 75 \mathrm{MHz}\right): \delta 26.20\left(\mathrm{~d}, J_{\mathrm{P}-\mathrm{C}} 1.7 \mathrm{~Hz}\right)$, 30.69 (d, JP-C $29.6 \mathrm{~Hz}), 36.23$ (d, $\left.J_{\mathrm{P}-\mathrm{C}} 2.3 \mathrm{~Hz}\right), 40.95$ (d, J $\left.J_{\mathrm{P}-\mathrm{C}} 30.8 \mathrm{~Hz}\right), 119.51$ (d, $\left.J_{\mathrm{P}-\mathrm{C}} 10.9 \mathrm{~Hz}\right)$, 126.19, 126.98 (d, JP-C $47.7 \mathrm{~Hz}), 128.02,128.54$ (d, $\left.J_{\mathrm{P}-\mathrm{C}} 9.2 \mathrm{~Hz}\right), 129.20,131.29$ (d, $\left.J_{\mathrm{P}-\mathrm{C}} 2.3 \mathrm{~Hz}\right)$, $133.33\left(\mathrm{~d}, J_{\mathrm{P}-\mathrm{C}} 7.2 \mathrm{~Hz}\right), 134.79,139.12\left(\mathrm{~d}, J_{\mathrm{P}-\mathrm{C}} 12.6 \mathrm{~Hz}\right) .{ }^{31} \mathrm{P} \mathrm{NMR}\left(\mathrm{CDCl}_{3}, 121.5 \mathrm{MHz}\right): \delta 36.96$ (m). Elem. anal. for $\mathrm{C}_{20} \mathrm{H}_{28}$ BP: calc. C 77.43, H 9.10; found C 77.70, H 9.31.

t-Butylphenyl-(4-phenylbut-1-en-1-yl)phosphine-borane (3ab). Yield 21\% from $0.051 \mathrm{~g}(0.23$ mmol) of 1a, purified with hexane/EtOAc (10/1), colorless oil. ${ }^{1} \mathrm{H}$ NMR $\left(\mathrm{CDCl}_{3}, 300 \mathrm{MHz}\right): \delta$ 0.07-1.28 (bm, BH $3,3 \mathrm{H}), 0.95\left(\mathrm{~d}, J_{\mathrm{P}-\mathrm{H}} 13.9 \mathrm{~Hz}, t-\mathrm{Bu}, 9 \mathrm{H}\right), 2.51-2.61(\mathrm{~m}, 2 \mathrm{H}), 2.71-2.79(\mathrm{~m}, 2 \mathrm{H})$, 6.01-6.12 (m, 1H), 6.71-6.89 (m, 1H), 7.04-7.15 (m, 3H), 7.15-7.24 (m, 2H), 7.28-7.40 (m, 3H), 7.51-7.60 (m, 2H). ${ }^{13} \mathrm{C} \mathrm{NMR}\left(\mathrm{CDCl}_{3}, 75 \mathrm{MHz}\right): \delta 25.31$ (d, J $\left.J_{\mathrm{P}-\mathrm{C}} 2.6 \mathrm{~Hz}\right), 29.45\left(\mathrm{~d}, J_{\mathrm{P}-\mathrm{C}} 35.4 \mathrm{~Hz}\right)$, $34.29\left(\mathrm{~d}, J_{\mathrm{P}-\mathrm{C}} 1.4 \mathrm{~Hz}\right), 36.75\left(\mathrm{~d}, J_{\mathrm{P}-\mathrm{C}} 14.7 \mathrm{~Hz}\right), 114.82\left(\mathrm{~d}, J_{\mathrm{P}-\mathrm{C}} 54.9 \mathrm{~Hz}\right), 126.04,128.11$ (d, $J_{\mathrm{P}-\mathrm{C}} 9.5$ $\mathrm{Hz}), 128.43,128.45,128.90\left(\mathrm{~d}, J_{\mathrm{P}-\mathrm{C}} 43.7 \mathrm{~Hz}\right), 130.73$ (d, $\left.J_{\mathrm{P}-\mathrm{C}} 2.6 \mathrm{~Hz}\right), 133.03$ (d, $\left.J_{\mathrm{P}-\mathrm{C}} 8.3 \mathrm{~Hz}\right)$, 140.71, 153.58 (d, $\left.J_{\mathrm{P}-\mathrm{C}} 9.5 \mathrm{~Hz}\right) .{ }^{31} \mathrm{P} \mathrm{NMR}\left(\mathrm{CDCl}_{3}, 121.5 \mathrm{MHz}\right): \delta 25,28 \mathrm{ppm}(\mathrm{m})$. Elem. anal. for $\mathrm{C}_{20} \mathrm{H}_{28} \mathrm{BP}$ : calc. C 77.43, H 9.10; found C 77.25, H 9.00. 
Diphenyl-(4-phenylbut-1-en-3-yl)phosphine-borane (2bb). Yield 76\% from $0.081 \mathrm{~g}(0.33$ mmol) of 1b, purified with hexane/EtOAc (50/1), colorless oil. ${ }^{1} \mathrm{H}$ NMR $\left(\mathrm{CDCl}_{3}, 300 \mathrm{MHz}\right)$ : $\delta$ 0.33-1.63 (bm, $\left.\mathrm{BH}_{3}, 3 \mathrm{H}\right), 2.66-2.92(\mathrm{~m}, 2 \mathrm{H}), 3.22-3.37(\mathrm{~m}, 1 \mathrm{H}), 4.49-4.61(\mathrm{~m}, 1 \mathrm{H}), 4.83-4.90$ $(\mathrm{m}, 1 \mathrm{H}), 5.57-5.74(\mathrm{~m}, 1 \mathrm{H}), 7.00-7.23(\mathrm{~m}, 5 \mathrm{H}), 7.24-7.48(\mathrm{~m}, 6 \mathrm{H}), 7.60-7.71(\mathrm{~m}, 2 \mathrm{H}), 7.75-7.85$ $(\mathrm{m}, 2 \mathrm{H}) .{ }^{13} \mathrm{C} \mathrm{NMR}\left(\mathrm{CDCl}_{3}, 75 \mathrm{MHz}\right): \delta 35.14\left(\mathrm{~d}, J_{\mathrm{P}-\mathrm{C}} 3.6 \mathrm{~Hz}\right), 43.26\left(\mathrm{~d}, J_{\mathrm{P}-\mathrm{C}} 32.5 \mathrm{~Hz}\right), 120.48(\mathrm{~d}$, $\left.J_{\mathrm{P}-\mathrm{C}} 10.3 \mathrm{~Hz}\right), 126.29,128.03\left(\mathrm{~d}, J_{\mathrm{P}-\mathrm{C}} 54.3 \mathrm{~Hz}\right), 128.06\left(\mathrm{~d}, J_{\mathrm{P}-\mathrm{C}} 56.0 \mathrm{~Hz}\right), 128.20,128.41\left(\mathrm{~d}, J_{\mathrm{P}-\mathrm{C}}\right.$ $9.8 \mathrm{~Hz}), 128.89$ (d, $\left.J_{\mathrm{P}-\mathrm{C}} 10.1 \mathrm{~Hz}\right), 128.92,131.13\left(\mathrm{~d}, J_{\mathrm{P}-\mathrm{C}} 2.3 \mathrm{~Hz}\right), 131.38$ (d, $\left.J_{\mathrm{P}-\mathrm{C}} 2.6 \mathrm{~Hz}\right), 132.69$ $\left(\mathrm{d}, J_{\mathrm{P}-\mathrm{C}} 8.9 \mathrm{~Hz}\right), 132.76\left(\mathrm{~d}, J_{\mathrm{P}-\mathrm{C}} 2.6 \mathrm{~Hz}\right), 132.92\left(\mathrm{~d}, J_{\mathrm{P}-\mathrm{C}} 8.6 \mathrm{~Hz}\right), 139.18\left(\mathrm{~d}, J_{\mathrm{P}-\mathrm{C}} 13.8 \mathrm{~Hz}\right) .{ }^{31} \mathrm{P}$ $\mathrm{NMR}\left(\mathrm{CDCl}_{3}, 121.5 \mathrm{MHz}\right): \delta 21.96(\mathrm{~m})$. Elem. anal. for $\mathrm{C}_{22} \mathrm{H}_{24} \mathrm{BP}$ : calc. C 80.02, H 7.33; found C 79.91, H 7.14.

Diphenyl-(4-phenylbut-1-en-1-yl)phosphine-borane (3bb). Yield $13 \%$ from $0.081 \mathrm{~g}(0.33$ mmol) of 1b, purified with hexane/EtOAc (50/1), colorless oil. ${ }^{1} \mathrm{H}$ NMR $\left(\mathrm{CDCl}_{3}, 300 \mathrm{MHz}\right): \delta$ 0.33-1.63 (bm, $\left.\mathrm{BH}_{3}, 3 \mathrm{H}\right), 2.23-2.34(\mathrm{~m}, 2 \mathrm{H}), 2.60-2.71(\mathrm{~m}, 2 \mathrm{H}), 6.02-6.15(\mathrm{~m}, 1 \mathrm{H}), 6.57-6.76$ $(\mathrm{m}, 1 \mathrm{H}), 7.05-7.27(\mathrm{~m}, 5 \mathrm{H}), 7.34-7.48(\mathrm{~m}, 6 \mathrm{H}), 7.61-7.77(\mathrm{~m}, 4 \mathrm{H}) .{ }^{13} \mathrm{C} \mathrm{NMR}\left(\mathrm{CDCl}_{3}, 75 \mathrm{MHz}\right)$ : $\delta 34.20\left(\mathrm{~d}, J_{\mathrm{P}-\mathrm{C}} 1.2 \mathrm{~Hz}\right), 36.44\left(\mathrm{~d}, J_{\mathrm{P}-\mathrm{C}} 15.2 \mathrm{~Hz}\right), 119.40\left(\mathrm{~d}, J_{\mathrm{P}-\mathrm{C}} 57.8 \mathrm{~Hz}\right), 126.05,128.16,128.62$ $\left(\mathrm{d}, J_{\mathrm{P}-\mathrm{C}} 10.1 \mathrm{~Hz}\right), 128.49,130.90\left(\mathrm{~d}, J_{\mathrm{P}-\mathrm{C}} 2.3 \mathrm{~Hz}\right), 132.32$ (d, $\left.J_{\mathrm{P}-\mathrm{C}} 9.5 \mathrm{~Hz}\right), 151.77$ (d, $\left.J_{\mathrm{P}-\mathrm{C}} 7.5 \mathrm{~Hz}\right)$. ${ }^{31} \mathrm{P}$ NMR $\left(\mathrm{CDCl}_{3}, 121.5 \mathrm{MHz}\right): \delta 13.35(\mathrm{~m})$. Elem. anal. for $\mathrm{C}_{22} \mathrm{H}_{24} \mathrm{BP}$ : calc. C 80.02, $\mathrm{H} 7.33$; found C 80.24, H 7.50 .

2,5-Dibenzyl-3-phospholene-borane (4cb). Yield 73\% from $0.051 \mathrm{~g}(0.29 \mathrm{mmol})$ of 1c, purified with hexane/EtOAc (50:1), white solid, m.p. 100-102 ${ }^{\circ} \mathrm{C} .{ }^{1} \mathrm{H} \mathrm{NMR}\left(\mathrm{CDCl}_{3}, 300 \mathrm{MHz}\right)$ : $\delta$ 0.12-1.63 (bm, $\left.\mathrm{BH}_{3}, 3 \mathrm{H}\right), 2.57-2.72(\mathrm{~m}, 2 \mathrm{H}), 3.08-3.31(\mathrm{~m}, 4 \mathrm{H}), 5.65-5.79(\mathrm{~m}, 2 \mathrm{H}), 7.04-7.28$ $(\mathrm{m}, 15 \mathrm{H}) .{ }^{13} \mathrm{C} \mathrm{NMR}\left(\mathrm{CDCl}_{3}, 75 \mathrm{MHz}\right): \delta 38.08\left(\mathrm{~d}, J_{\mathrm{P}-\mathrm{C}} 5.2 \mathrm{~Hz}\right), 46.62\left(\mathrm{~d}, J_{\mathrm{P}-\mathrm{C}} 32.2 \mathrm{~Hz}\right) 126.38$, 128.24, 128.44, 128.83, 128.84 (d, $\left.J_{\mathrm{P}-\mathrm{C}} 9.5 \mathrm{~Hz}\right), 131.20$ (d, $\left.J_{\mathrm{P}-\mathrm{C}} 8.6 \mathrm{~Hz}\right), 131.26$ (d, $\left.J_{\mathrm{P}-\mathrm{C}} 48.6 \mathrm{~Hz}\right)$, $131.49\left(\mathrm{~d}, J_{\mathrm{P}-\mathrm{C}} 2.3 \mathrm{~Hz}\right), 132.60\left(\mathrm{~d}, J_{\mathrm{P}-\mathrm{C}} 4.3 \mathrm{~Hz}\right), 139.97\left(\mathrm{~d}, J_{\mathrm{P}-\mathrm{C}} 13.5 \mathrm{~Hz}\right) .{ }^{31} \mathrm{P} \mathrm{NMR}\left(\mathrm{CDCl}_{3}, 121.5\right.$ $\mathrm{MHz}$ ): $\delta 38.66(\mathrm{~m})$. Elem. anal. for $\mathrm{C}_{24} \mathrm{H}_{26} \mathrm{BP}$ : calc. C 80.91, H 7.36; found C 80.92, H 7.50.

$\boldsymbol{t}$-Butyl-(hexa-1,5-dien-3-yl)phenylphosphine-borane (2ac). Yield 13\% from $0.148 \mathrm{~g}(0.76$ mmol) of 1a, purified with hexane/EtOAc (10/1), colorless oil. ${ }^{1} \mathrm{H}$ NMR $\left(\mathrm{CDCl}_{3}, 300 \mathrm{MHz}\right)$ : $\delta$ 0.07-1.20 (bm, BH $3,3 \mathrm{H}), 1.04$ (d, $\left.J_{\mathrm{P}-\mathrm{H}} 13.7 \mathrm{~Hz}, t-\mathrm{Bu}, 9 \mathrm{H}\right), 1.81-1.95(\mathrm{~m}, 1 \mathrm{H}), 2.07-2.22(\mathrm{~m}, 1 \mathrm{H})$, 3.00-3.14 (m, 1H), 4.72-4.91 (m, 2H), 5.12-5.25 (m, 2H), 5.48-5.64 (m, 1H), 5.86-6.02 (m, 1H), 7.31-7.47 (m, 3H), 7.62-7.74 (m, 2H). ${ }^{31} \mathrm{P} \mathrm{NMR}\left(\mathrm{CDCl}_{3}, 121.5 \mathrm{MHz}\right): \delta 36.69(\mathrm{~m})$. Elem. anal. for $\mathrm{C}_{16} \mathrm{H}_{26} \mathrm{BP}$ : calc. C 73.87, $\mathrm{H} 10.07$; found $\mathrm{C} 74.15, \mathrm{H} 10.34$.

$\boldsymbol{t}$-Butyl-(hexa-1,5-dien-1-yl)phenylphosphine-borane (3ac). Yield 62\% from $0.148 \mathrm{~g}(0.76$ mmol) of 1a, purified with hexane/EtOAc (10/1), colorless oil. ${ }^{1} \mathrm{H}$ NMR $\left(\mathrm{CDCl}_{3}, 300 \mathrm{MHz}\right)$ : $\delta$ 0.08-1.23 (bm, BH $3,3 \mathrm{H}), 1.02$ (d, $\left.J_{\mathrm{P}-\mathrm{H}} 14.0 \mathrm{~Hz}, t-\mathrm{Bu}, 9 \mathrm{H}\right), 2.13-2.23(\mathrm{~m}, 2 \mathrm{H}), 2.29-2.39$ (m, 2H), 4.90-5.03 (m, 2H), 5.64-5.81 (m, 1H), 6.10-6.22 (m, 1H), 6.69-6.87 (m, 1H), 7.29-7.45 (m, 3H), 7.60-7.72 (m, 2H). ${ }^{13} \mathrm{C} \mathrm{NMR}\left(\mathrm{CDCl}_{3}, 75 \mathrm{MHz}\right): \delta 25.36\left(\mathrm{~d}, J_{\mathrm{P}-\mathrm{C}} 2.9 \mathrm{~Hz}\right), 29.36\left(\mathrm{~d}, J_{\mathrm{P}-\mathrm{C}} 35.1 \mathrm{~Hz}\right)$, $32.10\left(\mathrm{~d}, J_{\mathrm{P}-\mathrm{C}} 1.2 \mathrm{~Hz}\right), 34.39$ (d, $\left.J_{\mathrm{P}-\mathrm{C}} 14.9 \mathrm{~Hz}\right), 114.28\left(\mathrm{~d}, J_{\mathrm{P}-\mathrm{C}} 55.8 \mathrm{~Hz}\right), 115.54,128.11\left(\mathrm{~d}, J_{\mathrm{P}-\mathrm{C}} 9.5\right.$ $\mathrm{Hz}), 128.30$ (d, J $\left.J_{\mathrm{P}-\mathrm{C}} 52.6 \mathrm{~Hz}\right), 130.77$ (d, $J_{\mathrm{P}-\mathrm{C}} 2.3 \mathrm{~Hz}$ ), 133.06 (d, $J_{\mathrm{P}-\mathrm{C}} 8.3 \mathrm{~Hz}$ ), 137.13, 153.97 (d, $\left.J_{\mathrm{P}-\mathrm{C}} 8.9 \mathrm{~Hz}\right) .{ }^{31} \mathrm{P} \mathrm{NMR}\left(\mathrm{CDCl}_{3}, 121.5 \mathrm{MHz}\right): \delta 25.19(\mathrm{~m})$. Elem. anal. for $\mathrm{C}_{16} \mathrm{H}_{26} \mathrm{BP}$ : calc. C 73.87, H 10.07; found C 73.99, H 10.00. 
(Hexa-1,5-dien-3-yl)diphenylphosphine-borane (2bc). Yield 42\%, from $0.072 \mathrm{~g}$ (0.30 mmol) of 1b, purified with hexane/EtOAc (6/1), colorless oil. ${ }^{1} \mathrm{H}$ NMR $\left(\mathrm{CDCl}_{3}, 300 \mathrm{MHz}\right): \delta 0.30-1.58$ (bm, $\left.\mathrm{BH}_{3}, 3 \mathrm{H}\right), 2.11-2.20(\mathrm{~m}, 2 \mathrm{H}), 3.02-3.18(\mathrm{~m}, 1 \mathrm{H}), 4.72-5.04(\mathrm{~m}, 2 \mathrm{H}), 5.52-5.79(\mathrm{~m}, 1 \mathrm{H})$, 5.98-6.13 (m, 1H), 6.44-6.64 (m, 1H), 7.26-7.45 (m, 6H), 7.58-7.75 (m, 4H). ${ }^{13} \mathrm{C} \mathrm{NMR}\left(\mathrm{CDCl}_{3}\right.$, $75 \mathrm{MHz}): \delta 33.04\left(\mathrm{~d}, J_{\mathrm{P}-\mathrm{C}} 3.2 \mathrm{~Hz}\right), 41.01\left(\mathrm{~d}, J_{\mathrm{P}-\mathrm{C}} 33.9 \mathrm{~Hz}\right), 115.60,119.94\left(\mathrm{~d}, J_{\mathrm{P}-\mathrm{C}} 10.6 \mathrm{~Hz}\right)$, $128.39\left(\mathrm{~d}, J_{\mathrm{P}-\mathrm{C}} 9.8 \mathrm{~Hz}\right), 128.80\left(\mathrm{~d}, J_{\mathrm{P}-\mathrm{C}} 10.1 \mathrm{~Hz}\right), 130.00\left(\mathrm{~d}, J_{\mathrm{P}-\mathrm{C}} 59.2 \mathrm{~Hz}\right), 131.11\left(\mathrm{~d}, J_{\mathrm{P}-\mathrm{C}} 2.3 \mathrm{~Hz}\right)$, $131.27\left(\mathrm{~d}, J_{\mathrm{P}-\mathrm{C}} 2.3 \mathrm{~Hz}\right), 132.60\left(\mathrm{~d}, J_{\mathrm{P}-\mathrm{C}} 8.6 \mathrm{~Hz}\right), 132.94$ (d, $\left.J_{\mathrm{P}-\mathrm{C}} 8.6 \mathrm{~Hz}\right), 133.09$ (d, $\left.J_{\mathrm{P}-\mathrm{C}} 2.9 \mathrm{~Hz}\right)$, $135.50\left(\mathrm{~d}, J_{\mathrm{P}-\mathrm{C}} 14.1 \mathrm{~Hz}\right) .{ }^{31} \mathrm{P} \mathrm{NMR}\left(\mathrm{CDCl}_{3}, 121.5 \mathrm{MHz}\right): \delta 21.99(\mathrm{~m})$. Elem. anal. for $\mathrm{C}_{18} \mathrm{H}_{22} \mathrm{BP}$ (as a mixture with 3bc): calc. C 77.17, H 7.92; found C 77.30, H 7.80.

(Hexa-1,5-dien-1-yl)diphenylphosphine-borane (3bc). Yield 42\%, from $0.072 \mathrm{~g}(0.30 \mathrm{mmol})$ of $\mathbf{1 b}$, purified with hexane/EtOAc (6/1), colorless oil. ${ }^{1} \mathrm{H} \mathrm{NMR}\left(\mathrm{CDCl}_{3}, 300 \mathrm{MHz}\right): \delta 0.30-1.58$ (bm, $\left.\mathrm{BH}_{3}, 3 \mathrm{H}\right), 2.01-2.38(\mathrm{~m}, 4 \mathrm{H}), 4.72-5.04(\mathrm{~m}, 4 \mathrm{H}), 5.52-5.79(\mathrm{~m}, 2 \mathrm{H}), 7.26-7.45(\mathrm{~m}, 6 \mathrm{H})$, 7.47-7.58 (m, 4H). ${ }^{13} \mathrm{C} \mathrm{NMR}\left(\mathrm{CDCl}_{3}, 75 \mathrm{MHz}\right): \delta 32.04$ (d, $\left.J_{\mathrm{P}-\mathrm{C}} 1.2 \mathrm{~Hz}\right), 34.00\left(\mathrm{~d}, J_{\mathrm{P}-\mathrm{C}} 15.2 \mathrm{~Hz}\right)$, 116.74, 118.87 (d, $\left.J_{\mathrm{P}-\mathrm{C}} 58.3 \mathrm{~Hz}\right), 128.07$ (d, $\left.J_{\mathrm{P}-\mathrm{C}} 63.2 \mathrm{~Hz}\right), 128.67$ (d, $\left.J_{\mathrm{P}-\mathrm{C}} 10.1 \mathrm{~Hz}\right), 130.96\left(\mathrm{~d}, J_{\mathrm{P}-}\right.$ C $2.6 \mathrm{~Hz}), 132.35$ (d, $\left.J_{\mathrm{P}-\mathrm{C}} 9.8 \mathrm{~Hz}\right), 137.01,152.21$ (d, $\left.J_{\mathrm{P}-\mathrm{C}} 7.8 \mathrm{~Hz}\right) .{ }^{31} \mathrm{P} \mathrm{NMR}\left(\mathrm{CDCl}_{3}, 121.5 \mathrm{MHz}\right)$ : $\delta 13.46(\mathrm{~m})$. Elem. anal. for $\mathrm{C}_{18} \mathrm{H}_{22} \mathrm{BP}$ (as a mixture with 2bc): calc. C 77.17, $\mathrm{H}$ 7.92; found $\mathrm{C}$ 77.30, H 7.80 .

2-Allyl-1-phenyl-3-phospholene-borane (2cc). Yield 51\% from $0.051 \mathrm{~g}(0.29 \mathrm{mmol})$ of 1c, purified with hexane/EtOAc (50:1), colorless oil. ${ }^{1} \mathrm{H} \mathrm{NMR}\left(\mathrm{CDCl}_{3}, 300 \mathrm{MHz}\right): \delta$ 0.09-1.31 (bm, $\left.\mathrm{BH}_{3}, 3 \mathrm{H}\right), 2.10-2.29$ (m, 2H), 2.50-2.69 (m, 2H), 2.84-2.92 (m, ,-CH-P-CH, $\left.1 \mathrm{H}\right)$, 2.83-3.01 (m, $1 \mathrm{H}), 4.91-5.06(\mathrm{~m} \mathrm{2H}), 5.68-5.86(\mathrm{~m}, 1 \mathrm{H}), 5.86-6.00(\mathrm{~m}, 2 \mathrm{H}), 7.29-7.48(\mathrm{~m}, 3 \mathrm{H}), 7.58-7.72(\mathrm{~m}$, 2H). ${ }^{13} \mathrm{C} \mathrm{NMR}\left(\mathrm{CDCl}_{3}, 75 \mathrm{MHz}\right): \delta 33.04\left(\mathrm{~d}, J_{\mathrm{P}-\mathrm{C}} 35.9 \mathrm{~Hz}\right), 35.39\left(\mathrm{~d}, J_{\mathrm{P}-\mathrm{C}} 6.0 \mathrm{~Hz}\right), 44.79\left(\mathrm{~d}, J_{\mathrm{P}-\mathrm{C}}\right.$ $33.6 \mathrm{~Hz}), 116.85,127.56$ (d, $\left.J_{\mathrm{P}-\mathrm{C}} 0.6 \mathrm{~Hz}\right), 128.83$ (d, $\left.J_{\mathrm{P}-\mathrm{C}} 9.8 \mathrm{~Hz}\right), 131.15$ (d, J $\left.J_{\mathrm{P}-\mathrm{C}} 8.9 \mathrm{~Hz}\right), 131.24$ (d, $\left.J_{\mathrm{P}-\mathrm{C}} 48.3 \mathrm{~Hz}\right), 131.90$ (d, $\left.J_{\mathrm{P}-\mathrm{C}} 2.6 \mathrm{~Hz}, \mathrm{P}-\mathrm{Ph}\right), 133.76$ (d, $\left.J_{\mathrm{P}-\mathrm{C}} 5.2 \mathrm{~Hz}\right), 135.89$ (d, J $\left.J_{\mathrm{P}-\mathrm{C}} 11.5 \mathrm{~Hz}\right)$. ${ }^{31} \mathrm{P}$ NMR $\left(\mathrm{CDCl}_{3}, 121.5 \mathrm{MHz}\right): \delta 31.44(\mathrm{~m})$. Elem. anal. for $\mathrm{C}_{13} \mathrm{H}_{18} \mathrm{BP}$ : calc. C 72.26, H 8.40; found $\mathrm{C} 72.40, \mathrm{H} 8.45$.

2,5-Diallyl-1-phenyl-3-phospholene-borane (4cc). Yield $25 \%$ from $0.051 \mathrm{~g}(0.29 \mathrm{mmol})$ of 1c, purified with hexane/EtOAc (50:1), colorless oil. ${ }^{1} \mathrm{H} \mathrm{NMR}\left(\mathrm{CDCl}_{3}, 300 \mathrm{MHz}\right)$ : $\delta$ 0.06-1.27 (bm, $\left.\mathrm{BH}_{3}, 3 \mathrm{H}\right), 2.10-2.27(\mathrm{~m}, 2 \mathrm{H}), 2.50-2.66(\mathrm{~m}, 2 \mathrm{H}), 2.88-3.00(\mathrm{~m}, 2 \mathrm{H}), 4.92-5.05(\mathrm{~m}, 4 \mathrm{H}), 5.67-$ $5.84(\mathrm{~m}, 2 \mathrm{H}), 5.88-6.01(\mathrm{~m}, 2 \mathrm{H}), 7.31-7.47(\mathrm{~m}, 3 \mathrm{H}), 7.61-7.73(\mathrm{~m}, 2 \mathrm{H}) .{ }^{13} \mathrm{C} \mathrm{NMR}\left(\mathrm{CDCl}_{3}, 75\right.$ MHz): $\delta 35.85$ (d, $\left.J_{\mathrm{P}-\mathrm{C}} 4.3 \mathrm{~Hz}\right), 44.75$ (d, $\left.J_{\mathrm{P}-\mathrm{C}} 33.1 \mathrm{~Hz}\right), 116.86,128.83$ (d, J $\left.J_{\mathrm{P}-\mathrm{C}} 9.5 \mathrm{~Hz}\right), 131.23$ (d, $\left.J_{\mathrm{P}-\mathrm{C}} 8.6 \mathrm{~Hz}\right), 131.41\left(\mathrm{~d}, J_{\mathrm{P}-\mathrm{C}} 2.6 \mathrm{~Hz}\right), 131.51\left(\mathrm{~d}, J_{\mathrm{P}-\mathrm{C}} 48.9 \mathrm{~Hz}\right), 132.62\left(\mathrm{~d}, J_{\mathrm{P}-\mathrm{C}} 4.3 \mathrm{~Hz}\right), 135.85(\mathrm{~d}$, $\left.J_{\mathrm{P}-\mathrm{C}} 11.5 \mathrm{~Hz}\right) .{ }^{31} \mathrm{P} \mathrm{NMR}\left(\mathrm{CDCl}_{3}, 121.5 \mathrm{MHz}\right): \delta 38.54(\mathrm{~m})$. Elem. anal. for $\mathrm{C}_{16} \mathrm{H}_{22} \mathrm{BP}$ : calc. C 75.03, H 8.66; found C 74.95, H 8.60.

t-Butyl-(3-cyclohexylprop-1-en-1-yl)phenylphosphine-borane (3ad). Yield 47\% from $0.043 \mathrm{~g}$ $(0.2 \mathrm{mmol})$ of 1a, purified with hexane/EtOAc (10/1), colorless oil. ${ }^{1} \mathrm{H} \mathrm{NMR}\left(\mathrm{CDCl}_{3}, 300 \mathrm{MHz}\right)$ : $\delta$ 0.08-1.24 (bm, BH $3,3 \mathrm{H}), 1.02$ (d $\left.J_{\mathrm{P}-\mathrm{H}} 14.1 \mathrm{~Hz}, t-\mathrm{Bu}, 9 \mathrm{H}\right), 1.09-1.20$ (bm, 4H), 1.30-1.44 (bm, $1 \mathrm{H}), 1.50-1.70(\mathrm{bm}, 6 \mathrm{H}), 2.10-2.17(\mathrm{~m}, 2 \mathrm{H}), 6.06-6.17(\mathrm{~m}, 1 \mathrm{H}), 6.67-6.86(\mathrm{~m}, 1 \mathrm{H}), 7.30-7.45(\mathrm{~m}$, $3 \mathrm{H}), 7.60-7.71(\mathrm{~m}, 2 \mathrm{H}) .{ }^{13} \mathrm{C} \mathrm{NMR}\left(\mathrm{CDCl}_{3}, 75 \mathrm{MHz}\right): \delta 25.36\left(\mathrm{~d}, J_{\mathrm{P}-\mathrm{C}} 2.9 \mathrm{~Hz}\right), 26.12,26.30$, $29.33\left(\mathrm{~d}, J_{\mathrm{P}-\mathrm{C}} 35.1 \mathrm{~Hz}\right), 30.85,33.02,33.11,37.22\left(\mathrm{~d}, J_{\mathrm{P}-\mathrm{C}} 1.2 \mathrm{~Hz}\right), 43.43$ (d, J $\left.J_{\mathrm{P}-\mathrm{C}} 14.4 \mathrm{~Hz}\right), 114.50$ 
$\left(\mathrm{d}, J_{\mathrm{P}-\mathrm{C}} 54.9 \mathrm{~Hz}\right), 128.07\left(\mathrm{~d}, J_{\mathrm{P}-\mathrm{C}} 9.5 \mathrm{~Hz}\right), 128.44\left(\mathrm{~d}, J_{\mathrm{P}-\mathrm{C}} 55.2 \mathrm{~Hz}\right), 130.70\left(\mathrm{~d}, J_{\mathrm{P}-\mathrm{C}} 2.6 \mathrm{~Hz}\right), 133.00$ $\left(\mathrm{d}, J_{\mathrm{P}-\mathrm{C}} 8.1 \mathrm{~Hz}\right), 154.08\left(\mathrm{~d}, J_{\mathrm{P}-\mathrm{C}} 9.5 \mathrm{~Hz}\right) .{ }^{31} \mathrm{P} \mathrm{NMR}\left(\mathrm{CDCl}_{3}, 121.5 \mathrm{MHz}\right): \delta 24.85(\mathrm{~m})$. Elem. anal. for $\mathrm{C}_{18} \mathrm{H}_{30} \mathrm{BP}$ : calc. C 75.01, H 10.49; found C 74.75, H 10.22.

(3-Cyclohexylpropen-3-yl)diphenylphosphine-borane (2bd). Yield 22\% from $0.076 \mathrm{~g}(0.32$ mmol) of 1b, purified with hexane/EtOAc (6/1), colorless oil. ${ }^{1} \mathrm{H}$ NMR $\left(\mathrm{CDCl}_{3}, 300 \mathrm{MHz}\right): \delta$ 0.36-1.65 (bm, $\left.\mathrm{BH}_{3}, 3 \mathrm{H}\right), 1.31-1.49(\mathrm{~m}, 3 \mathrm{H}), 1.50-1.71(\mathrm{~m}, 8 \mathrm{H}), 2.89-3.04(\mathrm{~m}, 1 \mathrm{H}), 4.71-5.00$ $(\mathrm{m}, 2 \mathrm{H}), 5.64-5.83(\mathrm{~m}, 1 \mathrm{H}), 7.24-7.47(\mathrm{~m}, 6 \mathrm{H}), 7.5-7.80(\mathrm{~m}, 4 \mathrm{H}) .{ }^{13} \mathrm{C} \mathrm{NMR}\left(\mathrm{CDCl}_{3}, 75 \mathrm{MHz}\right): \delta$ 25.92, 26.45, 30.64 (d, $J_{\text {P-C }} 2.9$ Hz), 33.59 (d, $J_{\text {P-C }} 9.5$ Hz), 37.19, 47.43 (d, $J_{\text {P-C }} 31.9$ Hz), 120.03 $\left(\mathrm{d}, J_{\mathrm{P}-\mathrm{C}} 11.8 \mathrm{~Hz}\right), 128.19\left(\mathrm{~d}, J_{\mathrm{P}-\mathrm{C}} 9.8 \mathrm{~Hz}\right), 128.66\left(\mathrm{~d}, J_{\mathrm{P}-\mathrm{C}} 9.8 \mathrm{~Hz}\right), 129.35$ (d, $\left.J_{\mathrm{P}-\mathrm{C}} 58.3 \mathrm{~Hz}\right), 129.39$ $\left(\mathrm{d}, J_{\mathrm{P}-\mathrm{C}} 52.9 \mathrm{~Hz}\right), 130.24\left(\mathrm{~d}, J_{\mathrm{P}-\mathrm{C}} 2.6 \mathrm{~Hz}\right), 130.98\left(\mathrm{~d}, J_{\mathrm{P}-\mathrm{C}} 2.6 \mathrm{~Hz}\right), 132.15\left(\mathrm{~d}, J_{\mathrm{P}-\mathrm{C}} 2.9 \mathrm{~Hz}\right), 132.40$ $\left(\mathrm{d}, J_{\mathrm{P}-\mathrm{C}} 7.8 \mathrm{~Hz}\right), 133.07\left(\mathrm{~d}, J_{\mathrm{P}-\mathrm{C}} 8.1 \mathrm{~Hz}\right) .{ }^{31} \mathrm{P} \mathrm{NMR}\left(\mathrm{CDCl}_{3}, 121.5 \mathrm{MHz}\right): \delta 19.36(\mathrm{~m})$. Elem. anal. for $\mathrm{C}_{21} \mathrm{H}_{28} \mathrm{BP}$ (as a mixture with 9d): calc. C 78.27, $\mathrm{H} 8.76$; found $\mathrm{C} 78.45, \mathrm{H} 8.70$.

(3-Cyclohexylpropen-1-yl)diphenylphosphine-borane (3bd). Yield 47\% from $0.076 \mathrm{~g}(0.32$ mmol) of 1b, purified with hexane/EtOAc (6/1), colorless oil. ${ }^{1} \mathrm{H}$ NMR $\left(\mathrm{CDCl}_{3}, 300 \mathrm{MHz}\right): \delta$ 0.36-1.65 (bm, $\left.\mathrm{BH}_{3}, 3 \mathrm{H}\right), 1.31-1.49$ (m, 3H), 1.50-1.71 (m, 8H), 2.08-2.18 (m, 2H), 5.97-6.13 $(\mathrm{m}, 1 \mathrm{H}), 6.47-6.68(\mathrm{~m}, 1 \mathrm{H}), 7.24-7.47(\mathrm{~m}, 6 \mathrm{H}), 7.49-7.62(\mathrm{~m}, 4 \mathrm{H}) .{ }^{13} \mathrm{C} \mathrm{NMR}\left(\mathrm{CDCl}_{3}, 75 \mathrm{MHz}\right)$ : $\delta$ 26.12, 26.29, 33.07, 38.24 (d, $\left.J_{\mathrm{P}-\mathrm{C}} 2.9 \mathrm{~Hz}\right), 43.00$ (d, $\left.J_{\mathrm{P}-\mathrm{C}} 15.2 \mathrm{~Hz}\right), 119.05$ (d, $\left.J_{\mathrm{P}-\mathrm{C}} 58.3 \mathrm{~Hz}\right)$, 128.7 (d, $\left.J_{\mathrm{P}-\mathrm{C}} 9.77 \mathrm{~Hz}\right), 130.24\left(\mathrm{~d}, J_{\mathrm{P}-\mathrm{C}} 63.2 \mathrm{~Hz}\right), 130.90$ (d, $\left.J_{\mathrm{P}-\mathrm{C}} 2.3 \mathrm{~Hz}\right), 132.29$ (d, $\left.J_{\mathrm{P}-\mathrm{C}} 9.2 \mathrm{~Hz}\right)$, $152.33\left(\mathrm{~d}, J_{\mathrm{P}-\mathrm{C}} 7.5 \mathrm{~Hz}\right) .{ }^{31} \mathrm{P} \mathrm{NMR}\left(\mathrm{CDCl}_{3}, 121.5 \mathrm{MHz}\right): \delta 13.13(\mathrm{~m})$. Elem. anal. for $\mathrm{C}_{21} \mathrm{H}_{28} \mathrm{BP}$ (as a mixture with 8d): calc. C 78.27, H 8.76; found C 78.45, H 8.70.

t-Butylphenyl-(3-trimethylsilylprop-1-en-1-yl)phosphine-borane (3ae). Yield 91\% from $0.049 \mathrm{~g}(0.22 \mathrm{mmol})$ of $\mathbf{1 a}$, purified with hexane/EtOAc (6/1), white solid. ${ }^{1} \mathrm{H} \mathrm{NMR}\left(\mathrm{CDCl}_{3}, 300\right.$ $\mathrm{MHz}): \delta 0.05$ (s, 9H, TMS), 0.57-1.30 (bm, BH $3,3 \mathrm{H}), 1.09$ (d, $\left.J_{\mathrm{P}-\mathrm{H}} 13.7 \mathrm{~Hz}, t-\mathrm{Bu}, 9 \mathrm{H}\right), 1.84-1.89$ $(\mathrm{m}, 2 \mathrm{H}), 5.95-6.05(\mathrm{~m}, 1 \mathrm{H}), 6.80-6.99(\mathrm{~m}, 1 \mathrm{H}), 7.36-7.47(\mathrm{~m}, 3 \mathrm{H}), 7.68-7.77(\mathrm{~m}, 2 \mathrm{H}) .{ }^{13} \mathrm{C} \mathrm{NMR}$ $\left(\mathrm{CDCl}_{3}, 75 \mathrm{MHz}\right): \delta-1.83,25.35\left(\mathrm{~d}, J_{\mathrm{P}-\mathrm{C}} 2.9 \mathrm{~Hz}\right), 28.00\left(\mathrm{~d}, J_{\mathrm{P}-\mathrm{C}} 14.1 \mathrm{~Hz}\right), 29.32\left(\mathrm{~d}, J_{\mathrm{P}-\mathrm{C}} 36.2 \mathrm{~Hz}\right)$, $110.24\left(\mathrm{~d}, J_{\mathrm{P}-\mathrm{C}} 58.3 \mathrm{~Hz}\right), 127.98\left(\mathrm{~d}, J_{\mathrm{P}-\mathrm{C}} 8.9 \mathrm{~Hz}\right), 128.94\left(\mathrm{~d}, J_{\mathrm{P}-\mathrm{C}} 52.3 \mathrm{~Hz}\right), 130.55\left(\mathrm{~d}, J_{\mathrm{P}-\mathrm{C}} 2.9\right.$ $\mathrm{Hz}), 132.95$ (d, $\left.J_{\mathrm{P}-\mathrm{C}} 8.3 \mathrm{~Hz}\right), 153.11\left(\mathrm{~d}, J_{\mathrm{P}-\mathrm{C}} 10.1 \mathrm{~Hz}\right) .{ }^{31} \mathrm{P} \mathrm{NMR}\left(\mathrm{CDCl}_{3}, 121.5 \mathrm{MHz}\right): \delta 24.90$ (m). Elem. anal. for $\mathrm{C}_{16} \mathrm{H}_{30} \mathrm{BPSi}$ : calc. C 65.75, H 10.35; found C 66.02, H 10.20.

3-(t-Butylphenylboranatophosphinyl)-pent-4-enoic acid ethyl ester (2af). Yield 23\% from $0.101 \mathrm{~g}(0.46 \mathrm{mmol})$ of 1a, 3:1 inseparable mixture of diastereoisomers, purified with hexane/EtOAc (6/1), colorless oil.

Major: ${ }^{1} \mathrm{H}$ NMR $\left(\mathrm{CDCl}_{3}, 300 \mathrm{MHz}\right): \delta$ 0.15-1.56 (bm, $\left.\mathrm{BH}_{3}, 3 \mathrm{H}\right), 1.15\left(\mathrm{~d}, J_{\mathrm{P}-\mathrm{H}} 13.9 \mathrm{~Hz}, t\right.$-Bu, 9H), $1.28\left(\mathrm{t}, J_{\mathrm{H}-\mathrm{H}} 7.0 \mathrm{~Hz}, 3 \mathrm{H}\right), 2.34\left(\mathrm{ddd}, J_{\mathrm{H}-\mathrm{H}} 9.8 \mathrm{~Hz}, J_{\mathrm{H}-\mathrm{H}} 12.0 \mathrm{~Hz}, J_{\mathrm{P}-\mathrm{H}} 2.5 \mathrm{~Hz}, 2 \mathrm{H}\right), 3.71-3.87$ $(\mathrm{m}, 1 \mathrm{H}), 3.99-4.12(\mathrm{~m}, 3 \mathrm{H}), 5.23-5.30(\mathrm{~m}, 1 \mathrm{H}), 5.35-5.44(\mathrm{~m}, 1 \mathrm{H}), 5.99-6.15(\mathrm{~m}, 1 \mathrm{H}), 7.43-7.53$ (m, 3H), 7.75-7.83 (m, 2H). ${ }^{31} \mathrm{P} \mathrm{NMR}\left(\mathrm{CDCl}_{3}, 121.5 \mathrm{MHz}\right): 37.20(\mathrm{~m})$.

Minor: ${ }^{1} \mathrm{H}$ NMR $\left(\mathrm{CDCl}_{3}, 300 \mathrm{MHz}\right): \delta$ 0.15-1.56 (bm, $\left.\mathrm{BH}_{3}, 3 \mathrm{H}\right), 1.04\left(\mathrm{~d}, J_{\mathrm{P}-\mathrm{H}} 14.0 \mathrm{~Hz}, t\right.$-Bu, $9 \mathrm{H}), 1.10\left(\mathrm{t}, J_{\mathrm{H}-\mathrm{H}} 6.9 \mathrm{~Hz}, 3 \mathrm{H}\right), 2.15-2.28(\mathrm{~m}, 1 \mathrm{H}), 2.42-2.57(\mathrm{~m}, 1 \mathrm{H}), 3.14-3.29(\mathrm{~m}, 1 \mathrm{H}), 4.15-$ $4.33(\mathrm{~m}, 2 \mathrm{H}), 5.05-5.22(\mathrm{~m}, 2 \mathrm{H}), 5.88-6.06(\mathrm{~m}, 1 \mathrm{H}), 7.39-7.50(\mathrm{~m}, 3 \mathrm{H}), 7.64-7.74(\mathrm{~m}, 2 \mathrm{H}) .{ }^{31} \mathrm{P}$ NMR $\left(\mathrm{CDCl}_{3}, 121.5 \mathrm{MHz}\right): \delta 36.87(\mathrm{~m})$. Elem. anal. for $\mathrm{C}_{17} \mathrm{H}_{28} \mathrm{BO}_{2} \mathrm{P}$ : calc. C 66.69, H 9.22; found C 66.40, H 9.35. 
3-(t-Butylphenylboranatophosphinyl)pent-3-enoic acid ethyl ester (3af). Yield 20\% from $0.041 \mathrm{~g}(0.19 \mathrm{mmol})$ of 1a, colorless oil. ${ }^{1} \mathrm{H} \mathrm{NMR}\left(\mathrm{CDCl}_{3}, 300 \mathrm{MHz}\right): \delta 0.24-1.32\left(\mathrm{bm}, \mathrm{BH}_{3}\right.$, $3 \mathrm{H}), 1.08\left(\mathrm{~d}, J_{\mathrm{P}-\mathrm{H}} 14.0 \mathrm{~Hz}, t-\mathrm{Bu}, 9 \mathrm{H}\right), 1.23\left(\mathrm{t}, J_{\mathrm{H}-\mathrm{H}} 7.2 \mathrm{~Hz}, 3 \mathrm{H}\right), 2.46-2.54(\mathrm{~m}, 2 \mathrm{H}), 2.58-2.68(\mathrm{~m}$, $2 \mathrm{H}), 4.12\left(\mathrm{q}, J_{\mathrm{H}-\mathrm{H}} 7.2 \mathrm{~Hz}, 2 \mathrm{H}\right), 6.25-6.35(\mathrm{~m}, 1 \mathrm{H}), 6.75-6.92(\mathrm{~m}, 1 \mathrm{H}), 7.39-7.49(\mathrm{~m}, 3 \mathrm{H}), 7.67-$ $7.76(\mathrm{~m}, 2 \mathrm{H}) .{ }^{13} \mathrm{C} \mathrm{NMR}\left(\mathrm{CDCl}_{3}, 75 \mathrm{MHz}\right): \delta .14 .17,25.35\left(\mathrm{~d}, J_{\mathrm{P}-\mathrm{C}} 2.6 \mathrm{~Hz}\right), 29.68,32.64\left(\mathrm{~d}, J_{\mathrm{P}-\mathrm{C}}\right.$ $1.2 \mathrm{~Hz}), 60.62,115.39$ (d, $\left.J_{\mathrm{P}-\mathrm{C}} 54.3 \mathrm{~Hz}\right), 128.20$ (d, $\left.J_{\mathrm{P}-\mathrm{C}} 9.8 \mathrm{~Hz}\right), 130.89$ (d, $\left.J_{\mathrm{P}-\mathrm{C}} 2.0 \mathrm{~Hz}\right), 133.08$ $\left(\mathrm{d}, J_{\mathrm{P}-\mathrm{C}} 8.1 \mathrm{~Hz}\right), 152.24\left(\mathrm{~d}, J_{\mathrm{P}-\mathrm{C}} 11.5 \mathrm{~Hz}\right), 172.36 .{ }^{31} \mathrm{P} \mathrm{NMR}\left(\mathrm{CDCl}_{3}, 121.5 \mathrm{MHz}\right): \delta 25.30(\mathrm{~m})$. Elem. anal. for $\mathrm{C}_{17} \mathrm{H}_{28} \mathrm{BO}_{2} \mathrm{P}$ : calc. C 66.69, H 9.22; found C 66.74, H 9.10.

1,6-Bis(t-butylphenylboranatophosphinyl)-hexa-1,5-diene (5). Mixture of two diastereoisomers $\mathrm{dr} 50: 50$, yield $66 \%$ from $0.110 \mathrm{~g}(0.50 \mathrm{mmol})$ of 1a, purified with hexane/EtOAc (6/1), pasty white solid. ${ }^{1} \mathrm{H} \mathrm{NMR}\left(\mathrm{CDCl}_{3}, 300 \mathrm{MHz}\right): \delta 0.13-1.34\left(\mathrm{bm}, \mathrm{BH}_{3}, 3 \mathrm{H}\right)$, $1.02\left(\mathrm{~d}, J_{\mathrm{P}-\mathrm{H}} 14.1 \mathrm{~Hz}, t\right.$-Bu, $\left.9 \mathrm{H}\right), 1.08\left(\mathrm{~d}, J_{\mathrm{H}-\mathrm{H}} 14.0 \mathrm{~Hz}, 9 \mathrm{H}\right), 2.50-2.55(\mathrm{~m}, 4 \mathrm{H}), 6.24-6.34(\mathrm{~m}$, $2 \mathrm{H}), 6.75-6.93(\mathrm{~m}, 2 \mathrm{H}), 7.39-7.50(\mathrm{~m}, 6 \mathrm{H}), 7.66-7.76(\mathrm{~m}, 4 \mathrm{H}) .{ }^{31} \mathrm{P} \mathrm{NMR}\left(\mathrm{CDCl}_{3}, 121.5 \mathrm{MHz}\right): \delta$ 25.39 (m). Elem. anal. for $\mathrm{C}_{26} \mathrm{H}_{42} \mathrm{~B}_{2} \mathrm{OP}_{2}$ : calc. C 71.27, $\mathrm{H}$ 9.66; found C 71.45, H 9.32.

\section{General procedure for reaction between allylphosphine-borane 1 and carbonyl compounds}

In a Schlenk flask equipped with magnetic stirrer and argon inlet was placed allylphosphineborane $1(0.040-0.193 \mathrm{~g}, 0.18-0.88 \mathrm{mmol})$ in $10 \mathrm{~mL}$ of dry THF. Then, reaction was cooled to -78 ${ }^{\circ} \mathrm{C}$ and $1.6 \mathrm{M}$ BuLi solution in hexanes $(0.17-0.83 \mathrm{~mL}, 0.27-1.32 \mathrm{mmol})$ was added and the resulting mixture was stirred at $-78{ }^{\circ} \mathrm{C}$ for $0.5 \mathrm{~h}$. Then, the bath was taken out and electrophile (0.36-1.76 mmol) was added. The mixture was stirred for overnight then was quenched with saturated $\mathrm{NH}_{4} \mathrm{Cl}$ solution and formed mixture was extracted with DCM (3x15 mL). Combined organic phases were dried over anhydrous magnesium sulfate, filtered and evaporated to dryness. The residue was purified by flash chromatography using hexane/EtOAc 2:1 to 50:1 as eluent to give product.

t-Butyl-(4-hydroxy-4-phenyl-but-1-en-1-yl)phenylphosphine-borane (8ag). Mixture of two diastereoisomers, dr 61:39, yield $97 \%$ from $0.193 \mathrm{~g}(0.88 \mathrm{mmol})$ of 1a, purified with hexane/EtOAc (6/1), yellow oil.

Major: ${ }^{1} \mathrm{H} \mathrm{NMR}\left(\mathrm{CDCl}_{3}, 300 \mathrm{MHz}\right): \delta$ 0.11-1.26 (bm, BH $\left.3,3 \mathrm{H}\right), 0.99\left(\mathrm{~d}, J_{\mathrm{P}-\mathrm{H}} 14.0 \mathrm{~Hz}, t-\mathrm{Bu}, 9 \mathrm{H}\right)$, 2.64-2.84 (m, 2H), 2.85 (bs, OH, 1H), 4.79-4.86 (m, 1H), 6.17-6.28 (m, 1H), 6.71-6.90 (m, 1H), 7.29-7.36 (m, 5H), 7.36-7.49 (m, 3H), 7.56-7.70 (m, 2H). ${ }^{13} \mathrm{C} \mathrm{NMR}\left(\mathrm{CDCl}_{3}, 75 \mathrm{MHz}\right): \delta 25.24$ $\left(\mathrm{d}, J_{\mathrm{P}-\mathrm{C}} 2.9 \mathrm{~Hz}\right), 29.32\left(\mathrm{~d}, J_{\mathrm{P}-\mathrm{C}} 35.1 \mathrm{~Hz}\right), 44.86\left(\mathrm{~d}, J_{\mathrm{P}-\mathrm{C}} 14.1 \mathrm{~Hz}\right), 72.90\left(\mathrm{~d}, J_{\mathrm{P}-\mathrm{C}} 1.2 \mathrm{~Hz}\right), 117.50(\mathrm{~d}$, $\left.J_{\mathrm{P}-\mathrm{C}} 54.0 \mathrm{~Hz}\right), 125.83,127.69,128.10\left(\mathrm{~d}, J_{\mathrm{P}-\mathrm{C}} 9.5 \mathrm{~Hz}\right), 128.48,130.77$ (d, $\left.J_{\mathrm{P}-\mathrm{C}} 2.6 \mathrm{~Hz}\right), 133.02(\mathrm{~d}$, $\left.J_{\mathrm{P}-\mathrm{C}} 7.8 \mathrm{~Hz}\right), 143.34,149.95\left(\mathrm{~d}, J_{\mathrm{P}-\mathrm{C}} 9.5 \mathrm{~Hz}\right) .{ }^{31} \mathrm{P} \mathrm{NMR}\left(\mathrm{CDCl}_{3}, 121.5 \mathrm{MHz}\right): \delta 25.59(\mathrm{bm})$.

Minor: ${ }^{1} \mathrm{H} \mathrm{NMR}\left(\mathrm{CDCl}_{3}, 300 \mathrm{MHz}\right): \delta$ 0.11-1.26 (bm, $\left.\mathrm{BH}_{3}, 3 \mathrm{H}\right), 1.04\left(\mathrm{~d}, J_{\mathrm{P}-\mathrm{H}} 14.2 \mathrm{~Hz}, t-\mathrm{Bu}, 9 \mathrm{H}\right)$, 2.64-2.84 (m, 2H), $2.85(\mathrm{bs}, \mathrm{OH}, 1 \mathrm{H}), 4.79-4.86(\mathrm{~m}, 1 \mathrm{H}), 6.17-6.28(\mathrm{~m}, 1 \mathrm{H}), 6.71-6.90(\mathrm{~m}, 1 \mathrm{H})$, 7.29-7.36 (m, 5H), 7.36-7.49 (m, 3H), 7.56-7.70 (m, 2H). ${ }^{13} \mathrm{C} \mathrm{NMR}\left(\mathrm{CDCl}_{3}, 75 \mathrm{MHz}\right): \delta 25.30$ $\left(\mathrm{d}, J_{\mathrm{P}-\mathrm{C}} 2.9 \mathrm{~Hz}\right), 29.35\left(\mathrm{~d}, J_{\mathrm{P}-\mathrm{C}} 35.1 \mathrm{~Hz}\right), 44.83\left(\mathrm{~d}, J_{\mathrm{P}-\mathrm{C}} 14.1 \mathrm{~Hz}\right), 72.85$ (d, $\left.J_{\mathrm{P}-\mathrm{C}} 1.2 \mathrm{~Hz}\right), 117.46(\mathrm{~d}$, $\left.J_{\mathrm{P}-\mathrm{C}} 54.3 \mathrm{~Hz}\right), 125.74,127.64,128.08\left(\mathrm{~d}, J_{\mathrm{P}-\mathrm{C}} 9.5 \mathrm{~Hz}\right), 128.46,130.73$ (d, $\left.J_{\mathrm{P}-\mathrm{C}} 3.2 \mathrm{~Hz}\right), 133.00$ (d, 
$\left.J_{\mathrm{P}-\mathrm{C}} 8.1 \mathrm{~Hz}\right), 143.41,150.03\left(\mathrm{~d}, J_{\mathrm{P}-\mathrm{C}} 8.9 \mathrm{~Hz}\right) .{ }^{31} \mathrm{P} \mathrm{NMR}\left(\mathrm{CDCl}_{3}, 121.5 \mathrm{MHz}\right): \delta 25.59$ (bm). Elem. anal. for $\mathrm{C}_{20} \mathrm{H}_{28} \mathrm{BOP}$ : calc. C 73.64, $\mathrm{H}$ 8.65; found $\mathrm{C} 73.78, \mathrm{H} 8.80$.

(4-Hydroxy-4-phenyl-but-1-en-1-yl)diphenylphosphine-borane (8bg). Yield 77\% from 0.049 $\mathrm{g}(0.20 \mathrm{mmol})$ of $\mathbf{1 b}$, purified with hexane/EtOAc (2:1), yellow oil. ${ }^{1} \mathrm{H} \mathrm{NMR}\left(\mathrm{CDCl}_{3}, 300 \mathrm{MHz}\right)$ : $\delta$ 0.31-1.49 (bm, BH $3,3 \mathrm{H}), 2.08$ (bs, OH, 1H), 2.53-2.76 (m, 2H), 4.70-4.79 (m, 1H), 5.94-6.10 $(\mathrm{m}, 1 \mathrm{H}), 6.36-6.57(\mathrm{~m}, 1 \mathrm{H}), 7.14-7.49(\mathrm{~m}, 15 \mathrm{H}) .{ }^{13} \mathrm{C} \mathrm{NMR}\left(\mathrm{CDCl}_{3}, 75 \mathrm{MHz}\right): \delta 44.40\left(\mathrm{~d}, J_{\mathrm{P}-\mathrm{C}}\right.$ $15.2 \mathrm{~Hz}), 72.93\left(\mathrm{~d}, J_{\mathrm{P}-\mathrm{C}} 1.2 \mathrm{~Hz}\right), 121.85\left(\mathrm{~d}, J_{\mathrm{P}-\mathrm{C}} 57.5 \mathrm{~Hz}\right), 125.80,127.75,128.54,128.63\left(\mathrm{~d}, J_{\mathrm{P}-\mathrm{C}}\right.$ $10.1 \mathrm{~Hz}), 128.66$ (d, J $\left.J_{\mathrm{P}-\mathrm{C}} 10.4 \mathrm{~Hz}\right), 129.59$ (d, $\left.J_{\mathrm{P}-\mathrm{C}} 58.9 \mathrm{~Hz}\right), 129.64$ (d, J $\left.J_{\mathrm{P}-\mathrm{C}} 59.2 \mathrm{~Hz}\right), 130.94$ (d, $\left.J_{\mathrm{P}-\mathrm{C}} 2.6 \mathrm{~Hz}\right), 130.99\left(\mathrm{~d}, J_{\mathrm{P}-\mathrm{C}} 2.3 \mathrm{~Hz}\right), 132.31\left(\mathrm{~d}, J_{\mathrm{P}-\mathrm{C}} 9.5 \mathrm{~Hz}\right), 132.37$ (d, $\left.J_{\mathrm{P}-\mathrm{C}} 9.8 \mathrm{~Hz}\right), 143.25$, $148.15\left(\mathrm{~d}, J_{\mathrm{P}-\mathrm{C}} 7.5 \mathrm{~Hz}\right) .{ }^{31} \mathrm{P} \mathrm{NMR}\left(\mathrm{CDCl}_{3}, 121.5 \mathrm{MHz}\right): \delta 13.55(\mathrm{~m})$. Elem. anal. for $\mathrm{C}_{22} \mathrm{H}_{24} \mathrm{BOP}$ : calc. C 76.32, H 6.99; found C 76.50, H 7.04.

5-(1-Hydroxy-1-phenylmethyl)-2-phospholene-borane (12cg). Yield 41\% from $0.049 \mathrm{~g}(0.28$ mmol) of 1c, purified with hexane/EtOAc (6:1) mixture of two diastereoisomers, dr 59:41, as a mixture with benzyl alcohol (29\%). ${ }^{1} \mathrm{H}-\mathrm{NMR}\left(\mathrm{CDCl}_{3}, 300 \mathrm{MHz}\right): \delta 0.14-1.30\left(\mathrm{bm}, \mathrm{BH}_{3}, 3 \mathrm{H}\right)$, 2.14-2.41 (m, 3H), 3.36-3.48 (bs, OH, 1H), 4.39-4.65 (m, 1H), 5.96-6.11 (m, 1H), 6.02-6.20 (m, $1 \mathrm{H})$, 6.27-6.45 (m, 1H), 6.95-7.11 (m, 1H), 7.19-7.44 (m, 8H), 7.48-7.60 (m, 2H). ${ }^{31} \mathrm{P}$ NMR $\left(\mathrm{CDCl}_{3}, 121.5 \mathrm{MHz}\right): \delta 26.58$ and $28.89(\mathrm{~m})$.

t-Butyl-(4-hydroxy-4-(p-tolyl)-but-1-en-1-yl)phenylphosphine-borane (8ah). Mixture of two diastereoisomers, dr 50:50, yield 64\% from $0.042 \mathrm{~g}(0.19 \mathrm{mmol})$ of 1a, purified with hexane/EtOAc (6/1), colorless oil. ${ }^{1} \mathrm{H}$ NMR $\left(\mathrm{CDCl}_{3}, 300 \mathrm{MHz}\right): \delta$ 0.07-1.18 (bm, $\left.\mathrm{BH}_{3}, 3 \mathrm{H}\right), 0.92$ $\left(\mathrm{d}, J_{\mathrm{P}-\mathrm{H}} 14.0 \mathrm{~Hz}, t\right.$-Bu, 9H) and $0.97\left(\mathrm{~d}, J_{\mathrm{P}-\mathrm{H}}=14.2 \mathrm{~Hz}, t-\mathrm{Bu}, 9 \mathrm{H}\right), 2.03(\mathrm{bs}, \mathrm{OH}, 1 \mathrm{H}), 2.23(\mathrm{~s}, 3 \mathrm{H})$, 2.54-2.73 (m, 2H), 4.95-5.03 (m, 1H), 6.09-6.22 (m, 1H), 6.66-6.86 (m, 1H), 6.95-7.18 (m, 4H), 7.27-7.42 (m, 3H), 7.56-7.64 (m, 2H) and 7.48-7.56 (m, 2H). ${ }^{13} \mathrm{C} \mathrm{NMR}\left(\mathrm{CDCl}_{3}, 75 \mathrm{MHz}\right): \delta$ 21.55 and 21.57, $25.75\left(\mathrm{~d}, J_{\mathrm{P}-\mathrm{C}} 2.9 \mathrm{~Hz}\right)$ and $2.83\left(\mathrm{~d}, J_{\mathrm{P}-\mathrm{C}} 2.6 \mathrm{~Hz}\right), 29.85\left(\mathrm{~d}, J_{\mathrm{P}-\mathrm{C}} 35.1 \mathrm{~Hz}\right)$ and 29.9 $\left(\mathrm{d}, J_{\mathrm{P}-\mathrm{C}} 35.1 \mathrm{~Hz}\right), 45.30\left(\mathrm{~d}, J_{\mathrm{P}-\mathrm{C}} 14.1 \mathrm{~Hz}\right), 73.26\left(\mathrm{~d}, J_{\mathrm{P}-\mathrm{C}} 1.2 \mathrm{~Hz}\right)$ and $73.29\left(\mathrm{~d}, J_{\mathrm{P}-\mathrm{C}} 1.2 \mathrm{~Hz}\right), 117.86$ $\left(\mathrm{d}, J_{\mathrm{P}-\mathrm{C}} 54.0 \mathrm{~Hz}\right)$ and $117.90\left(\mathrm{~d}, J_{\mathrm{P}-\mathrm{C}} 54.3 \mathrm{~Hz}\right), 126.22$ and $126.31,128.54\left(\mathrm{~d}, J_{\mathrm{P}-\mathrm{C}} 52.3 \mathrm{~Hz}\right)$ and $128.60\left(\mathrm{~d}, J_{\mathrm{P}-\mathrm{C}} 52.3 \mathrm{~Hz}\right), 128.57\left(\mathrm{~d}, J_{\mathrm{P}-\mathrm{C}} 8.9 \mathrm{~Hz}\right)$ and $128.62\left(\mathrm{~d}, J_{\mathrm{P}-\mathrm{C}} 9.3 \mathrm{~Hz}\right), 129.64$ and 129.65, $131.24\left(\mathrm{~d}, J_{\mathrm{P}-\mathrm{C}} 2.3 \mathrm{~Hz}\right)$ and $131.30\left(\mathrm{~d}, J_{\mathrm{P}-\mathrm{C}} 2.6 \mathrm{~Hz}\right), 133.54\left(\mathrm{~d}, J_{\mathrm{P}-\mathrm{C}} 7.8 \mathrm{~Hz}\right)$ and $133.55\left(\mathrm{~d}, J_{\mathrm{P}-\mathrm{C}} 7.8\right.$ $\mathrm{Hz}), 137.73$ and $138.36,150.61\left(\mathrm{~d}, J_{\mathrm{P}-\mathrm{C}} 9.2 \mathrm{~Hz}\right)$ and $150.70\left(\mathrm{~d}, J_{\mathrm{P}-\mathrm{C}} 9.2 \mathrm{~Hz}\right) .{ }^{31} \mathrm{P} \mathrm{NMR}\left(\mathrm{CDCl}_{3}\right.$, $121.5 \mathrm{MHz}): \delta 25.43(\mathrm{~m})$. Elem. anal. for $\mathrm{C}_{21} \mathrm{H}_{30}$ BOP: calc. C 74.13, $\mathrm{H}$ 8.89; found $\mathrm{C} 74.00, \mathrm{H}$ 8.70 .

(4-Hydroxy-4-(p-tolil)-but-1-en-1-yl)diphenylphosphine-borane (8bh). Yield 65\% from 0.066 $\mathrm{g}(0.28 \mathrm{mmol})$ of $\mathbf{1 b}$, purified with hexane/EtOAc (6:1), yellow oil. ${ }^{1} \mathrm{H} \mathrm{NMR}\left(\mathrm{CDCl}_{3}, 300 \mathrm{MHz}\right)$ : $\delta$ 0.24-1.63 (m, BH $3,3 \mathrm{H}), 1.90$ (bs, OH, 1H), 2.26 (s, 3H), 2.48-2.75 (m, 2H), 4.69-4.76 (m, 1H), 5.95-6.08 (m, 1H), 6.38-6.58 (m, 1H), 7.01-7.18 (m, 4H), 7.23-7.50 (m, 10H). ${ }^{13} \mathrm{C} \mathrm{NMR}\left(\mathrm{CDCl}_{3}\right.$, $75 \mathrm{MHz}): \delta 21.10,44.34\left(\mathrm{~d}, J_{\mathrm{P}-\mathrm{C}} 14.9 \mathrm{~Hz}\right), 72.78\left(\mathrm{~d}, J_{\mathrm{P}-\mathrm{C}} 0.9 \mathrm{~Hz}\right), 121.58\left(\mathrm{~d}, J_{\mathrm{P}-\mathrm{C}} 57.2 \mathrm{~Hz}\right)$, $125.75,128.59$ (d, JP-C $10.4 \mathrm{~Hz}), 128.63$ (d, J $\left.J_{\mathrm{P}-\mathrm{C}} 10.1 \mathrm{~Hz}\right), 129.17,129.63$ (d, $\left.J_{\mathrm{P}-\mathrm{C}} 58.9 \mathrm{~Hz}\right)$, $129.70\left(\mathrm{~d}, J_{\mathrm{P}-\mathrm{C}} 58.9 \mathrm{~Hz}\right), 130.90\left(\mathrm{~d}, J_{\mathrm{P}-\mathrm{C}} 2.6 \mathrm{~Hz}\right), 130.97\left(\mathrm{~d} J_{\mathrm{P}-\mathrm{C}} 2.9 \mathrm{~Hz}\right), 132.31$ (d, $\left.J_{\mathrm{P}-\mathrm{C}} 9.5 \mathrm{~Hz}\right)$, $132.37\left(\mathrm{~d}, J_{\mathrm{P}-\mathrm{C}} 9.5 \mathrm{~Hz}\right), 147.40,140.26,148.33$ (d, $J_{\mathrm{P}-\mathrm{C}} 7.5 \mathrm{~Hz} .{ }^{31} \mathrm{P}$ NMR $\left(\mathrm{CDCl}_{3}, 121.5 \mathrm{MHz}\right): \delta$ 13.56 (m). Elem. anal. for $\mathrm{C}_{23} \mathrm{H}_{26}$ BOP: calc. C 76.68, H 7.27; found C 76.84, H 7.20. 
t-Butyl-(4-hydroxy-4-(o-tolyl)-but-1-en-1-yl)phenylphosphine-borane (8ai). Mixture of two diastereoisomers, dr 53:47, yield $67 \%$ from $0.065 \mathrm{~g}(0.29 \mathrm{mmol})$ of 1a, purified with hexane/EtOAc (6/1), pale yellow oil.

Major: ${ }^{1} \mathrm{H}$ NMR $\left(\mathrm{CDCl}_{3}, 300 \mathrm{MHz}\right): \delta$ 0.06-1.11 (bm, $\left.\mathrm{BH}_{3}, 3 \mathrm{H}\right), 0.92\left(\mathrm{~d}, J_{\mathrm{P}-\mathrm{H}} 14.2 \mathrm{~Hz}, t\right.$-Bu, $9 \mathrm{H}), 1.86$ (bs, OH, 1H), $2.23(\mathrm{~s}, 3 \mathrm{H}), 2.58-2.68(\mathrm{~m}, 2 \mathrm{H}), 4.96-5.02(\mathrm{~m}, 1 \mathrm{H}), 6.10-6.22(\mathrm{~m}, 1 \mathrm{H})$, 6.67-6.86 (m, 1H), 6.95-7.18 (m, 4H), 7.27-7.42 (m, 3H), 7.56-7.64 (m, 2H). ${ }^{13} \mathrm{C} \mathrm{NMR}\left(\mathrm{CDCl}_{3}\right.$,

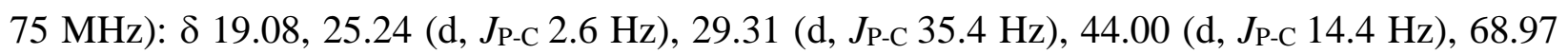
$\left(\mathrm{d}, J_{\mathrm{P}-\mathrm{C}} 1.4 \mathrm{~Hz}\right), 117.52\left(\mathrm{~d}, J_{\mathrm{P}-\mathrm{C}} 54.0 \mathrm{~Hz}\right), 125.23,127.40,128.08\left(\mathrm{~d}, J_{\mathrm{P}-\mathrm{C}} 52.0 \mathrm{~Hz}\right), 128.12\left(\mathrm{~d}, J_{\mathrm{P}-\mathrm{C}}\right.$ $9.5 \mathrm{~Hz}), 128.14,130.43,130.78$ (d, $\left.J_{\mathrm{P}-\mathrm{C}} 2.6 \mathrm{~Hz}\right), 133.00$ (d, $\left.J_{\mathrm{P}-\mathrm{C}} 7.8 \mathrm{~Hz}\right), 134.33,141.33,150.06$ $\left(\mathrm{d}, J_{\mathrm{P}-\mathrm{C}} 9.5 \mathrm{~Hz}\right) .{ }^{31} \mathrm{P}$ NMR $\left(\mathrm{CDCl}_{3}, 121.5 \mathrm{MHz}\right): \delta 25.44(\mathrm{~m})$.

Minor: ${ }^{1} \mathrm{H}$ NMR $\left(\mathrm{CDCl}_{3}, 300 \mathrm{MHz}\right): \delta$ 0.06-1.11 (bm, BH $\left.3,3 \mathrm{H}\right), 0.98\left(\mathrm{~d}, J_{\mathrm{P}-\mathrm{H}} 14.1 \mathrm{~Hz}, t-\mathrm{Bu}, 9 \mathrm{H}\right)$, $1.86(\mathrm{bs}, \mathrm{OH}, 1 \mathrm{H}), 2.17(\mathrm{~s}, 3 \mathrm{H}), 2.58-2.68(\mathrm{~m}, 2 \mathrm{H}), 4.96-5.02(\mathrm{~m}, 1 \mathrm{H}), 6.10-6.22(\mathrm{~m}, 1 \mathrm{H}), 6.67-$ $6.86(\mathrm{~m}, 1 \mathrm{H}), 6.95-7.18(\mathrm{~m}, 4 \mathrm{H}), 7.27-7.42(\mathrm{~m}, 3 \mathrm{H}), 7.48-7.56(\mathrm{~m}, 2 \mathrm{H}) .{ }^{13} \mathrm{C} \mathrm{NMR}\left(\mathrm{CDCl}_{3}, 75\right.$ MHz): $\delta$ 19.01, 25.32 (d, $\left.J_{\mathrm{P}-\mathrm{C}} 2.9 \mathrm{~Hz}\right), 29.37$ (d, $\left.J_{\mathrm{P}-\mathrm{C}} 35.1 \mathrm{~Hz}\right), 43.96$ (d, $\left.J_{\mathrm{P}-\mathrm{C}} 14.1 \mathrm{~Hz}\right), 68.97$ (d, $\left.J_{\mathrm{P}-\mathrm{C}} 1.4 \mathrm{~Hz}\right), 117.36\left(\mathrm{~d}, J_{\mathrm{P}-\mathrm{C}} 54.0 \mathrm{~Hz}\right), 125.17,127.35,128.00\left(\mathrm{~d}, J_{\mathrm{P}-\mathrm{C}} 52.3 \mathrm{~Hz}\right), 128.22\left(\mathrm{~d}, J_{\mathrm{P}-\mathrm{C}} 9.5\right.$ $\mathrm{Hz}), 128.94,130.41,130.73$ (d, J $\left.J_{\mathrm{P}-\mathrm{C}} 2.6 \mathrm{~Hz}\right), 132.96$ (d, $\left.J_{\mathrm{P}-\mathrm{C}} 8.1 \mathrm{~Hz}\right), 134.29,141.45,150.15$ (d, $\left.J_{\mathrm{P}-\mathrm{C}} 9.2 \mathrm{~Hz}\right) .{ }^{31} \mathrm{P} \mathrm{NMR}\left(\mathrm{CDCl}_{3}, 121.5 \mathrm{MHz}\right): \delta 25.44(\mathrm{~m})$. Elem. anal. for $\mathrm{C}_{21} \mathrm{H}_{30} \mathrm{BOP}$ : calc. C 74.13, H 8.89; found C 74.38, H 8.99.

(4-Hydroxy-4-(o-tolil)-but-1-en-1-yl)diphenylphosphine-borane (8bi). Yield 68\% from 0.066 $\mathrm{g}(0.27 \mathrm{mmol})$ of $\mathbf{1 b}$, purified with hexane/EtOAc (6:1), yellow oil. ${ }^{1} \mathrm{H} \mathrm{NMR}\left(\mathrm{CDCl}_{3}, 300 \mathrm{MHz}\right)$ : $\delta$ 0.32-1.61 (bm, $\left.\mathrm{BH}_{3}, 3 \mathrm{H}\right), 1.75(\mathrm{bs}, 1 \mathrm{H}, \mathrm{OH}), 2.22(\mathrm{~s}, 3 \mathrm{H}), 2.57-2.73(\mathrm{~m}, 2 \mathrm{H}), 4.99-5.08(\mathrm{~m}$, $1 \mathrm{H})$, 5.98-6.16 (m, 1H), 6.43-6.62 (m, 1H), 7.00-7.20 (m, 4H), 7.24-7.52 (bm, 10H). ${ }^{13} \mathrm{C} \mathrm{NMR}$ $\left(\mathrm{CDCl}_{3}, 75 \mathrm{MHz}\right): \delta 19.12,43.49\left(\mathrm{~d}, J_{\mathrm{P}-\mathrm{C}} 15.2 \mathrm{~Hz}\right), 69.12\left(\mathrm{~d}, J_{\mathrm{P}-\mathrm{C}} 1.2 \mathrm{~Hz}\right), 121.87$ (d, $J_{\mathrm{P}-\mathrm{C}} 57.2$ $\mathrm{Hz}), 125.29,126.42,127.48,128.67\left(\mathrm{~d}, J_{\mathrm{P}-\mathrm{C}} 10.4 \mathrm{~Hz}\right), 128.70\left(\mathrm{~d}, J_{\mathrm{P}-\mathrm{C}} 10.1 \mathrm{~Hz}\right), 129.68\left(\mathrm{~d}, J_{\mathrm{P}-\mathrm{C}}\right.$ $59.2 \mathrm{~Hz}), 129.70\left(\mathrm{~d}, J_{\mathrm{P}-\mathrm{C}} 58.9 \mathrm{~Hz}\right), 130.48,130.96\left(\mathrm{~d}, J_{\mathrm{P}-\mathrm{C}} 2.6 \mathrm{~Hz}\right), 131.01$ (d, $\left.J_{\mathrm{P}-\mathrm{C}} 2.3 \mathrm{~Hz}\right), 132.33$ $\left(\mathrm{d}, J_{\mathrm{P}-\mathrm{C}} 9.5 \mathrm{~Hz}\right), 132.39\left(\mathrm{~d}, J_{\mathrm{P}-\mathrm{C}} 9.5 \mathrm{~Hz}\right), 134.36,141.34,148.25$ (d, $\left.J_{\mathrm{P}-\mathrm{C}} 7.5 \mathrm{~Hz}\right) .{ }^{31} \mathrm{P}$ NMR $\left(\mathrm{CDCl}_{3}, 121.5 \mathrm{MHz}\right): \delta 13.37(\mathrm{~m})$. Elem. anal. for $\mathrm{C}_{23} \mathrm{H}_{26} \mathrm{BOP}$ : calc. C 76.68, H 7.27; found C 76.70, H 7.25.

t-Butyl-(4-hydroxy-4-methylpent-1-en-1-yl)phenylphosphine-borane (8aj). Yield 99\% from $0.045 \mathrm{~g}(0.2 \mathrm{mmol})$ of 1a, purified with hexane/EtOAc (1/1), pale yellow oil. ${ }^{1} \mathrm{H} \mathrm{NMR}\left(\mathrm{CDCl}_{3}\right.$, $300 \mathrm{MHz}$ ): $\delta$ 0.11-1.20 (bm, BH $3,3 \mathrm{H}), 1.30$ (d, $\left.J_{\mathrm{P}-\mathrm{H}} 14.2 \mathrm{~Hz}, t-\mathrm{Bu}, 9 \mathrm{H}\right), 1.18(\mathrm{~s}, 6 \mathrm{H}), 1.77$ (bs, $\mathrm{OH}, 1 \mathrm{H}), 2.39-2.44(\mathrm{~m}, 2 \mathrm{H}), 6.20-6.30(\mathrm{~m}, 1 \mathrm{H}), 6.73-6.90(\mathrm{~m}, 1 \mathrm{H}), 7.31-7.41(\mathrm{~m}, 3 \mathrm{H}), 7.62-7.70$ $(\mathrm{m}, 2 \mathrm{H}) .{ }^{13} \mathrm{C} \mathrm{NMR}\left(\mathrm{CDCl}_{3}, 75 \mathrm{MHz}\right): \delta 25.30\left(\mathrm{~d}, J_{\mathrm{P}-\mathrm{C}} 2.6 \mathrm{~Hz}\right), 29.16,29.25,29.35\left(\mathrm{~d}, J_{\mathrm{P}-\mathrm{C}} 35.1\right.$ $\mathrm{Hz}), 49.52$ (d, $\left.J_{\mathrm{P}-\mathrm{C}} 14.4 \mathrm{~Hz}\right), 70.57\left(\mathrm{~d}, J_{\mathrm{P}-\mathrm{C}} 1.2 \mathrm{~Hz}\right), 117.68$ (d, $\left.J_{\mathrm{P}-\mathrm{C}} 54.0 \mathrm{~Hz}\right) .128 .06$ (d, $J_{\mathrm{P}-\mathrm{C}} 52.3$ $\mathrm{Hz}), 128.11\left(\mathrm{~d}, J_{\mathrm{P}-\mathrm{C}} 9.5 \mathrm{~Hz}\right), 130.80\left(\mathrm{~d}, J_{\mathrm{P}-\mathrm{C}} 2.6 \mathrm{~Hz}\right), 132.98$ (d, $\left.J_{\mathrm{P}-\mathrm{C}} 8.1 \mathrm{~Hz}\right), 150.51$ (d, J $J_{\mathrm{P}-\mathrm{C}} 8.9$ $\mathrm{Hz}) .{ }^{31} \mathrm{P} \mathrm{NMR}\left(\mathrm{CDCl}_{3}, 121.5 \mathrm{MHz}\right): \delta 25.43(\mathrm{~m})$. Elem. anal. for $\mathrm{C}_{16} \mathrm{H}_{28} \mathrm{BOP}$ : calc. C 69.08, $\mathrm{H}$ 10.15; found C 69.00, H 9.99.

(4-Hydroxy-4-methylpent-1-en-1-yl)diphenylphosphine-borane (8bj). Yield $73 \%$ from 0.046 $\mathrm{g}(0.19 \mathrm{mmol})$ of $\mathbf{1 b}$, purified with hexane/EtOAc (6:1), yellow oil. ${ }^{1} \mathrm{H} \mathrm{NMR}\left(\mathrm{CDCl}_{3}, 300 \mathrm{MHz}\right)$ : $\delta$ 0.33-1.62 (bm, $\left.\mathrm{BH}_{3}, 3 \mathrm{H}\right), 1.16(\mathrm{~s}, 6 \mathrm{H}), 1.82$ (bs, OH, 1H), 2.35-2.41 (m, 2H), 6.09-6.21 (m, 
1H), 6.50-6.69 (m, 1H), 7.29-7.43 (m, 6H), 7.50-7.59 (m, 4H). ${ }^{13} \mathrm{C} \mathrm{NMR}\left(\mathrm{CDCl}_{3}, 75 \mathrm{MHz}\right): \delta$ 29.34, $49.11\left(\mathrm{~d}, J_{\mathrm{P}-\mathrm{C}} 14.1 \mathrm{~Hz}\right), 70.66$ (d, $\left.J_{\mathrm{P}-\mathrm{C}} 1.2 \mathrm{~Hz}\right), 122.18$ (d, $\left.J_{\mathrm{P}-\mathrm{C}} 57.2 \mathrm{~Hz}\right), 128.73$ (d, $J_{\mathrm{P}-\mathrm{C}} 10.4$ $\mathrm{Hz}), 129.82$ (d, $\left.J_{\mathrm{P}-\mathrm{C}} 58.9 \mathrm{~Hz}\right), 131.04\left(\mathrm{~d}, J_{\mathrm{P}-\mathrm{C}} 2.6 \mathrm{~Hz}\right), 132.43\left(\mathrm{~d}, J_{\mathrm{P}-\mathrm{C}} 9.5 \mathrm{~Hz}\right), 148.70$ (d, J $J_{\mathrm{P}-\mathrm{C}} 7.5$ $\mathrm{Hz}) .{ }^{31} \mathrm{P}$ NMR $\left(\mathrm{CDCl}_{3}, 121.5 \mathrm{MHz}\right): \delta 13.36(\mathrm{~m})$. Elem. anal. for $\mathrm{C}_{18} \mathrm{H}_{24} \mathrm{BOP}$ : calc. C 72.51, $\mathrm{H}$ 8.11; found C 72.74, H 7.99.

t-Butyl-(4-cyclohexylidene-4-hydroxy-but-1-en-1-yl)phenylphosphine-borane (8ak). Yield $71 \%$ from $0.047 \mathrm{~g}(0.21 \mathrm{mmol})$ of 1a, purified with hexane/EtOAc $(6 / 1)$, colorless oil. ${ }^{1} \mathrm{H}$ NMR $\left(\mathrm{CDCl}_{3}, 300 \mathrm{MHz}\right): \delta$ 0.08-1.29 (bm, BH, $\left.3 \mathrm{H}\right), 1.02\left(\mathrm{~d}, J_{\mathrm{P}-\mathrm{H}} 14.1 \mathrm{~Hz}, t-\mathrm{Bu}, 9 \mathrm{H}\right), 1.30-1.59(\mathrm{~m}$, $10 \mathrm{H}), 2.37-2.43(\mathrm{~m}, 2 \mathrm{H}), 6.19-6.30(\mathrm{~m}, 1 \mathrm{H}), 6.75-6.92(\mathrm{~m}, 1 \mathrm{H}), 7.31-7.44(\mathrm{~m}, 3 \mathrm{H}), 7.62-7.70(\mathrm{~m}$, 2H). ${ }^{13} \mathrm{C} \mathrm{NMR}\left(\mathrm{CDCl}_{3}, 75 \mathrm{MHz}\right): \delta 21.99,22.01,25.37\left(\mathrm{~d}, J_{\mathrm{P}-\mathrm{C}} 2.9 \mathrm{~Hz}\right), 25.51,29.40\left(\mathrm{~d}, J_{\mathrm{P}-\mathrm{C}}\right.$ $35.1 \mathrm{~Hz}), 37.41,37.50,48.35\left(\mathrm{~d}, J_{\mathrm{P}-\mathrm{C}} 13.8 \mathrm{~Hz}\right), 71.44$ (d, $\left.J_{\mathrm{P}-\mathrm{C}} 0.9 \mathrm{~Hz}\right), 117.81$ (d, $\left.J_{\mathrm{P}-\mathrm{C}} 53.7 \mathrm{~Hz}\right)$, $128.16\left(\mathrm{~d}, J_{\mathrm{P}-\mathrm{C}} 9.2 \mathrm{~Hz}\right), 128.19\left(\mathrm{~d}, J_{\mathrm{P}-\mathrm{C}} 51.7 \mathrm{~Hz}\right), 130.83\left(\mathrm{~d}, J_{\mathrm{P}-\mathrm{C}} 2.6 \mathrm{~Hz}\right), 133.04\left(\mathrm{~d}, J_{\mathrm{P}-\mathrm{C}} 7.8 \mathrm{~Hz}\right)$, $150.21\left(\mathrm{~d}, J_{\mathrm{P}-\mathrm{C}} 9.5 \mathrm{~Hz}\right) .{ }^{31} \mathrm{P} \mathrm{NMR}\left(\mathrm{CDCl}_{3}, 121.5 \mathrm{MHz}\right): \delta 25.32(\mathrm{~m})$. Elem. anal. for $\mathrm{C}_{19} \mathrm{H}_{32} \mathrm{BOP}$ : calc. C 71.71, H 10.14; found C 71.79, H 9.97.

(4-Cyclohexylidene-4-hydroxy-but-1-en-1-yl)diphenylphosphine-borane (8bk). Yield 53\% from $0.038 \mathrm{~g}(0.16 \mathrm{mmol})$ of $\mathbf{1 b}$, purified with hexane/EtOAc (6:1), colorless oil. ${ }^{1} \mathrm{H}$ NMR $\left(\mathrm{CDCl}_{3}, 300 \mathrm{MHz}\right): \delta$ 0.30-1.48 (bm, $\left.\mathrm{BH}_{3}, 3 \mathrm{H}\right), 1.27-1.58(\mathrm{~m}, 10 \mathrm{H}), 2.34-2.41(\mathrm{~m}, 2 \mathrm{H}), 6.08-$ $6.21(\mathrm{~m}, 1 \mathrm{H}), 6.54-6.73(\mathrm{~m}, 1 \mathrm{H}), 7.30-7.44(\mathrm{~m}, 6 \mathrm{H}), 7.49-7.60(\mathrm{~m}, 4 \mathrm{H}) .{ }^{13} \mathrm{C} \mathrm{NMR}\left(\mathrm{CDCl}_{3}, 75\right.$ MHz): $\delta$ 22.02, 25.50, 37.52, 47.99 (d, JP-C $15.5 \mathrm{~Hz}), 71.45,122.09$ (d, J $\left.J_{\mathrm{P}-\mathrm{C}} 56.9 \mathrm{~Hz}\right), 128.74(\mathrm{~d}$, $\left.J_{\mathrm{P}-\mathrm{C}} 10.1 \mathrm{~Hz}\right), 131.01\left(\mathrm{~d}, J_{\mathrm{P}-\mathrm{C}} 2.6 \mathrm{~Hz}\right), 132.34\left(\mathrm{~d}, J_{\mathrm{P}-\mathrm{C}} 9.5 \mathrm{~Hz}\right), 148.50\left(\mathrm{~d}, J_{\mathrm{P}-\mathrm{C}} 7.8 \mathrm{~Hz}\right) .{ }^{31} \mathrm{P}$ NMR $\left(\mathrm{CDCl}_{3}, 121.5 \mathrm{MHz}\right): \delta 13.29(\mathrm{~m})$. Elem. anal. for $\mathrm{C}_{21} \mathrm{H}_{28} \mathrm{BOP}$ : calc. C 74.57, $\mathrm{H}$ 8.34; found C 74.59, H 8.40.

5-(1'-Hydroxycyclohexyl)-3-phospholene-borane (11ck). Yield $31 \%$ from $0.050 \mathrm{~g}(0.28$ mmol) of 1c, purified with hexane/EtOAc (6:1), colorless oil. ${ }^{1} \mathrm{H}$ NMR $\left(\mathrm{CDCl}_{3}, 300 \mathrm{MHz}\right): \delta$ 0.39-1.76 (bm, $\left.\mathrm{BH}_{3}, 3 \mathrm{H}\right), 1.13-1.68(\mathrm{~m}, 10 \mathrm{H}), 1.77-1.89(\mathrm{~m}, 2 \mathrm{H}), 2.68$ (bs, OH, 1H), 3.08-3.18 $(\mathrm{m}, 1 \mathrm{H}), 5.89-6.12(\mathrm{~m}, 2 \mathrm{H}), 7.31-7.48(\mathrm{~m}, 3 \mathrm{H}), 7.61-7.74(\mathrm{~m}, 2 \mathrm{H}) .{ }^{13} \mathrm{C} \mathrm{NMR}\left(\mathrm{CDCl}_{3}, 75 \mathrm{MHz}\right)$ : $\delta 21.44,21.71,25.43,32.56\left(\mathrm{~d}, J_{\mathrm{P}-\mathrm{C}} 38.2 \mathrm{~Hz}\right), 35.16\left(\mathrm{~d}, J_{\mathrm{P}-\mathrm{C}} 4.6 \mathrm{~Hz}\right), 37.39$ (d, J $\left.J_{\mathrm{P}-\mathrm{C}} 5.5 \mathrm{~Hz}\right), 74.19$ $\left(\mathrm{d}, J_{\mathrm{P}-\mathrm{C}} 2.6 \mathrm{~Hz}\right), 128.29$ (d, $\left.J_{\mathrm{P}-\mathrm{C}} 9.5 \mathrm{~Hz}\right), 129.76,130.41$ (d, $\left.J_{\mathrm{P}-\mathrm{C}} 47.1 \mathrm{~Hz}\right), 131.06$ (d, $\left.J_{\mathrm{P}-\mathrm{C}} 9.2 \mathrm{~Hz}\right)$, $131.32\left(\mathrm{~d}, J_{\mathrm{P}-\mathrm{C}} 11.5 \mathrm{~Hz}\right), 131.41\left(\mathrm{~d}, J_{\mathrm{P}-\mathrm{C}} 2.3 \mathrm{~Hz}\right) .{ }^{31} \mathrm{P} \mathrm{NMR}\left(\mathrm{CDCl}_{3}, 121.5 \mathrm{MHz}\right): \delta 25.38(\mathrm{~m})$. Elem. anal. for $\mathrm{C}_{16} \mathrm{H}_{24}$ BOP: calc. C 70.10, H 8.82; found C 70.35, H 8.90.

t-Butyl-(4-hydroxy-4-phenylpent-1-en-1-yl)phenylphosphine-borane (8al). Mixture of two diastereoisomers, dr 56:44, yield $36 \%$ from $0.084 \mathrm{~g}(0.38 \mathrm{mmol})$ of 1a, purified with hexane/EtOAc (6/1), colorless oil.

Major: ${ }^{1} \mathrm{H} \mathrm{NMR}\left(\mathrm{CDCl}_{3}, 300 \mathrm{MHz}\right): \delta$ 0.05-1.23 (bm, $\left.\mathrm{BH}_{3}, 3 \mathrm{H}\right), 0.90\left(\mathrm{~d}, J_{\mathrm{P}-\mathrm{H}} 14.2 \mathrm{~Hz}, t-\mathrm{Bu}, 9 \mathrm{H}\right)$, $1.53(\mathrm{~s}, 3 \mathrm{H}), 1.93(\mathrm{bs}, \mathrm{OH}, 1 \mathrm{H}), 2.68-2.75(\mathrm{~m}, 2 \mathrm{H}), 6.03-6.13(\mathrm{~m}, 1 \mathrm{H}), 6.56-6.74(\mathrm{~m}, 1 \mathrm{H}), 7.11-$ $7.41(\mathrm{~m}, 8 \mathrm{H}), 7.47-7.57(\mathrm{~m}, 2 \mathrm{H}) .{ }^{13} \mathrm{C} \mathrm{NMR}\left(\mathrm{CDCl}_{3}, 75 \mathrm{MHz}\right): \delta 25.28\left(\mathrm{~d}, J_{\mathrm{P}-\mathrm{C}} 3.2 \mathrm{~Hz}\right), 29.32(\mathrm{~d}$, $\left.J_{\mathrm{P}-\mathrm{C}} 35.3 \mathrm{~Hz}\right), 29.80,49.95\left(\mathrm{~d}, J_{\mathrm{P}-\mathrm{C}} 14.1 \mathrm{~Hz}\right), 74.26\left(\mathrm{~d}, J_{\mathrm{P}-\mathrm{C}} 1.1 \mathrm{~Hz}\right), 118.32\left(\mathrm{~d}, J_{\mathrm{P}-\mathrm{C}} 54.0 \mathrm{~Hz}\right)$, 124.65, 126.80, 127.95 (d, $\left.J_{\mathrm{P}-\mathrm{C}} 52.0 \mathrm{~Hz}\right), 128.07$ (d, $\left.J_{\mathrm{P}-\mathrm{C}} 9.5 \mathrm{~Hz}\right), 128.23,130.73$ (d, $\left.J_{\mathrm{P}-\mathrm{C}} 2.3 \mathrm{~Hz}\right)$, $133.00\left(\mathrm{~d}, J_{\mathrm{P}-\mathrm{C}} 7.8 \mathrm{~Hz}\right), 146.71,149.42\left(\mathrm{~d}, J_{\mathrm{P}-\mathrm{C}} 8.9 \mathrm{~Hz}\right) .{ }^{31} \mathrm{P} \mathrm{NMR}\left(\mathrm{CDCl}_{3}, 121.5 \mathrm{MHz}\right): \delta 25.74$ (m). 
Minor: ${ }^{1} \mathrm{H} \mathrm{NMR}\left(\mathrm{CDCl}_{3}, 300 \mathrm{MHz}\right): \delta$ 0.05-1.23 (bm, BH $\left.3,3 \mathrm{H}\right), 0.94\left(\mathrm{~d}, J_{\mathrm{P}-\mathrm{H}} 14.0 \mathrm{~Hz}, t-\mathrm{Bu}, 9 \mathrm{H}\right)$, 1.54 (s, 3H), 1.93 (bs, OH, 1H), 2.68-2.75 (m, 2H), 6.03-6.13 (m, 1H), 6.56-6.74 (m, 1H), 7.11$7.41(\mathrm{~m}, 8 \mathrm{H}), 7.47-7.57(\mathrm{~m}, 2 \mathrm{H}) .{ }^{13} \mathrm{C} \mathrm{NMR}\left(\mathrm{CDCl}_{3}, 75 \mathrm{MHz}\right): \delta 25.24\left(\mathrm{~d}, J_{\mathrm{P}-\mathrm{C}} 2.9 \mathrm{~Hz}\right), 29.28(\mathrm{~d}$, $\left.J_{\mathrm{P}-\mathrm{C}} 35.1 \mathrm{~Hz}\right), 29.59,49.98\left(\mathrm{~d}, J_{\mathrm{P}-\mathrm{C}} 14.1 \mathrm{~Hz}\right), 74.20\left(\mathrm{~d}, J_{\mathrm{P}-\mathrm{C}} 1.1 \mathrm{~Hz}\right), 118.41\left(\mathrm{~d}, J_{\mathrm{P}-\mathrm{C}} 54.0 \mathrm{~Hz}\right)$, 124.67, 126.85, 127.95 (d, $\left.J_{\mathrm{P}-\mathrm{C}} 52.0 \mathrm{~Hz}\right), 128.07$ (d, $\left.J_{\mathrm{P}-\mathrm{C}} 9.5 \mathrm{~Hz}\right), 128.23,130.73$ (d, $\left.J_{\mathrm{P}-\mathrm{C}} 2.3 \mathrm{~Hz}\right)$, $133.04\left(\mathrm{~d}, J_{\mathrm{P}-\mathrm{C}} 7.8 \mathrm{~Hz}\right), 146.76,149.42\left(\mathrm{~d}, J_{\mathrm{P}-\mathrm{C}} 8.9 \mathrm{~Hz}\right) .{ }^{31} \mathrm{P} \mathrm{NMR}\left(\mathrm{CDCl}_{3}, 121.5 \mathrm{MHz}\right): \delta 25.74$ (m). Elem. anal. for $\mathrm{C}_{21} \mathrm{H}_{30} \mathrm{BOP}$ : calc. C 74.13, $\mathrm{H}$ 8.89; found C 74.01, H 8.70.

t-Butyl-(4-hydroxy-4-phenylpent-2-en-1-yl)phenylphosphine-borane (9al). Mixture of two diastereoisomers, dr 62:38, yield $24 \%$ from $0.084 \mathrm{~g}(0.38 \mathrm{mmol})$ of 1a, purified with hexane/EtOAc (6/1), colorless oil.

Major: ${ }^{1} \mathrm{H} \mathrm{NMR}\left(\mathrm{CDCl}_{3}, 300 \mathrm{MHz}\right): \delta$ 0.07-1.24 (bm, $\left.\mathrm{BH}_{3}, 3 \mathrm{H}\right), 1.05\left(\mathrm{~d}, J_{\mathrm{P}-\mathrm{H}} 13.7 \mathrm{~Hz}, t-\mathrm{Bu}, 9 \mathrm{H}\right)$, $1.40(\mathrm{~s}, 3 \mathrm{H}), 1.69(\mathrm{bs}, \mathrm{OH}, 1 \mathrm{H}), 2.64-2.76(\mathrm{~m}, 1 \mathrm{H}), 2.82-2.96(\mathrm{~m}, 1 \mathrm{H}), 5.52-5.83(\mathrm{~m}, 2 \mathrm{H}), 7.01-$

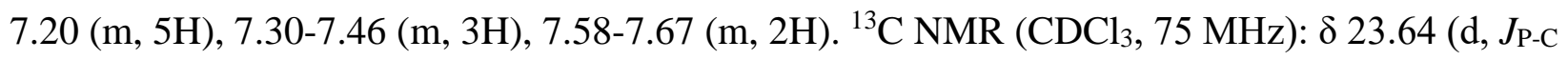
$32.5 \mathrm{~Hz}), 25.49$ (d, $\left.J_{\mathrm{P}-\mathrm{C}} 2.0 \mathrm{~Hz}\right), 29.20,74.32$ (d, $\left.J_{\mathrm{P}-\mathrm{C}} 1.7 \mathrm{~Hz}\right), 119.49$ (d, $\left.J_{\mathrm{P}-\mathrm{C}} 54.0 \mathrm{~Hz}\right), 124.88$, 126.63, 127.98, 128.19 (d, $\left.J_{\mathrm{P}-\mathrm{C}} 9.8 \mathrm{~Hz}\right), 131.13$ (d, $\left.J_{\mathrm{P}-\mathrm{C}} 2.6 \mathrm{~Hz}\right), 133.78$ (d, $\left.J_{\mathrm{P}-\mathrm{C}} 8.1 \mathrm{~Hz}\right), 141.66$ $\left(\mathrm{d}, J_{\mathrm{P}-\mathrm{C}} 10.6 \mathrm{~Hz}\right) .{ }^{31} \mathrm{P} \mathrm{NMR}\left(\mathrm{CDCl}_{3}, 121.5 \mathrm{MHz}\right): \delta 32.13(\mathrm{~m})$.

Minor: ${ }^{1} \mathrm{H} \mathrm{NMR}\left(\mathrm{CDCl}_{3}, 300 \mathrm{MHz}\right): \delta$ 0.07-1.24 (bm, $\left.\mathrm{BH}_{3}, 3 \mathrm{H}\right), 1.04\left(\mathrm{~d}, J_{\mathrm{P}-\mathrm{H}} 13.7 \mathrm{~Hz}, t-\mathrm{Bu}, 9 \mathrm{H}\right)$, $1.44(\mathrm{~s}, 3 \mathrm{H}), 1.69(\mathrm{bs}, \mathrm{OH}, 1 \mathrm{H}), 2.64-2.76(\mathrm{~m}, 1 \mathrm{H}), 2.82-2.96(\mathrm{~m}, 1 \mathrm{H}), 5.52-5.83(\mathrm{~m}, 2 \mathrm{H}), 7.01-$

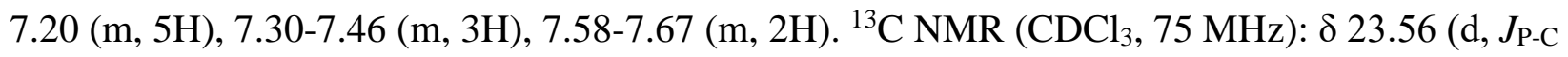
$33.1 \mathrm{~Hz}), 25.49$ (d, $\left.J_{\mathrm{P}-\mathrm{C}} 2.0 \mathrm{~Hz}\right), 29.20,74.22\left(\mathrm{~d}, J_{\mathrm{P}-\mathrm{C}} 1.7 \mathrm{~Hz}\right), 119.14\left(\mathrm{~d}, J_{\mathrm{P}-\mathrm{C}} 4.6 \mathrm{~Hz}\right), 125.09$, 126.82, 128.05, 128.19 (d, $\left.J_{\mathrm{P}-\mathrm{C}} 9.8 \mathrm{~Hz}\right), 131.13$ (d, $\left.J_{\mathrm{P}-\mathrm{C}} 2.6 \mathrm{~Hz}\right), 133.73$ (d, $\left.J_{\mathrm{P}-\mathrm{C}} 8.1 \mathrm{~Hz}\right), 141.97$ $\left(\mathrm{d}, J_{\mathrm{P}-\mathrm{C}} 10.3 \mathrm{~Hz}\right) .{ }^{31} \mathrm{P} \mathrm{NMR}\left(\mathrm{CDCl}_{3}, 121.5 \mathrm{MHz}\right): \delta 32.13(\mathrm{~m})$. Elem. anal. for $\mathrm{C}_{21} \mathrm{H}_{30} \mathrm{BOP}$ : calc. C 74.13, H 8.89; found C 74.01, H 8.70.

Diphenyl-(4-hydroxy-4-phenylpent-1-en-1-yl)phosphine-borane (8bl). Yield 67\% from 0.058 g (0.24 mmol of $\mathbf{1 b}$, purified with hexane/EtOAc (6:1), colorless oil. ${ }^{1} \mathrm{H} \mathrm{NMR}\left(\mathrm{CDCl}_{3}, 300\right.$ $\mathrm{MHz}): \delta$ 0.24-1.43 (bm, $\left.\mathrm{BH}_{3}, 3 \mathrm{H}\right), 1.53$ (s, 3H), 1.77 (bs, OH, 1H), 2.65-2.71 (m, 2H), 5.88-6.01 $(\mathrm{m}, 1 \mathrm{H}), 6.26-6.44(\mathrm{~m}, 1 \mathrm{H}), 7.15-7.41(\mathrm{~m}, \mathrm{Ph}, 15 \mathrm{H}) .{ }^{13} \mathrm{C} \mathrm{NMR}\left(\mathrm{CDCl}_{3}, 75 \mathrm{MHz}\right): \delta 29.88,49.69$ $\left(\mathrm{d}, J_{\mathrm{P}-\mathrm{C}} 14.7 \mathrm{~Hz}\right), 74.32\left(\mathrm{~d}, J_{\mathrm{P}-\mathrm{C}} 0.9 \mathrm{~Hz}\right), 122.55\left(\mathrm{~d}, J_{\mathrm{P}-\mathrm{C}} 56.9 \mathrm{~Hz}\right), 124.71,126.87,128.30,128.62$ $\left(\mathrm{d}, J_{\mathrm{P}-\mathrm{C}} 10.4 \mathrm{~Hz}\right), 128.64\left(\mathrm{~d}, J_{\mathrm{P}-\mathrm{C}} 10.1 \mathrm{~Hz}\right), 129.54\left(\mathrm{~d}, J_{\mathrm{P}-\mathrm{C}} 58.9 \mathrm{~Hz}\right), 130.93$ (d, $\left.J_{\mathrm{P}-\mathrm{C}} 2.6 \mathrm{~Hz}\right)$, $130.97\left(\mathrm{~d}, J_{\mathrm{P}-\mathrm{C}} 2.3 \mathrm{~Hz}\right), 132.35\left(\mathrm{~d}, J_{\mathrm{P}-\mathrm{C}} 9.5 \mathrm{~Hz}\right), 132.37$ (d, $\left.J_{\mathrm{P}-\mathrm{C}} 9.8 \mathrm{~Hz}\right), 146.61,147.79$ (d, $J_{\mathrm{P}-\mathrm{C}} 6.9$ $\mathrm{Hz}) .{ }^{31} \mathrm{P}$ NMR $\left(\mathrm{CDCl}_{3}, 121.5 \mathrm{MHz}\right): \delta 13.66(\mathrm{~m})$. Elem. anal. for $\mathrm{C}_{23} \mathrm{H}_{26} \mathrm{BOP}$ : calc. C 76.68, $\mathrm{H}$ 7.27; found $\mathrm{C} 76.50, \mathrm{H} 7.10$.

5-(1'-Hydroxy-1'-phenylethyl)-2-phospholene-borane (12cl). Yield 76\% from $0.050 \mathrm{~g}(0.28$ mmol) of 1c, purified with hexane/EtOAc (6:1), colorless oil. ${ }^{1} \mathrm{H}$ NMR $\left(\mathrm{CDCl}_{3}, 300 \mathrm{MHz}\right): \delta$ 0.23-1.29 (bm, BH $3,3 \mathrm{H}), 1.63(\mathrm{~s}, 3 \mathrm{H}), 2.01-2.08(\mathrm{~m}, 2 \mathrm{H}), 3.56-3.64(\mathrm{~m}, 1 \mathrm{H}), 6.06-6.22(\mathrm{~m}, 1 \mathrm{H})$, 6.75-6.92 (m, 1H), 7.24-7.44 (m, 8H), 7.50-7.61 (m, 2H). ${ }^{13} \mathrm{C} \mathrm{NMR}\left(\mathrm{CDCl}_{3}, 75 \mathrm{MHz}\right): \delta 25.86$ $\left(\mathrm{d}, J_{\mathrm{P}-\mathrm{C}} 39.4 \mathrm{~Hz}\right), 27.69,58.74,75.46\left(\mathrm{~d}, J_{\mathrm{P}-\mathrm{C}} 5.2 \mathrm{~Hz}\right), 125.05,125.25$ (d, $\left.J_{\mathrm{P}-\mathrm{C}} 49.1 \mathrm{~Hz}\right), 127.19$, 128.34, $128.70\left(\mathrm{~d}, J_{\mathrm{P}-\mathrm{C}} 9.8 \mathrm{~Hz}\right), 130.01\left(\mathrm{~d}, J_{\mathrm{P}-\mathrm{C}} 46.8 \mathrm{~Hz}\right), 131.33$ (d, $\left.J_{\mathrm{P}-\mathrm{C}} 2.6 \mathrm{~Hz}\right), 131.55$ (d, $J_{\mathrm{P}-\mathrm{C}}$ $9.8 \mathrm{~Hz}), 150.42\left(\mathrm{~d}, J_{\mathrm{P}-\mathrm{C}} 7.2 \mathrm{~Hz}\right) .{ }^{31} \mathrm{P} \mathrm{NMR}\left(\mathrm{CDCl}_{3}, 121.5 \mathrm{MHz}\right): \delta 45.15(\mathrm{~m})$. Elem. anal. for $\mathrm{C}_{18} \mathrm{H}_{22} \mathrm{BOP}$ : calc. C 73.00, H 7.49; found C 73.11, H 7.54. 
t-Butyl-(4-hydroxy-4-methyl-hexa-1,5-dien-1-yl)phenylphosphine-borane (8am). Mixture of two diastereoisomers, dr 58:42, yield $84 \%$ from $0.074 \mathrm{~g}(0.33 \mathrm{mmol})$ of 1a, purified with hexane/EtOAc (6:1), colorless oil.

Major: ${ }^{1} \mathrm{H} \mathrm{NMR}\left(\mathrm{CDCl}_{3}, 300 \mathrm{MHz}\right): \delta$ 0.12-1.42 (bm, BH $\left.3,3 \mathrm{H}\right), 1.07\left(\mathrm{~d}, J_{\mathrm{P}-\mathrm{H}} 14.1 \mathrm{~Hz}, t-\mathrm{Bu}, 9 \mathrm{H}\right)$, 1.29 (s, 3H, Me), 2.50-2.54 (m, 2H), $5.05\left(\mathrm{dd}, J_{\mathrm{H}-\mathrm{H}} 1.0 \mathrm{~Hz}, J_{\mathrm{H}-\mathrm{H}} 10.7 \mathrm{~Hz}, 1 \mathrm{H}\right), 5.20$ (dd, $J_{\mathrm{H}-\mathrm{H}} 1.0$ $\left.\mathrm{Hz}, J_{\mathrm{H}-\mathrm{H}} 17.3 \mathrm{~Hz}, 1 \mathrm{H}\right), 5.91\left(\mathrm{dd}, J_{\mathrm{H}-\mathrm{H}} 10.7 \mathrm{~Hz}, J_{\mathrm{H}-\mathrm{H}} 17.3 \mathrm{~Hz}, 1 \mathrm{H}\right), 6.29-6.34(\mathrm{~m}, 1 \mathrm{H}), 6.71-6.88$ $(\mathrm{m}, 1 \mathrm{H}), 7.35-7.49(\mathrm{~m}, 3 \mathrm{H}), 7.65-7.75(\mathrm{~m}, 2 \mathrm{H}) .{ }^{31} \mathrm{P} \mathrm{NMR}\left(\mathrm{CDCl}_{3}, 121.5 \mathrm{MHz}\right): \delta 25.44(\mathrm{~m})$.

Minor: ${ }^{1} \mathrm{H}$ NMR $\left(\mathrm{CDCl}_{3}, 300 \mathrm{MHz}\right)$ : $\delta$ 012-1.42 (bm, BH $\left.3,3 \mathrm{H}\right), 1.07$ (d, $\left.J_{\mathrm{P}-\mathrm{H}} 14.1 \mathrm{~Hz}, t-\mathrm{Bu}, 9 \mathrm{H}\right)$, 1.29 (s, 3H, Me), 2.50-2.54 (m, 2H), $5.04\left(\mathrm{dd}, J_{\mathrm{H}-\mathrm{H}} 1.0 \mathrm{~Hz}, J_{\mathrm{H}-\mathrm{H}} 10.8 \mathrm{~Hz}, 1 \mathrm{H}\right), 5.18$ (dd, $J_{\mathrm{H}-\mathrm{H}} 1.0$ $\left.\mathrm{Hz}, J_{\mathrm{H}-\mathrm{H}} 17.4 \mathrm{~Hz}, 1 \mathrm{H}\right), 5.90\left(\mathrm{dd}, J_{\mathrm{H}-\mathrm{H}} 10.8 \mathrm{~Hz}, J_{\mathrm{H}-\mathrm{H}} 17.4 \mathrm{~Hz}, 1 \mathrm{H}\right), 6.24-6.28(\mathrm{~m}, 1 \mathrm{H}), 6.71-6.88$ $(\mathrm{m}, 1 \mathrm{H}), 7.35-7.49(\mathrm{~m}, 3 \mathrm{H}), 7.65-7.75(\mathrm{~m}, 2 \mathrm{H}) .{ }^{31} \mathrm{P} \mathrm{NMR}\left(\mathrm{CDCl}_{3}, 121.5 \mathrm{MHz}\right): \delta 25.92(\mathrm{~m})$. Elem. anal. for $\mathrm{C}_{17} \mathrm{H}_{28}$ BOP: calc. C 70.36, H 9.73; found C 70.69, H 9.49.

(4-Hydroxy-4-methyl-hexa-1,5-dien-1-yl)diphenylphosphine-borane (8bm) and (4-hydroxy4-methyl-hexa-1,5-dien-3-yl)diphenylphosphine-borane (10bm). Product ratio 45:55, yield 91\% from $0.084 \mathrm{~g}(0.35 \mathrm{mmol})$ of $\mathbf{1 b}$, purified with hexane/EtOAc (6/1), colorless oil.

Compound 8bm. ${ }^{1} \mathrm{H}$ NMR $\left(\mathrm{CDCl}_{3}, 300 \mathrm{MHz}\right): \delta$ 0.12-1.25 (bm, $\left.\mathrm{BH}_{3}, 3 \mathrm{H}\right), 1.23$ (s, 3H), 2.46$2.52(\mathrm{~m}, 2 \mathrm{H}), 5.01\left(\mathrm{dd}, J_{\mathrm{H}-\mathrm{H}} 1.4 \mathrm{~Hz}, J_{\mathrm{H}-\mathrm{H}} 10.8 \mathrm{~Hz}, 1 \mathrm{H}\right), 5.18\left(\mathrm{dd}, J_{\mathrm{H}-\mathrm{H}} 1.4 \mathrm{~Hz}, J_{\mathrm{H}-\mathrm{H}} 17.3 \mathrm{~Hz}, 1 \mathrm{H}\right)$, $5.90\left(\mathrm{dd}, J_{\mathrm{H}-\mathrm{H}} 1.4 \mathrm{~Hz}, J_{\mathrm{H}-\mathrm{H}} 10.8 \mathrm{~Hz}, 1 \mathrm{H}\right), 6.12-6.29(\mathrm{~m}, 1 \mathrm{H}), 6.55-6.71(\mathrm{~m}, 1 \mathrm{H}), 7.34-7.51$ (m, $6 \mathrm{H}), 7.55-7.71(\mathrm{~m}, 4 \mathrm{H}) .{ }^{31} \mathrm{P}$ NMR $\left(\mathrm{CDCl}_{3}, 121.5 \mathrm{MHz}\right): \delta 13.21(\mathrm{~m})$.

Compound 12bm (mixture of diastereomers). ${ }^{1} \mathrm{H}$ NMR $\left(\mathrm{CDCl}_{3}, 300 \mathrm{MHz}\right): \delta$ 0.12-1.25 (bm, $\left.\mathrm{BH}_{3}, 6 \mathrm{H}\right), 1.25(\mathrm{~s}, 3 \mathrm{H}), 1.26(\mathrm{~s}, 3 \mathrm{H}), 2.99-3.08(\mathrm{~m}, 1 \mathrm{H}), 3.22-3.33(\mathrm{~m}, 1 \mathrm{H}), 4.79-4.86(\mathrm{~m}, 1 \mathrm{H})$, 4.81-4.88 (m, 1H), 5.03-5.05 (m, 1H), 5.06-5.08 (m, 1H), 5.56-5.71 (m, 4H), 6.08-6.14 (m, 1H), 6.14-6.20 (m, 1H), 6.51-6.73 (m, 2H), 7.34-7.51 (m, 12H), 7.55-7.71 (m, 8H). ${ }^{31} \mathrm{P} \mathrm{NMR}\left(\mathrm{CDCl}_{3}\right.$, $121.5 \mathrm{MHz}): \delta 21.39$ and $21.81(\mathrm{~m})$.

$\boldsymbol{t}$-Butyl-(4-hydroxy-4-methyl-oct-1-en-1-yl)phenylphosphine-borane (8an). Yield 81\% from $0.047 \mathrm{~g}(0.21 \mathrm{mmol})$ of 1a, purified with hexane/EtOAc $(6 / 1)$, colorless oil. ${ }^{1} \mathrm{H} \mathrm{NMR}\left(\mathrm{CDCl}_{3}\right.$, $300 \mathrm{MHz}): \delta$ 0.17-1.30 (bm, BH $3,3 \mathrm{H}), 0.89\left(\mathrm{t}, J_{\mathrm{H}-\mathrm{H}} 6.95 \mathrm{~Hz}, 3 \mathrm{H}\right), 1.10\left(\mathrm{~d}, J_{\mathrm{P}-\mathrm{H}} 13.7 \mathrm{~Hz}, t-\mathrm{Bu}\right.$, $9 \mathrm{H}), 1.19(\mathrm{~s}, 3 \mathrm{H}), 1.27-1.35(\mathrm{~m}, 4 \mathrm{H}), 1.42-1.49(\mathrm{~m}, 2 \mathrm{H}), 1.53(\mathrm{bs}, 1 \mathrm{H}), 2.47$ (bd, $\left.J_{\mathrm{H}-\mathrm{H}} 7.31 \mathrm{~Hz}\right)$, 6.27-6.39 (m, 1H), 6.79-6.97 (m, 1H), 7.38-7.51 (m, 3H), 7.69-7.77 (m, 2H). ${ }^{31} \mathrm{P} \mathrm{NMR}\left(\mathrm{CDCl}_{3}\right.$, 121.5 MHz): 25.40 ppm (m). Elem. anal. for $\mathrm{C}_{19} \mathrm{H}_{34} \mathrm{BOP}$ : calc. C 71.26, H 10.70; found C 71.35, H 10.92 .

4-(t-Butylphenylboranatophosphinyl)buten-3-oic acid (8ao). Yield 76\% from $0.062 \mathrm{~g}(0.29$ mmol) of 1a, purified with hexane/EtOAc (1/1), colorless oil. ${ }^{1} \mathrm{H}$ NMR $\left(\mathrm{CDCl}_{3}, 300 \mathrm{MHz}\right): \delta$ 0.08-1.29 (bm, BH $3,3 \mathrm{H}), 1.03$ (d, $\left.J_{\mathrm{P}-\mathrm{H}} 14.4 \mathrm{~Hz}, t-\mathrm{Bu}, 9 \mathrm{H}\right), 3.28-3.33(\mathrm{~m}, 2 \mathrm{H}), 6.33-6.43(\mathrm{~m}, 1 \mathrm{H})$, 6.73-6.90 (m, 1H), 7.32-7.43 (m, 3H), 7.62-7.70 (m, 2H), 9.84 (bs, 1H). ${ }^{13} \mathrm{C} \mathrm{NMR}\left(\mathrm{CDCl}_{3}, 75\right.$ MHz): 25.37 (d, $\left.J_{\mathrm{P}-\mathrm{C}} 2.9 \mathrm{~Hz}\right), 29.42$ (d, $\left.J_{\mathrm{P}-\mathrm{C}} 34.8 \mathrm{~Hz}\right), 47.72\left(\mathrm{~d}, J_{\mathrm{P}-\mathrm{C}} 14.4 \mathrm{~Hz}\right), 117.78$ (d, $J_{\mathrm{P}-\mathrm{C}} 54.0$ $\mathrm{Hz}), 128.18$ (d, J $\left.J_{\mathrm{P}-\mathrm{C}} 9.5 \mathrm{~Hz}\right), 128.19$ (d, $\left.J_{\mathrm{P}-\mathrm{C}} 51.7 \mathrm{~Hz}\right), 130.86$ (d, J J-C $\left.2.6 \mathrm{~Hz}\right), 133.05$ (d, $J_{\mathrm{P}-\mathrm{C}} 7.8$ $\mathrm{Hz}), 150.42$ (d, $\left.J_{\mathrm{P}-\mathrm{C}} 8.62 \mathrm{~Hz}\right), 207.12 .{ }^{31} \mathrm{P} \mathrm{NMR}\left(\mathrm{CDCl}_{3}, 121.5 \mathrm{MHz}\right): 26.40 \mathrm{ppm}(\mathrm{m})$. Elem. anal. for $\mathrm{C}_{14} \mathrm{H}_{22} \mathrm{BO}_{2} \mathrm{P}$ : calc. C 63.67, $\mathrm{H}$ 8.40; found $\mathrm{C}$ 63.50, H 8.36. 
4-(Diphenylboranatophosphinyl)buten-3-oic acid (8bo). Yield 92\% from $0.125 \mathrm{~g}(0.52 \mathrm{mmol})$ of 1b, purified with EtOAc as eluent), white solid, m.p. 145-147 ${ }^{\circ} \mathrm{C} .{ }^{1} \mathrm{H} \mathrm{NMR}\left(\mathrm{CDCl}_{3}, 300\right.$ $\mathrm{MHz}$ ): $\delta$ 0.25-1.58 (bm, $\left.\mathrm{BH}_{3}, 3 \mathrm{H}\right), 3.20-3.27$ (m, 2H), 3.37 (bs, 1H), 6.17-6.30 (m, 1H), 6.48-

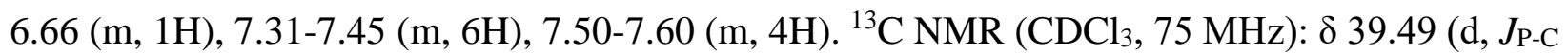
$15.8 \mathrm{~Hz}$ ), 122.69 (d, $\left.J_{\mathrm{P}-\mathrm{C}} 57.2 \mathrm{~Hz}\right), 128.70$ (d, $\left.J_{\mathrm{P}-\mathrm{C}} 10.1 \mathrm{~Hz}\right), 129.09$ (d, $\left.J_{\mathrm{P}-\mathrm{C}} 59.5 \mathrm{~Hz}\right), 131.13$ (d, $\left.J_{\mathrm{P}-\mathrm{C}} 2.3 \mathrm{~Hz}\right), 132.36\left(\mathrm{~d}, J_{\mathrm{P}-\mathrm{C}} 9.8 \mathrm{~Hz}\right), 143.53$ (d, $\left.J_{\mathrm{P}-\mathrm{C}} 8.3 \mathrm{~Hz}\right), 172.10 .{ }^{31} \mathrm{P} \mathrm{NMR}\left(\mathrm{CDCl}_{3}, 121.5\right.$ $\mathrm{MHz}$ ): 13.84 (m). Elem. anal. for $\mathrm{C}_{16} \mathrm{H}_{18} \mathrm{BO}_{2} \mathrm{P}$ : calc. C 67.64, H 6.39; found C 67.70, H 6.50.

2-(t-Butylphenylboranatophosphinyl)pent-2-eno-1,5-dicarboxylic acid dimethyl ester (13ar). Yield $40 \%$ from $0.051 \mathrm{~g}(0.23 \mathrm{mmol})$ of 1a, purified with hexane/EtOAc $(6 / 1)$ as eluent, colorless oil. ${ }^{1} \mathrm{H} \mathrm{NMR}\left(\mathrm{CDCl}_{3}, 300 \mathrm{MHz}\right): \delta$ 0.08-1.23 (bm, 3H, BH $), 1.06\left(\mathrm{~d}, J_{\mathrm{P}-\mathrm{H}} 14.4 \mathrm{~Hz}, t\right.$ $\mathrm{Bu}, 9 \mathrm{H}), 2.99-3.11(\mathrm{~m}, 1 \mathrm{H}), 3.34-3.48(\mathrm{~m}, 1 \mathrm{H}), 3.65(\mathrm{~s}, 3 \mathrm{H}), 3.79(\mathrm{~s}, 3 \mathrm{H}), 7.01-7.10(\mathrm{~m}, 1 \mathrm{H})$, 7.34-7.48 (m, 3H), 7.61-7.69 (m, 2H). ${ }^{13} \mathrm{C} \mathrm{NMR}\left(\mathrm{CDCl}_{3}, 75 \mathrm{MHz}\right): \delta 25.28\left(\mathrm{~d}, J_{\mathrm{P}-\mathrm{C}} 2.6 \mathrm{~Hz}\right)$, $29.84\left(\mathrm{~d}, J_{\mathrm{P}-\mathrm{C}} 30.5 \mathrm{~Hz}\right), 30.91,52.39,52.47,118.24$ (d, $\left.J_{\mathrm{P}-\mathrm{C}} 50.6 \mathrm{~Hz}\right), 128.41$ (d, $\left.J_{\mathrm{P}-\mathrm{C}} 9.5 \mathrm{~Hz}\right)$, $129.45\left(\mathrm{~d}, J_{\mathrm{P}-\mathrm{C}} 52.0 \mathrm{~Hz}\right), 131.62\left(\mathrm{~d}, J_{\mathrm{P}-\mathrm{C}} 2.9 \mathrm{~Hz}\right), 133.47$ (d, $\left.J_{\mathrm{P}-\mathrm{C}} 8.6 \mathrm{~Hz}\right), 150.27$ (d, $\left.J_{\mathrm{P}-\mathrm{C}} 8.9 \mathrm{~Hz}\right)$, 206.93, 210.14. ${ }^{31} \mathrm{P} \mathrm{NMR}\left(\mathrm{CDCl}_{3}, 121.5 \mathrm{MHz}\right): \delta 34.60 \mathrm{ppm}(\mathrm{m})$. Elem. anal. for $\mathrm{C}_{17} \mathrm{H}_{26} \mathrm{BO}_{4} \mathrm{P}$ : calc. C 60.74, H 7.80; found C 60.47, H 7.62.

2-(Diphenylboranatophosphinyl)pent-2-eno-1,5-dicarboxylic acid dimethyl ester (13br). Yield $86 \%$ from $0.106 \mathrm{~g}(0.44 \mathrm{mmol})$ of 1a, purified with hexane/EtOAc $(6 / 1)$ as eluent, colorless oil. ${ }^{1} \mathrm{H} \mathrm{NMR}\left(\mathrm{CDCl}_{3}, 300 \mathrm{MHz}\right): \delta$ 0.32-1.58 (bm, $\left.\mathrm{BH}_{3}, 3 \mathrm{H}\right), 3.36-3.45(\mathrm{~m}, 2 \mathrm{H}), 3.63$ $(\mathrm{s}, 3 \mathrm{H}), 3.67(\mathrm{~s}, 3 \mathrm{H}), 6.07-7.06(\mathrm{~m}, 1 \mathrm{H}), 7.34-7.48(\mathrm{~m}, 6 \mathrm{H}), 7.56-7.64(\mathrm{~m}, 4 \mathrm{H}) .{ }^{13} \mathrm{C} \mathrm{NMR}$ $\left(\mathrm{CDCl}_{3}, 75 \mathrm{MHz}\right): \delta 52.29,52.51,127.66\left(\mathrm{~d}, J_{\mathrm{P}-\mathrm{C}} 54.3 \mathrm{~Hz}\right), 128.93\left(\mathrm{~d}, J_{\mathrm{P}-\mathrm{C}} 10.1 \mathrm{~Hz}\right), 128.66(\mathrm{~d}$, $\left.J_{\mathrm{P}-\mathrm{C}} 52.3 \mathrm{~Hz} \mathrm{Ph}\right), 131.67\left(\mathrm{~d}, J_{\mathrm{P}-\mathrm{C}} 2.6 \mathrm{~Hz}\right), 132.28\left(\mathrm{~d}, J_{\mathrm{P}-\mathrm{C}} 9.2 \mathrm{~Hz}\right), 1541.30\left(\mathrm{~d}, J_{\mathrm{P}-\mathrm{C}} 2.9 \mathrm{~Hz}\right), 163.76$ $\left(\mathrm{d}, J_{\mathrm{P}-\mathrm{C}} 2.0 \mathrm{~Hz}\right), 134.76\left(\mathrm{~d}, J_{\mathrm{P}-\mathrm{C}} 2.3 \mathrm{~Hz}\right) .{ }^{31} \mathrm{P} \mathrm{NMR}\left(\mathrm{CDCl}_{3}, 121.5 \mathrm{MHz}\right): 18.49$ (m). Elem. anal. for $\mathrm{C}_{19} \mathrm{H}_{22} \mathrm{BO}_{4} \mathrm{P}$ : calc. C 64.07, H 6.23; found C 64.30, H 6.40.

$\boldsymbol{t}$-Butyl-1-(4-hydroxy-4-(p-nitrophenyl)but-1-en-1-yl)phenylphosphine-borane

(8ap).

Mixture of two diastereoisomers, yield $18 \%$ based on ${ }^{1} \mathrm{H}$ NMR spectra of the isolated fraction from $0.040 \mathrm{~g}(0.18 \mathrm{mmol})$ of 1a, purified with hexane/EtOAc $(6 / 1) .{ }^{1} \mathrm{H} \mathrm{NMR}\left(\mathrm{CDCl}_{3}, 300\right.$ $\mathrm{MHz}): \delta$ 0.22-1.16 (bm, BH $3,3 \mathrm{H}), 0.95\left(\mathrm{~d}, J_{\mathrm{P}-\mathrm{H}} 14.1 \mathrm{~Hz}, t-\mathrm{Bu}, 9 \mathrm{H}\right)$ and $0.97\left(\mathrm{~d}, J_{\mathrm{P}-\mathrm{H}} 14.1 \mathrm{~Hz}, t\right.$ $\mathrm{Bu}, 9 \mathrm{H}), 2.23$ (bs, OH, 1H), 2.55-2.78 (m, 2H), 4.83-5.02 (m, 1H), 6.07-6.24 (m, 1H), 6.62-6.86 $(\mathrm{m}, 1 \mathrm{H}), 7.26-7.49(\mathrm{~m}, 5 \mathrm{H}), 7.96-8.06(\mathrm{~m}, 2 \mathrm{H}), 8.08-8.15(\mathrm{~m}, 2 \mathrm{H}) .{ }^{31} \mathrm{P} \mathrm{NMR}\left(\mathrm{CDCl}_{3}, 121.5\right.$ $\mathrm{MHz}): \delta 25.57$ (bm).

p-Nitrobenzyl alcohol (14p). Yield 26\%, based on ${ }^{1} \mathrm{H}$ NMR spectra of the isolated fraction. ${ }^{1} \mathrm{H}$ NMR $\left(\mathrm{CDCl}_{3}, 300 \mathrm{MHz}\right): \delta 2.25$ (bs, OH, 1H), 4,74 (s, 2H), 7.42-7.47 (m, 2H), 8.10-8.14 (m, $2 \mathrm{H})$.

t-Butyl-1-(4-hydroxy-4-(o-nitrophenyl)but-1-en-1-yl)phenylphosphine-borane

(8aq). Mixture of two diastereoisomers, yield $36 \%$ based on ${ }^{1} \mathrm{H}$ NMR spectra of the isolated fraction from $0.050 \mathrm{~g}(0.23 \mathrm{mmol})$ of 1a, purified with hexane/EtOAc $(6 / 1) .{ }^{1} \mathrm{H} \mathrm{NMR}\left(\mathrm{CDCl}_{3}, 300\right.$ $\mathrm{MHz}): \delta$ 0.06-1.20 (bm, BH $3,3 \mathrm{H}), 0.97\left(\mathrm{~d}, J_{\mathrm{P}-\mathrm{H}} 14.1 \mathrm{~Hz}, t-\mathrm{Bu}, 9 \mathrm{H}\right)$ and $1.00\left(\mathrm{~d}, J_{\mathrm{P}-\mathrm{H}} 13.8 \mathrm{~Hz}, t\right.$ $\mathrm{Bu}, 9 \mathrm{H}), 2.46$ (bs, OH, 1H), 2.48-2.73 (m, 2H), 5.10-5.20 (m, 1H), 6.20-6.35 (m, 1H), 6.78-6.96 
$(\mathrm{m}, 1 \mathrm{H}), 7.26-7.47(\mathrm{~m}, 5 \mathrm{H}), 7.50-7.75(\mathrm{~m}, 3 \mathrm{H}), 7.98-8.05(\mathrm{~m}, 1 \mathrm{H}) .{ }^{31} \mathrm{P} \mathrm{NMR}\left(\mathrm{CDCl}_{3}, 121.5\right.$ $\mathrm{MHz}): \delta 25.57(\mathrm{~m})$.

$\boldsymbol{o}$-Nitrobenzyl alcohol (14q). Yield 29\%, based on ${ }^{1} \mathrm{H}$ NMR spectra of the isolated fraction. ${ }^{1} \mathrm{H}$ NMR $\left(\mathrm{CDCl}_{3}, 300 \mathrm{MHz}\right): \delta 2.46$ (bs, OH, 1H), 4.89 (s, 2H), 7.30-7.44 (m, 1H), 7.50-7.75 (m, $1 \mathrm{H}), 7.77-7.88(\mathrm{~m}, 1 \mathrm{H})$.

\section{Acknowledgements}

Authors are grateful to Dr Duncan Carmichael (École Polytechnique, Palaiseau, France) for his guidance during the preparation of manuscript.

\section{References}

1. (a) King, R. B.; Cloyd, J. C.; Reimann, R. H. J. Org. Chem. 1976, 41, 972. (b) Soulier, E.; Yaouanc, J.-J.; Laurent, P.; des Abbayes, H.; Clément, J.-C. Eur. J. Org. Chem. 2000, 3497. (c) Han, L.-B.; Zhao, C.-Q. J. Org. Chem. 2005, 70, 10121. (d) Bunlaksananusorn, T.; Knochel, P. J. Org. Chem. 2004, 69, 4595. (e) Zhang, Y.; Pullarkat, S. A.; Li, Y.; Leung, P.H. Inorg. Chem. 2009, 48, 5535.

2. Griffiths, D. V.; Groombridge, H. J.; Mahoney, P. M.; Swetnam, S. P.; Walton, G.; York, D. C. Tetrahedron 2005, 61, 4595.

3. (a) Schuman, M.; Trevitt, M.; Redd, A.; Gouverneur, V. Angew. Chem. Int. Ed. 2000, 39, 2491. (b) Demchuk, O. M.; Pietrusiewicz, K. M.; Michrowska, A.; Grela, K. Org. Lett. 2003, 5, 3217. (c) Slinn, C. A.; Redgrave, A. J.; Hind, L. S.; Edlin, C.; Nolan, S. P.; Gouverneur, V. Org. Biomol. Chem. 2003, 1, 3820. (d) Bisaro, F.; Gouverneur, V. Tetrahedron 2005, 61, 2395. (e) Vinokurov, N.; Michrowska, A.; Szmigielska, A.; Drzazga, Z.; Wojciuk, G.; Demchuk, O. M.; Grela, K.; Pietrusiewicz, K. M.; Butenschön, H. Adv. Synth. Catal. 2006, 348, 931. (f) Harvey, J. S.; Malcolmson, S. J.; Dunne, K. S.; Meek, S. J.; Thompson, A. L.; Schrock, R. R.; Hoveyda, A. H.; Gouverneur, V. Angew. Chem. Int. Ed. 2009, 48, 762.

4. (a) Affandi, S.; Nelson, J. H.; Fisher, J. Inorg. Chem. 1989, 28, 4536. (b) Aw, B.-H.; Hor, T. S. A.; Selvaratnam, S.; Mok, K. F.; White, A. J. P.; Williams, D. J.; Rees, N. H.; McFarlane, W.; Leung, P.-H. Inorg. Chem. 1997, 36, 2138. (c) Yeo, W.-C.; Vittal, J. J.; Koh, L. L.; Tan, G.-K.; Leung, P.-H. Organomet. 2004, 23, 3474. (d) Ma, M.; Pullarkat, S. A.; Yuan, M.; Zhang, N.; Li, Y.; Leung, P.-H. Organomet. 2009, 28, 4886.

5. (a) Crimmins, M. T.; King, B. W. J. Am. Chem. Soc. 1998, 120, 9084. (b) Murakami, N.; Sugimoto, M.; Nakajima, T.; Kawanishi, M.; Tsutsui, Y.; Kobayashi, M. Bioorg. Med. Chem. 2000, 8, 2651. 
6. Yamano, Y.; Sato, Y.; Watanabe, Y.; Namikawa, K.; Miki, W.; Ito, M. J. Chem. Soc., Perkin Trans. 1 2001, 1862.

7. Bhatt, U.; Christmann, M.; Quitschalle, M.; Claus, E.; Kalesse, M. J. Org. Chem. 2001, 66, 1885. (b) Williams, D. R.; Ihle, D. C.; Plummer, S. V. Org. Lett. 2001, 3, 1383.

8. Marumoto, S.; Kogen, H.; Naruto, S. J. Org. Chem. 1998, 63, 2068.

9. Waterson, A. G.; Kruger, A. W.; Meyers, A. I. Tetrahedron Lett. 2001, 42, 4305.

10. Tortosa, M.; Yakelis, N. A.; Roush, W. A. J. Org. Chem. 2008, 73, 9657.

11. (a) Phillips, A. M. M. M.; Modro, T. A. Phosphorus, Sulfur, Silicon 1991, 55, 41. (b) Gil, J. M.; Hah, J. H.; Park, K. Y.; Oh, D. Y. Synth. Commun. 2000, 30, 789. (c) Al-Badri, H.; About-Jaudet, E.; Collignon, N. Tetrahedron Lett. 1995, 36, 393. (d) Probst, M. F.; Modro, A. M.; Modro, T. A. Can. J. Chem. 1997, 75, 1131. (e) Christov, V. C. Phosphorus, Sulfur, Silicon 1998, 132, 73. (f) Yuan, C.; Yao, J.; Li, S. Phosphorus, Sulfur, Silicon 1990, 53, 21. (g) Al-Badri, H.; About-Jaudet, E.; Collignon, N. Synthesis 1994, 1072.

12. (a) Muller, E. L.; Modro, A. M.; Modro, T. A. Bull. Soc. Chim. Fr. 1994, 131, 959. (b) Piffl, M.; Weston, J.; Anders, E. Eur. J. Org. Chem. 2000, 2851.

13. (a) Phillips, A. M. M. M.; Modro, T. A. J. Chem. Soc. Perkin Trans. 1 1991, 1875. (b) Gerber, K. P.; Modro, T. A.; Muller, E. L.; Phillips, A. M. Phosphorus, Sulfur, Silicon 1993, 75, 19. (c) Tanaka, K.; Ohta, Y.; Fuji, K. J. Org. Chem. 1995, 60, 8036.

14. (a) Kirilov, M.; Petrova, J.; Zdravkova, Z. Phosphorus, Sulfur 1985, 21, 301. (b) Hanessian, S.; Andreotti, D.; Gomtsyan, A. J. Am. Chem. Soc. 1995, 117, 10393. (c) Denmark, S. E.; Kim, J.-H. J. Org. Chem. 1995, 60, 7535. (d) Maleki, M.; Miller, J. A. Tetrahedron Lett. 1981, 22, 3789. (e) Grayson, J. I.; Warren, S.; Zaslona, A.T J. Chem. Soc. Perkin Trans. 1 1987, 967. (f) Binns, M. R.; Haynes, R. K.; Katsifis, A. G.; Schober, P. A.; Vonwiller, S. C. J. Am. Chem. Soc. 1988, 110, 5411. (g) Ergüden, J.-K.; Schaumann, E. Synthesis 1996, 707.

15. For reviews, see: (a) Brunel, J. M.; Faure, B.; Maffei, M. Coord. Chem. Rev. 1998, 178-180, 665. (b) Ohff, H.; Holz, J.; Quirmbach, M.; Börner, A. Synthesis, 1998, 1391. (c) Carboni, B.; Monnier, L. Tetrahedron 1999, 55, 1197.

16. (a) Pakulski, Z.; Kwiatosz, R.; Pietrusiewicz, K. M. Tetrahedron Lett. 2003, 44, 5469. (b) Pakulski, Z.; Kwiatosz, R.; Pietrusiewicz, K. M. Tetrahedron 2005, 61, 1481.

17. Pakulski, Z.; Demchuk, O. M.; Frelek, J.; Luboradzki, R.; Pietrusiewicz, K. M. Eur. J. Org. Chem. 2004, 3913. 